\title{
HIGH FREQUENCY PHOTOACOUSTIC CHARACTERIZATION OF SINGLE CELLS
}

\author{
by \\ Eric Strohm \\ M.Sc. Ryerson University, Toronto, Canada, 2009 \\ B.Sc. McMaster University, Hamilton, Canada, 1999 \\ A dissertation \\ presented to Ryerson University \\ in partial fulfillment of the \\ requirements for the degree of \\ Doctor of Philosophy \\ in the Program of \\ Biomedical Physics
}

Toronto, Ontario, Canada, 2014

(C) Eric Strohm, 2014 


\section{Author's Declaration}

I hereby declare that I am the sole author of this dissertation. This is a true copy of the dissertation, including any required final revisions, as accepted by my examiners.

I authorize Ryerson University to lend this dissertation to other institutions or individuals for the purpose of scholarly research

I further authorize Ryerson University to reproduce this dissertation by photocopying or by other means, in total or in part, at the request of other institutions or individuals for the purpose of scholarly research.

I understand that my dissertation may be made electronically available to the public.

Eric Strohm 


\section{Abstract}

High frequency photoacoustic characterization of single cells. Doctor of Philosophy 2014, Eric Strohm, Biomedical Physics, Ryerson University.

This dissertation presents the first photoacoustic study of single cells using ultra-high frequencies (UHF, over $100 \mathrm{MHz}$ ). At these frequencies, unique features occur in the photoacoustic signal spectrum which depend on the cell size, morphology and structure. A finite element model (FEM) was developed to simulate the photoacoustic signals from ideal spherical droplets containing a perfluorocarbon liquid and optically absorbing nanoparticles.

The model was applied to droplets in suspension and on a boundary to examine how the photoacoustic spectrum varies with droplet size and configuration, and compared to measurements using a $375 \mathrm{MHz}$ transducer. Good agreement in the spectral features between the measured values and the FEM and analytical solution were observed. For the droplet on a boundary, additional spectral features were observed there were correctly predicted by the FEM, but not the analytical solution. The FEM could be applied to situations where the analytical model cannot be used, such as the asymmetric shape of red blood cells (RBCs). Measurements of single RBCs were then compared to the FEM. The frequency location of the spectral minima shifted to higher frequencies as the $\mathrm{RBC}$ rotated from a vertical to horizontal orientation. The spectral minima shifted to lower frequencies as the RBC swelled 
from the normal biconcave shape to a spherical morphology. Healthy RBCs were differentiated from spherocytes, echinocytes and swollen RBCs using changes in the photoacoustic spectrum $(\mathrm{p}<0.001)$. These results suggest that the photoacoustic spectrum can be used to classify RBCs according to their shape and pathology. Classification of cells using the photoacoustic spectral features was applied to measurements of blood cells and circulating tumor cells (CTCs) such as melanoma and acute myeloid leukemia (AML) cells. Measurements of 89 cells showed that variations in the spectrum and signal amplitude could be used to identify and differentiate melanoma and AML cells from RBCs, thus identifying foreign cells in the bloodstream. This dissertation investigates how UHF photoacoustics can be used to identify and classify cells and particles in a sample using their photoacoustic spectra, with the end goal of using these methods to identify cell pathology and detect CTCs clinically. 


\section{Acknowledgements}

First and foremost, I would like to express my sincere gratitude to my supervisor Dr. Michael Kolios for providing guidance, support and patience throughout my studies. His encouragement to explore the unknown helped shape both the focus of this dissertation, and my abilities as a researcher.

I'm grateful for the extensive guidance from members of my supervisory and examination committees, Drs. Carl Kumaradas, Raffi Karshafian and Yuan Xu. Their helpful discussions clarified the overall direction of this dissertation.

The many members of the Kolios lab, past and present made the past few years a fun and enjoyable experience. The revolving door of the graduate research lab is a constant source of new faces, abilities and ideas that have directly and indirectly contributed to this work. In particular, I would like to acknowledge Arthur Worthington for his excellent device building (and repair) abilities, Elizabeth Berndl for extensive biological related support and Eno Hysi for entertaining conversations and assistance with blood-related experiments.

Last but not least, this dissertation could not have been completed without the support of my family: my parents and sister for encouraging my scientific curiosity; my wife for her support and confidence in my abilities, particularly during busy or difficult times; and my inlaws for providing extensive support in countless ways over the past few years. 


\section{Table of Contents}

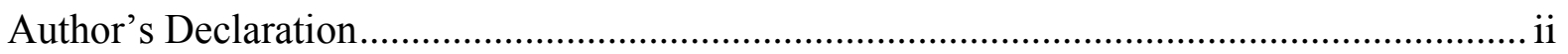

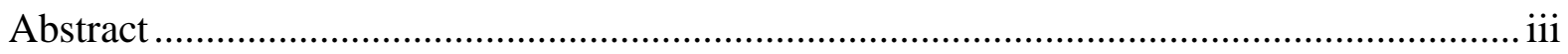

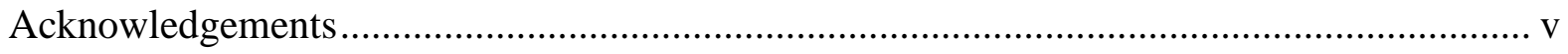

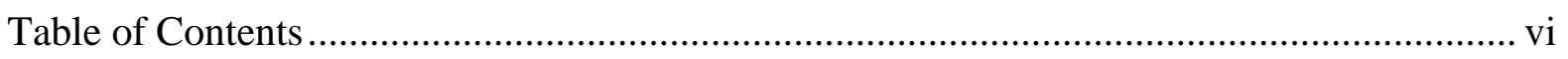

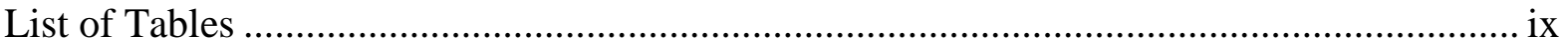

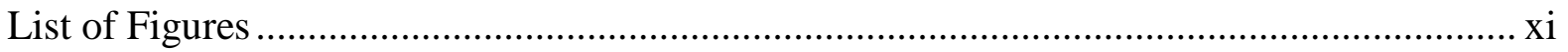

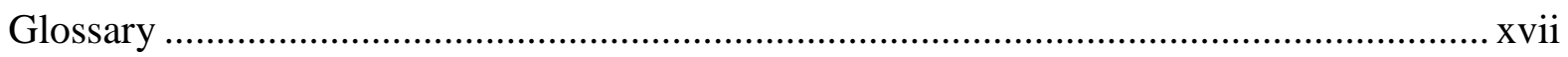

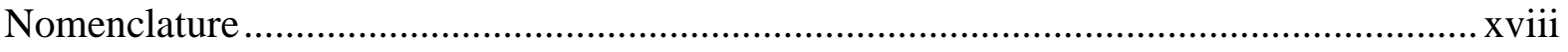

Chapter 1 Introduction ............................................................................................... 1

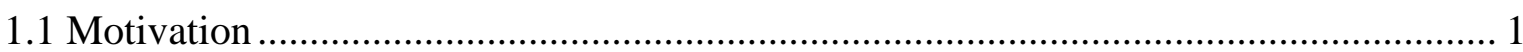

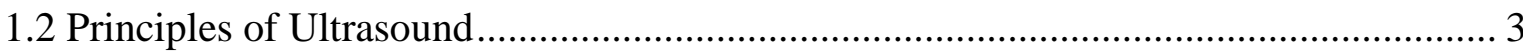

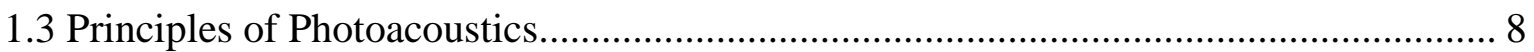

1.4 Probing single cells with ultrasound and photoacoustics........................................... 15

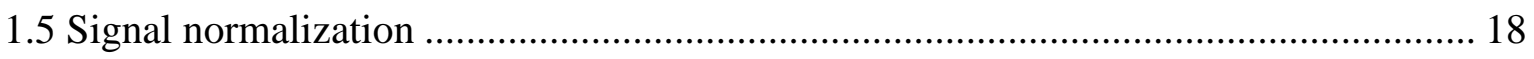

1.6 Modeling ultrasound and photoacoustic interactions................................................. 20

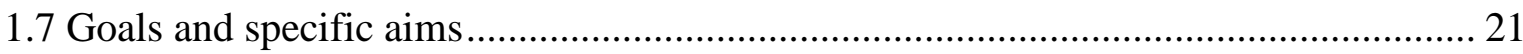

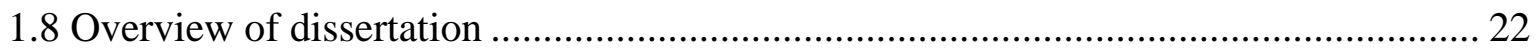

Chapter 2 A Photoacoustic finite element model to describe spectral features of micron-sized

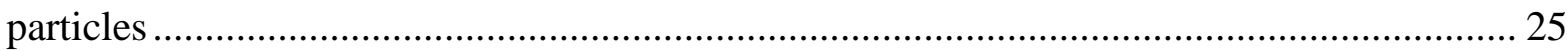

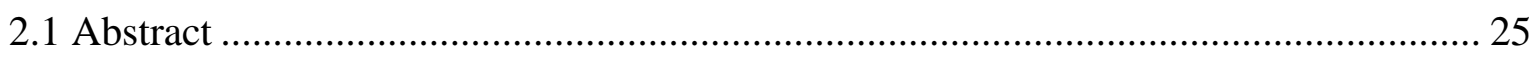

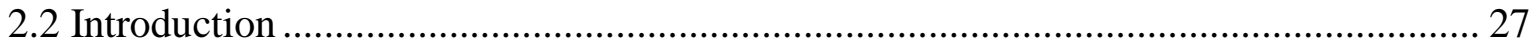

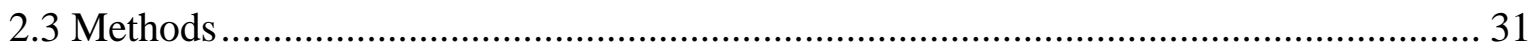

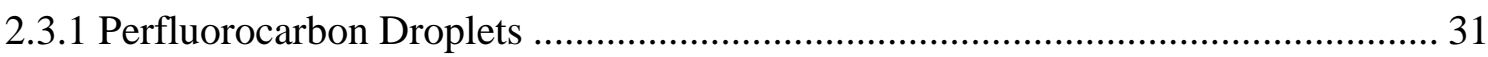

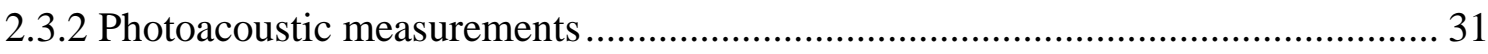




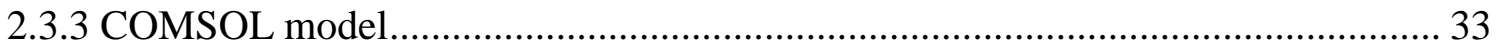

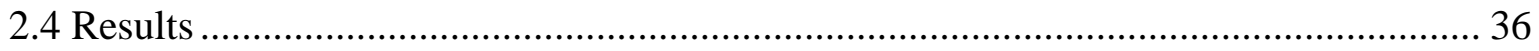

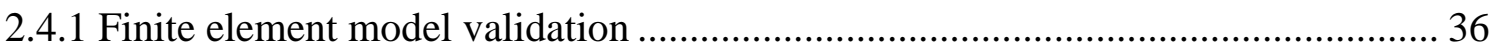

2.4.2 Model and experiment comparison …………...................................................... 37

2.4.3 Droplet surrounded by an absorbing ring ........................................................ 41

2.4.4 Visualization of the propagating signal ............................................................ 42

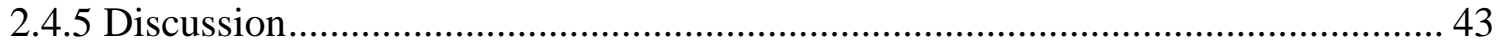

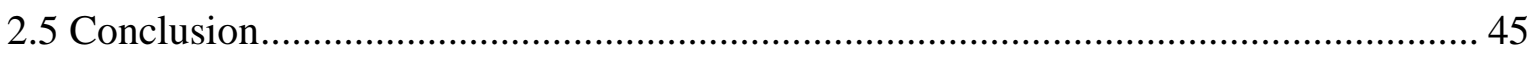

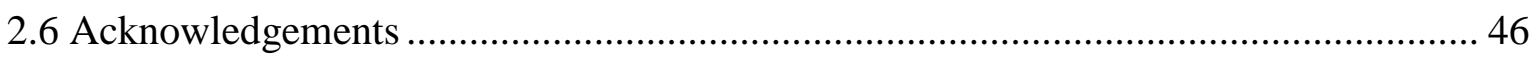

Chapter 3 Acoustic and photoacoustic characterization of micron-sized perfluorocarbon

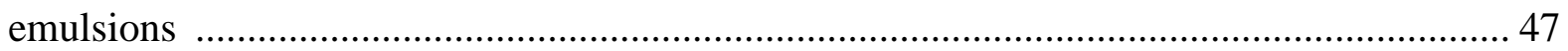

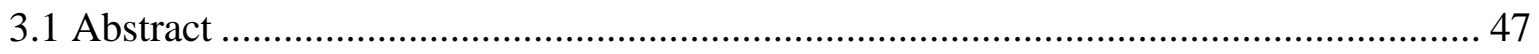

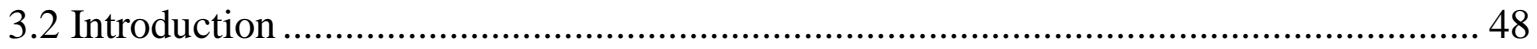

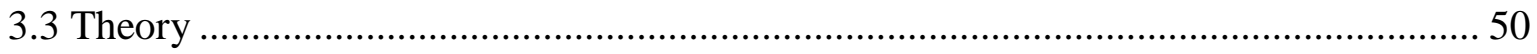

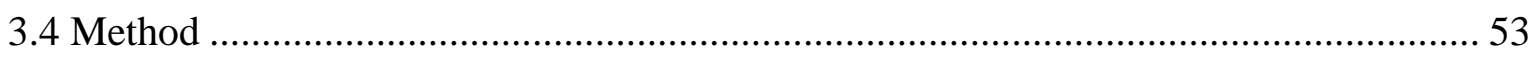

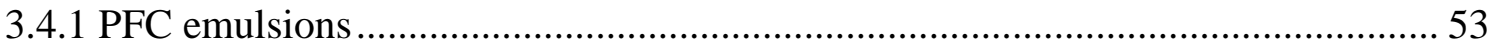

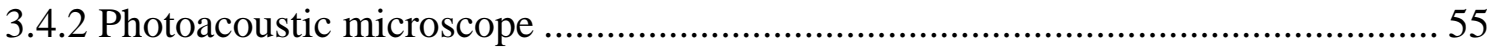

3.4.3 Acoustic and photoacoustic measurements ......................................................... 58

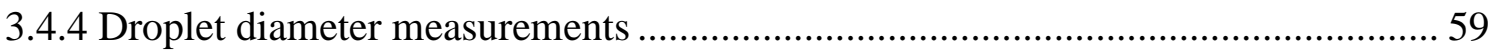

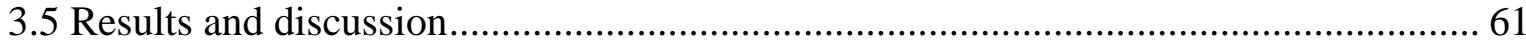

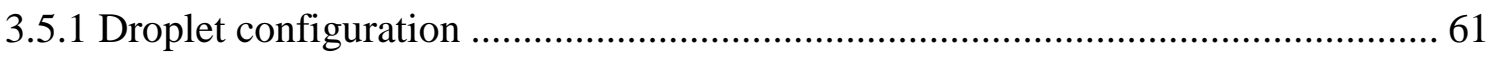

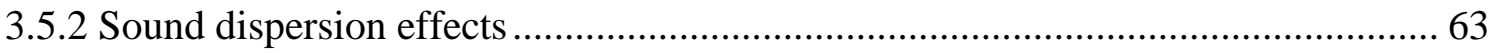

3.5.3 Droplet diameter measurements ............................................................................ 64

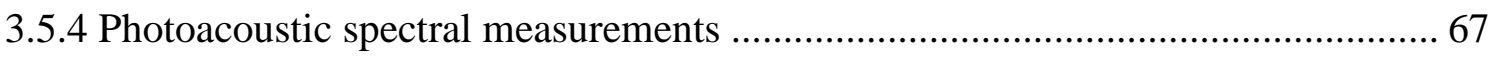

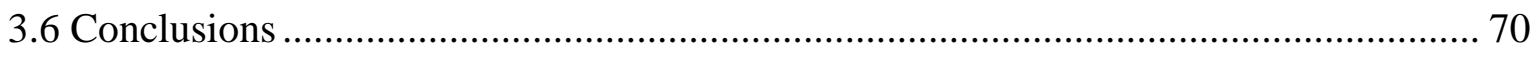

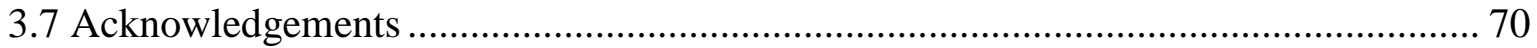

Chapter 4 Probing red blood cell morphology using high frequency photoacoustics ........... 72

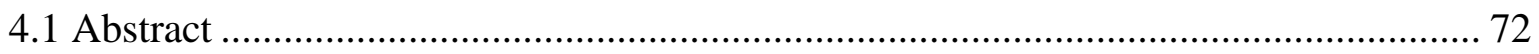

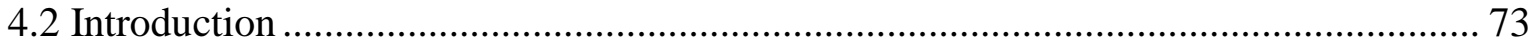

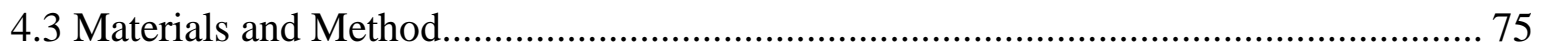




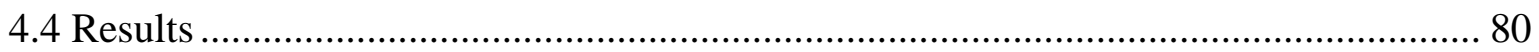

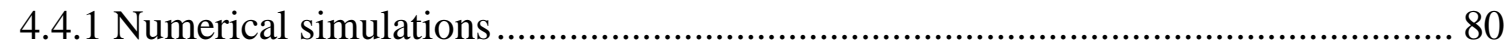

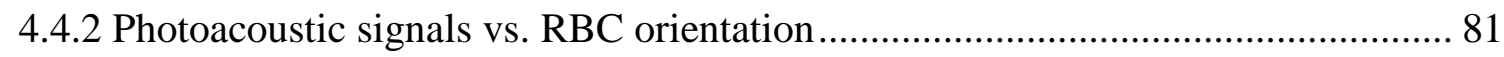

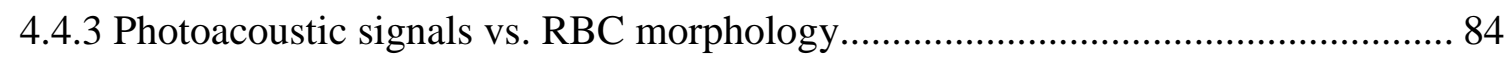

4.4.4 Quantitative Spectral Analysis ............................................................................ 89

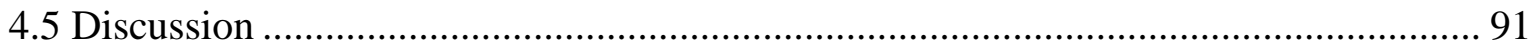

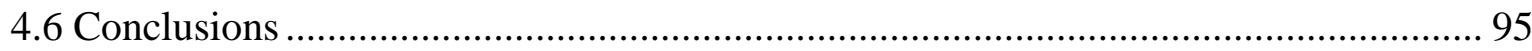

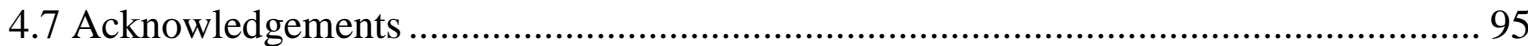

Chapter 5 Circulating tumor cell detection using high frequency photoacoustics .................. 97

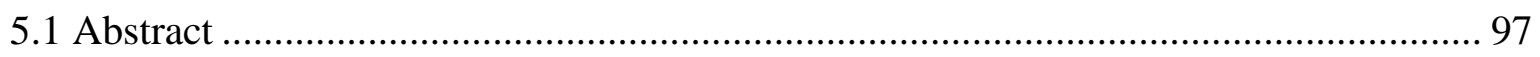

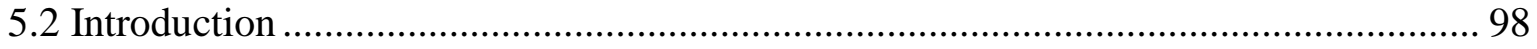

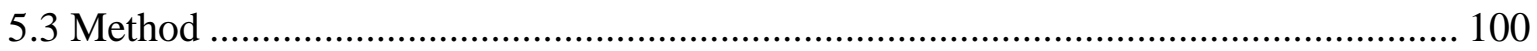

5.3.1 Cell Preparation ............................................................................................ 100

5.3.2 Photoacoustic Microscope ……………………............................................... 100

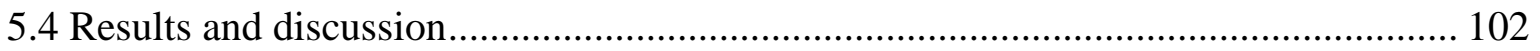

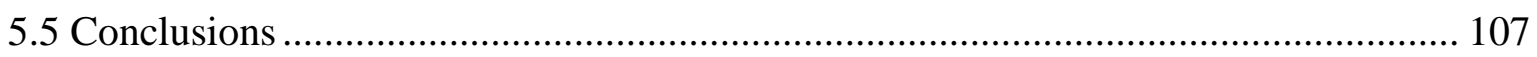

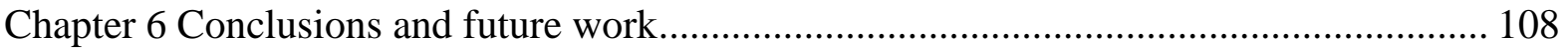

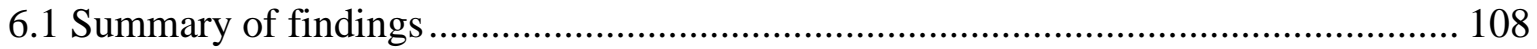

6.1.1 Photoacoustic numerical method development (chapter 2)................................. 108

6.1.2 Model validation for ideal photoacoustic particles (chapter 3) ........................... 109

6.1.3 Application towards irregularly shaped RBCs (chapter 4) ................................... 111

6.1.4 Cell differentiation and identification (chapter 5) ............................................. 112

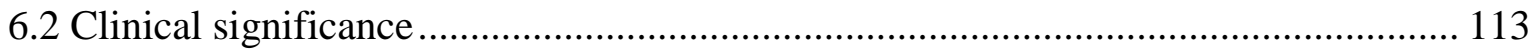

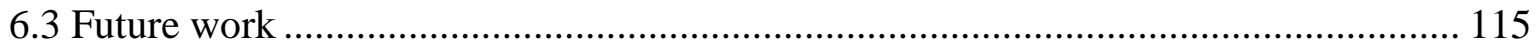

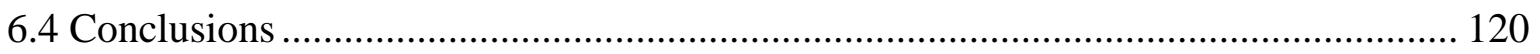

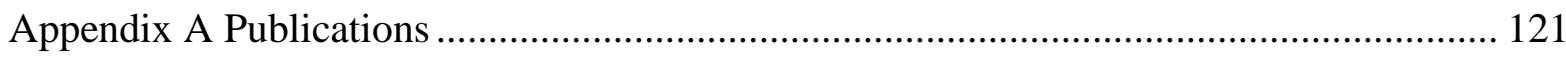

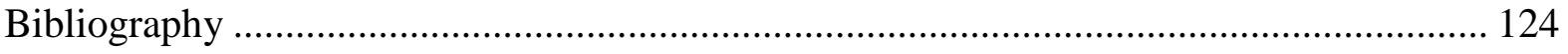




\section{List of Tables}

Table 2-1. Modeling parameters used in the finite element method models. 36

Table 2-2: Spectral minima frequencies of the five droplets in Figure 2.6. The spectral minima associated with the glass substrate and not observed using the analytical model are coloured red. The last column is the average standard deviation between the

frequency minima of the FEM and measured spectrum. 41

Table 3-1: The diameters of 18 droplets calculated using the photoacoustic time domain signal (equation 3.3), the ultrasound time domain signal (equation 4), spectral comparison between model and experiment (equation 3.2) and optical microscopy. The uncertainties in the photoacoustic and ultrasound methods were calculated based on the accuracy of determining the locations of the signal peaks in figure 1, and the model uncertainty from the diameter variations in fitting the experiment to theory. The optical method uncertainty was large due to the difficulty in determining the droplet edges, and the limited number of pixels used when acquiring the droplet image.

Table 4-1: Quantitative parameters for RBCs in PBS with varying osmolalities. The spectral amplitude and spectral slope were calculated between 275-300 MHz in Fig. 5 for the RBCs immersed in PBS with osmolalities ranging from 294 to $84 \mathrm{mmol} / \mathrm{kg}$. A statistically significant difference in the spectral amplitude and spectral slope was found between each change in osmolality from 294 to $118 \mathrm{mmol} / \mathrm{kg}(\mathrm{p}<0.001)$ but not between 118 and $84 \mathrm{mmol} / \mathrm{kg}$. At these two osmolalities, the RBCs were spherical and the spectral shapes are similar.

Table 4-2: Quantitative parameters of healthy and abnormal RBCs. The spectral amplitude and spectral slope were calculated between 275-300 MHz in Fig. 6 for fresh RBCs in DMEM, echinocytes in PBS, and then the same echinocytes deposited in DMEM. The echinocytes were formed by immersing the fresh RBCs in PBS for 24 hours, which then 
regained their normal biconcave shape after immersion in DMEM. A statistically significant difference in the spectral amplitude and slope was observed between fresh RBCs and the echinocytes ( $\mathrm{p}<0.001$ ). No statistically significant difference was observed between fresh RBCs and the depleted RBCs restored in DMEM ( $p<0.05)$,

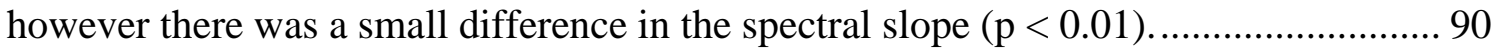

Table 5-1: A summary of the diameter (measured optically), spectral spacing $\Delta f$ and normalized signal amplitude for the different cell types. 107 


\section{List of Figures}

Figure 1.1: Change in ultrasound resolution with frequency for transducers with different fnumbers.

Figure 1.2: Ultrasound attenuation (left) and backscatter (right) images of a 1.4 diameter MCF7 spheroid measured using an $80 \mathrm{MHz}$ transducer. In the attenuation image, the dark areas indicate areas of strong attenuation, while in the backscatter image, the light areas indicate areas of strongest backscatter.

Figure 1.3: Optical (left), ultrasound backscatter (middle) and ultrasound attenuation (right) images of a single MCF7 cell using $375 \mathrm{MHz}$ (top) and $1000 \mathrm{MHz}$ (bottom). The scale bar is $15 \mu \mathrm{m}$.

Figure 1.4: Illustration of how photoacoustic and ultrasound waves are generated from a single spherical particle. In photoacoustics, the particle is irradiated by a laser, absorbs the energy and emits a pressure wave with ultrasonic frequencies. In ultrasound, the particle is insonified by incident pressure waves, which then reflect from the particle.... 9

Figure 1.5: The theoretical photoacoustic time domain signal of a $20 \mu \mathrm{m}$ diameter liquid sphere with a sound speed of $1560 \mathrm{~m} / \mathrm{s}$ and a density of $1050 \mathrm{~kg} / \mathrm{m}^{3}$. 10

Figure 1.6: Photoacoustic images of a fixed B16-F1 melanoma cell imaged with progressively higher transducer frequencies. The laser spot size remained the same at approximately $5 \mu \mathrm{m}$; therefore the imaging resolution is a function of the transducer, not the laser spot. (A) Optical image with fluorescence inset, (B) Photoacoustic image using $200 \mathrm{MHz}$, (C) $375 \mathrm{MHz}$ and (D) $1200 \mathrm{MHz}$. The scale bar is $30 \mu \mathrm{m}$. (Figure adapted from [80])

Figure 1.7: The theoretical photoacoustic signal of the $20 \mu \mathrm{m}$ spherical particle shown in Figure 1.5 (black) when filtered with a 100-500 MHz bandpass filter (red), simulating the limited bandwidth of a typical UHF transducer. 
Figure 1.8: The time domain signal (left) and spectrum (right) of a $375 \mathrm{MHz}$ transducer used for photoacoustics (blue) and ultrasound (red), along with the theoretical spectrum for a $200 \mathrm{~nm}$ thick gold film (black). The $-6 \mathrm{~dB}$ bandwidth for ultrasound was $165 \mathrm{MHz}$ (285$450 \mathrm{MHz}$ ), but the same transducer used for photoacoustics had a $215 \mathrm{MHz}$ bandwidth (245-460 MHz).

Figure 2.1: Diagram showing the two droplet configurations measured. The transducer positioned above the droplet recorded the photoacoustic signals from the droplet when irradiated by a $1064 \mathrm{~nm}$ laser from below. The photoacoustic signals from a droplet with a soft boundary (droplet on an agar surface, left) and a hard boundary (droplet directly on a glass substrate) were measured.

Figure 2.2: COMSOL model geometries used in this study. (A) a $3.1 \mu \mathrm{m}$ droplet in a water suspension, (B) a droplet on glass (diameters ranged from 2.8 to $3.6 \mu \mathrm{m}$ ) and (C) a 3.1 $\mu \mathrm{m}$ droplet in a water suspension but with an optically absorbing $250 \mathrm{~nm}$ shell surrounding a non-absorbing core. In (A) and (B), the pressure inside the droplet was set to unity, and the surround water the pressure was set to zero. In (C), the pressure of the ring was set to unity, and the core was set to zero. The signal was recorded directly above the droplet.

Figure 2.3: Theoretical waveform and spectrum $3.1 \mu \mathrm{m}$ droplet (top) and a $5.0 \mu \mathrm{m}$ droplet (bottom) suspended in water obtained from the analytical solution (equation 2-2, solid black line) and the FEM solution (dotted red line). The signals were normalized to maximum amplitude for comparison. Good agreement in both the time and frequency domains were observed, verifying the FEM methodology.

Figure 2.4: A comparison of the FEM solution of a $3.1 \mu \mathrm{m}$ droplet on glass (red) and the analytical solution of the same droplet in suspension (black). The signals were normalized to maximum amplitude for comparison. Small changes in the time domain signal result in extra spectral minima.

Figure 2.5: A representative measured photoacoustic signal from (A) $3.1 \mu \mathrm{m}$ droplet in suspension, and (B) a different $3.1 \mu \mathrm{m}$ droplet on a glass substrate.

Figure 2.6: The photoacoustic spectrum from (A) a $3.1 \mu \mathrm{m}$ droplet on an agar surface (B-E) a droplet on glass with diameters $2.8,3.1,3.6$ and $3.6 \mu \mathrm{m}$ in diameter, respectively. The measured signals are blue, the analytical solution is black and the FEM solution is red. 
Good agreement in the location of the spectral minima measured and modeled solutions were observed for the droplet on an agar surface. For the droplet on a glass substrate, good agreement was observed between measured and the FEM solution only. The spectral region outside the transducer $-12 \mathrm{~dB}$ bandwidth of approximately $190-540 \mathrm{MHz}$ is noise and has been shaded to reduce attention to this area.

Figure 2.7: The theoretical photoacoustic spectrum from a $3.1 \mu \mathrm{m}$ droplet in suspension with an optically absorbing ring $250 \mathrm{~nm}$ thick surrounded a non-absorbing center (dotted blue line) compared to the analytical solution of a homogeneously absorbing liquid sphere in black 42

Figure 3.1: (A) The acoustic microscope is an inverted optical microscope where the laser has been focused through the pack port onto the sample. The mirror reflects $500-650 \mathrm{~nm}$ (the laser wavelengths) towards the sample, but allows other wavelengths to pass for optical viewing. The transducer measures the signals from above the sample. (B) A close-up view of a measurement of a single droplet. The laser is focused onto the sample using the optical objective with the transducer positioned directly above the droplet.... 57 Figure 3.2: (A) The theoretical photoacoustic time domain signal from a $5 \mu \mathrm{m}$ droplet using equation 3.2 (black dotted line), and the same signal after applying a Hamming window and 100-500 MHz bandpass filter (red solid line). (B) The measured ultrasound echoes from a $7.2 \mu \mathrm{m}$ PFH droplet embedded in gelatin. The signals labeled $t_{1}$ and $t_{2}$ were used to determine the droplet diameter using equation 3.3 (photoacoustic, left) and equation 3.4 (ultrasound, right).

Figure 3.3: Transmission electron microscopy image (A) and optical absorption coefficient (B) of the silica coated gold NP PFH solution. The gold NPs were approximately $20 \mathrm{~nm}$ in diameter with a $5 \mathrm{~nm}$ thick silica coating. The peak optical absorption was between $530-540 \mathrm{~nm}$. The scale bar is $20 \mathrm{~nm}$.

Figure 3.4: The effects of dispersion on the photoacoustic model showing the time domain solution (A) and frequency domain solution (B) for a $5 \mu \mathrm{m}$ droplet using equation 3.1 (constant sound speed, black solid line) and equation 3.2 (sound speed increasing from 480 to $486 \mathrm{~m} / \mathrm{s}$, red dashed line). A Hamming window and 300-1400 MHz bandpass filter were used for both solutions. Good agreement was found up to $300 \mathrm{MHz}$, but 
discrepancies in the location of the spectral minima and maxima were observed over 500 $\mathrm{MHz}$

Figure 3.5: The measured photoacoustic signal (red dashed line) compared to the model (black solid line, equation 3.2) for a $5.7 \mu \mathrm{m}$ droplet measured at $375 \mathrm{MHz}$ (A, B), a 2.45 $\mu \mathrm{m}$ droplet measured at $750 \mathrm{MHz}(\mathrm{C}, \mathrm{D})$ and a $6.95 \mu \mathrm{m}$ droplet measured at $750 \mathrm{MHz}$ (E, F). A bandpass filter of 100-500 MHz was used for the $375 \mathrm{MHz}$ measurement, and a bandpass filter of $300-1000 \mathrm{MHz}$ was used for the $750 \mathrm{MHz}$ measurements. A Hamming window was applied to all measurements. Good agreement between model and experiment were found for the waveforms $(\mathrm{A}, \mathrm{C}, \mathrm{E})$ and in the location of the spectral minima and maxima $(\mathrm{B}, \mathrm{D}, \mathrm{F})$.

Figure 4.1: The photoacoustic microscope. (a) The SASAM photoacoustic microscope. The sample is positioned between the transducer and optical objective. (b) RBCs after deposition into the sample holder. Most RBCs align horizontally on the substrate, however a small number align vertically as indicated by the arrow. The scale bar is 20 $\mu \mathrm{m}$. (c) A schematic showing the sample positioning relative to the transducer and focused laser.

Figure 4.2: The finite element model. The finite element model developed to describe the photoacoustic waves generated by micron sized particles. The RBC was positioned at the center, and transducers recorded the emitted photoacoustic wave around the RBC. 79

Figure 4.3: Numerical simulations results using the finite element model. (a) The photoacoustic spectrum as the transducer position was varied from $0^{\circ}$ (vertical $\mathrm{RBC}$ ) to $90^{\circ}$ (horizontal RBC) for a $7.82 \mu \mathrm{m}$ diameter RBC. (b) The photoacoustic spectrum calculated from a vertical RBC as the diameter was varied from $6.80 \mu \mathrm{m}$ to $8.80 \mu \mathrm{m} \ldots 82$

Figure 4.4: Photoacoustic measurements of single RBCs. (a) The time domain signal as measured from a single $7.8 \mu \mathrm{m}$ diameter RBC when aligned vertically (blue) and horizontally (red) relative to the transducer measured in PBS at isotonic conditions. (b) The photoacoustic power spectrum of the RBCs shown in Figure 4.3A when aligned vertically (blue) and horizontally (red) compared to the numerical simulations for their respective orientation (dashed black line). (c) The photoacoustic spectrum of a $5.8 \mu \mathrm{m}$ diameter RBC induced into a spherical shape by immersion in a hypotonic solution of 84 $\mathrm{mmol} / \mathrm{kg}$ (solid red line) compared to numerical simulations of a spherical morphology 
(dashed black line). A schematic of the RBC shape and orientation is shown inset within the figure. The transducer bandwidth is from about 150 to $550 \mathrm{MHz}$ as indicated by the dotted arrow. The signal amplitude has been normalized to match theory. 83

Figure 4.5: Photoacoustic spectral variations with RBC morphology. (a) The photoacoustic spectrum from an average of 21 RBCs measured in PBS with decreasing osmolality. The error bars indicate the standard deviation. As the osmolality decreases, the RBCs swell resulting in a gradual decrease of the photoacoustic spectral amplitude. Optical images confirm the change in morphology, however it is difficult to quantify the change. The scale bar is $10 \mu \mathrm{m}$. (b, c) The photoacoustic spectrum of each individual RBC measurement (red), and the average (black) of all 21 measurements at $294 \mathrm{mmol} / \mathrm{kg}$ (b) and $147 \mathrm{mmol} / \mathrm{kg}(\mathrm{c})$ 87

Figure 4.6: Photoacoustic spectrum of healthy RBCs and echinocytes. (a) The photoacoustic spectrum of RBCs in DMEM cell culture medium (red), left in PBS for 24 hours resulting in the formation of echinocytes (black), then the echinocytes deposited in cell culture medium to restore the normal biconcave shape (blue). Images of the RBCs at the three stages are shown inset. The photoacoustic spectrum of the echinocytes is very different than the normal RBCs. The spectrum between the RBCs in fresh medium (red) and ATP-depleted RBCs in medium (blue) are similar, showing that the echinocytes can be restored to their normal biconcave shape. The scale bar is $10 \mu \mathrm{m}$. (b, c) The photoacoustic spectrum of each individual RBC measurement (red) and the average (black) of all 21 measurements of echinocytes in PBS (b) and then in medium (c). A much larger variation between spectra is observed for the echinocytes compared to normal or swollen RBCs. 88

Figure 5.1: The photoacoustic microscope. (A) A picture of the sample holder, with the transducer positioned above the sample and the optics to view the sample and focus the laser are positioned below the sample. (B) A schematic showing the operation of the photoacoustic microscope. The laser is focused onto the cell using an optical objective. The resulting photoacoustic waves are then recorded by the transducer. 102

Figure 5.2: The time domain signals recorded from (A) RBCs in a horizontal (green) and vertical (blue) orientation, and (B) melanoma cells (red) and AML cells (black). 105 
Figure 5.3: Typical photoacoustic power spectra from cells. The spectrum of melanoma cells (A, red) and AML cells (B, black) compared to RBCs measured in a horizontal (green) and vertical (blue) orientation. The area outside of the transducer bandwidth has been grayed. 106

Figure 5.4: The measured photoacoustic signal (normalized to laser energy) vs. the photoacoustic spectral spacing $\Delta f$. The location of the cells in this graph can be used to identify them. 106

Figure 6.1: Basic schematic of the flow device showing the particle flow through the PAUS target area.

Figure 6.2: Simultaneous measurements of a single cell using photoacoustics and ultrasound. (A) Recorded a-scan showing both the photoacoustic and ultrasound signal, and zoomed image of the photoacoustic signal (B) and the ultrasound signal (C) 118

Figure 6.3: The millifluidic device. The top layer (A) is placed on top of the middle layer (B), which has grooves cut into the plastic for particle flow. (C) The layers are glued together to create a 3D structure. Tubes are attached to the nozzles for fluid flow. The size of the bottom structure is $25 \times 75 \mathrm{~mm}$....

Figure 6.4: Flow focusing performance of the microfluidic device at the inlet/mixing area (left) and optical view of the focused stream at the observation region (right). The particles are confined to a $15 \mu \mathrm{m}$ width. The scale bar is $20 \mu \mathrm{m}$. 


\section{Glossary}

$\begin{array}{ll}\text { ADV } & \text { Acoustic droplet vaporization } \\ \text { ATP } & \text { Adenosine triphosphate } \\ \text { CTC } & \text { Circulating tumor cell } \\ \text { dB } & \text { deciBel } \\ \text { DMEM } & \text { Dulbecco's modified essential medium } \\ \text { EPR } & \text { Enhanced permeability and retention effect } \\ \text { FBS } & \text { Fetal bovine serum } \\ \text { FEM } & \text { Finite element model } \\ \text { HIFU } & \text { High intensity focused ultrasound } \\ \text { HF } & \text { High frequency ( 20-80 MHz) } \\ \text { NA } & \text { Numerical aperture } \\ \text { NP } & \text { Nanoparticle } \\ \text { ODV } & \text { Optical droplet vaporization } \\ \text { PA } & \text { Photoacoustic } \\ \text { PAM } & \text { Photoacoustic microscopy } \\ \text { PAT } & \text { Photoacoustic tomography } \\ \text { PbS } & \text { Lead sulphide nanoparticle } \\ \text { PBS } & \text { Phosphate buffered saline } \\ \text { PFC } & \text { Perfluorocarbon (generic compound) } \\ \text { PDMS } & \text { Polydimethylsiloxane } \\ \text { PFH } & \left.\text { Perfluorohexane (C } \text { F F } 14_{14}\right) \\ \text { PFP } & \text { Perfluoropentane }\left(\mathrm{C}_{5} \mathrm{~F}_{12}\right) \\ \text { PMMA } & \text { Poly(methyl methacrylate) } \\ \text { QPA } & \text { Quantitative photoacoustic } \\ \text { QUS } & \text { Quantitative ultrasound } \\ \text { RBC } & \text { Red blood cell (erythrocyte) } \\ \text { PRF } & \text { Pulse repetition frequency } \\ \text { SAM } & \text { Scanning acoustic microscope } \\ \text { SASAM } & \text { Saarland scanning acoustic microscope } \\ \text { US } & \text { Ultrasound } \\ \text { UHF } & \text { Ultra-high frequency (over } 100 \mathrm{MHz}) \\ \text { WBC } & \text { White blood cell (leukocyte) } \\ & \end{array}$




\section{Nomenclature}

$\begin{array}{ll}\mathrm{a} & \text { Radius }(\mathrm{m}) \\ \mathrm{c} & \text { Sound speed }(\mathrm{m} / \mathrm{s}) \\ \mathrm{f} & \text { Ultrasonic frequency }(\mathrm{Hz}) \\ \mathrm{p} & \text { Pressure (time domain) }(\mathrm{Pa}) \\ \mathrm{t} & \text { Time }(\mathrm{s}) \\ \mathrm{C}_{\mathrm{p}} & \text { Specific heat capacity }(\mathrm{J} / \mathrm{kg} / \mathrm{K}) \\ \mathrm{H} & \text { Optical energy }(\mathrm{J}) \\ \mathrm{I}_{0} & \text { Laser intensity }\left(\mathrm{W} / \mathrm{m}^{2}\right) \\ \mathrm{P} & \text { Pressure (frequency domain) } \\ \alpha & \text { Thermal diffusivity }\left(\mathrm{m}^{2} / \mathrm{s}\right) \\ \beta & \text { Thermal expansion coefficient }\left(\mathrm{K}^{-1}\right) \\ \lambda & \text { Ultrasound wavelength }\left(\mathrm{m}^{-1}\right) \\ \mu_{\mathrm{a}} & \text { Absorption coefficient }\left(\mathrm{cm}^{-1}\right) \\ \rho & \text { Density }\left(\mathrm{kg} / \mathrm{m}^{3}\right)\end{array}$




\section{Chapter 1}

\section{Introduction}

\subsection{Motivation}

In 2012, an estimated 1.64 million new cases of cancer occurred in the United States, with 577,000 deaths [1]. Cancer is the second highest cause of death behind heart disease, accounting for $23 \%$ of all deaths [1]. Cancerous tumors are not typically detected until they are large enough to be felt or observed, or cause pain and/or body dysfunction. Detection methods include imaging modalities such as ultrasound, $x$-ray and MRI, minimally invasive procedures such as blood tests, and molecular/genetic testing. Early detection and treatment are crucial for improving the cancer survival rate.

Cancer can directly and indirectly affect the cells in blood. Hematological cancers such as leukemia, lymphoma and myeloma account for $9 \%$ of all cancers and originate in or near the blood [1], while primary tumors originating elsewhere in the body can release metastatic cells called circulating tumor cells (CTCs) that travel through the blood stream. The formation of metastatic CTCs leads to $90 \%$ of all cancer-related deaths [2]. CTCs are common in patients with cancer, but not in healthy people [3]; lower CTC concentrations are 
correlated to a better outcome in cancer patients [4-9]. There is limited information about the relation between numbers and heterogeneity of CTCs and types of cancers, and the lack of a gold standard for quantifying CTCs remains an issue [10].

A screening tool that accurately detects circulating tumor cells would aid in early diagnosis and help decrease mortality rates with early treatment. Current CTC identification methods such as flow cytometry require the addition of expensive external agents such as fluorescent dyes, antibodies or enzymes to identify tumor cells. A method to rapidly screen a sample of blood for CTCs using label-free or inexpensive additives does not exist.

Probing single cells using ultrasound or photoacoustic methods requires ultra-high frequencies (UHF, over $100 \mathrm{MHz}$ ) [11]. At these frequencies, the wavelength is similar to the size of the cell, where the resulting signals are dependent on the size, shape and composition of the cell. Our goal is to understand how these interactions work on the single cell level, and how the signals can be used to identify CTCs.

Quantitative UHF ultrasound methods have been used since the 1970's to extract specific information about individual cells; UHF photoacoustic methods are a relatively new development, with little published literature, particularly for cellular examination. The majority of this dissertation is devoted to exploring quantitative photoacoustic methods using frequencies over $100 \mathrm{MHz}$, and how it can be used to differentiate cells, with the end goal of developing a method that can be used to detect tumor cells in a sample such as blood. 


\subsection{Principles of Ultrasound}

This dissertation focuses primarily on photoacoustic measurements of cells and particles. Photoacoustic waves from micron sized objects, such as cells, are pressure waves with ultrasonic frequencies; the propagation of photoacoustic waves are governed by the physics of ultrasound propagation. This section on ultrasound theory and methods will provide an introduction into photoacoustic wave generation and propagation.

Ultrasound was first developed as a diagnostic imaging tool in 1942 by Dr. Karl Dussik [12], however it wasn't until the 1960's that a wide variety of ultrasound devices become readily available [13]. Due to technological limitations, these early systems were limited to frequencies less than $5 \mathrm{MHz}$. These days, ultrasound imaging devices using frequencies up to $15 \mathrm{MHz}$ are widespread in hospitals and clinics, while research specific devices are available with frequencies over $1000 \mathrm{MHz}$ [14].

The ultrasound imaging resolution increases with frequency; however this comes at a cost of reduced penetration depth. Figure 1.1 shows how the lateral ultrasound resolution increases with frequency [11]. Frequencies around $5 \mathrm{MHz}$ typically have resolutions around $0.5 \mathrm{~mm}$ and usually used for fetal sonograms, while frequencies around 40-60 $\mathrm{MHz}$ have resolutions around 20-30 $\mu \mathrm{m}$ and can be used for small animal imaging, and resolving smaller structures near the body surface such as breast tumours. Resolutions up to $1 \mu \mathrm{m}$ can be obtained by increasing the frequency to $1000 \mathrm{MHz}$ which allows for imaging of single cells; however the penetration depth at such a high frequency is only a few cells at best and cannot be used for clinical purposes. Figure 1.2 shows ultrasound images of a $1.4 \mathrm{~mm}$ 
diameter MCF7 tumor spheroid using $80 \mathrm{MHz}$. Increased attenuation through the central necrotic core is observed, while the backscatter image shows increased backscatter from the core. Nutrients and oxygen cannot diffuse into the central core and the cells die, resulting in higher attenuation and backscatter from these cells in comparison to the surrounding viable region [15].

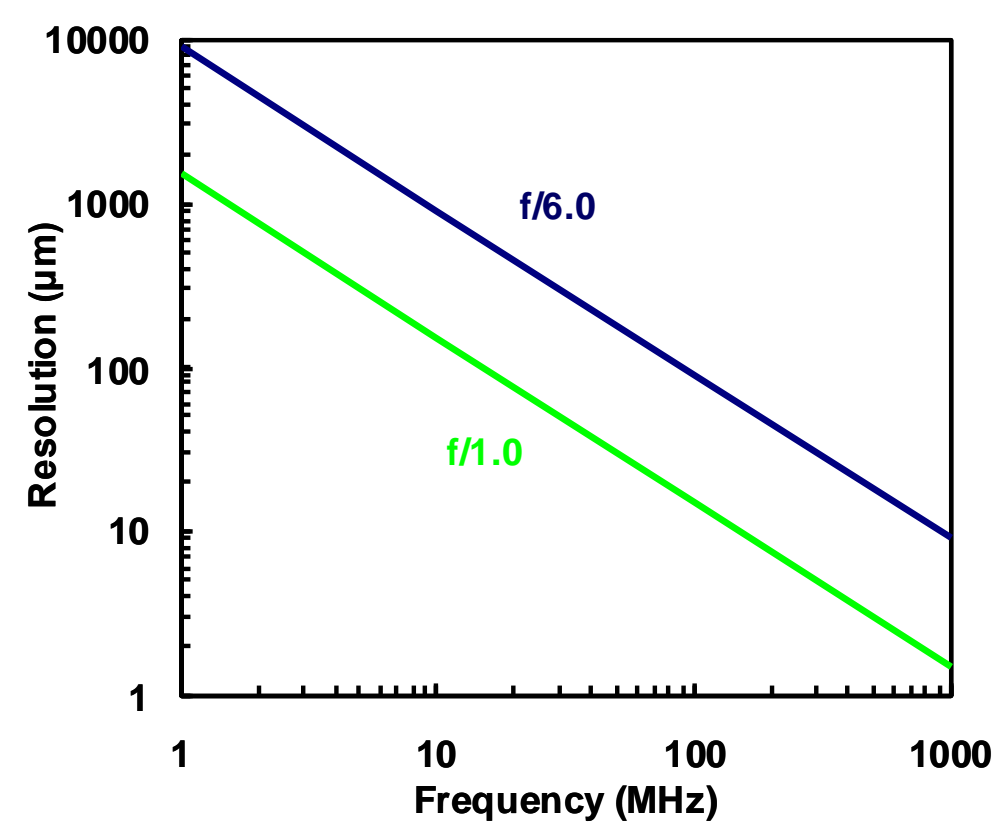

Figure 1.1: Change in ultrasound resolution with frequency for transducers with different f-numbers. 


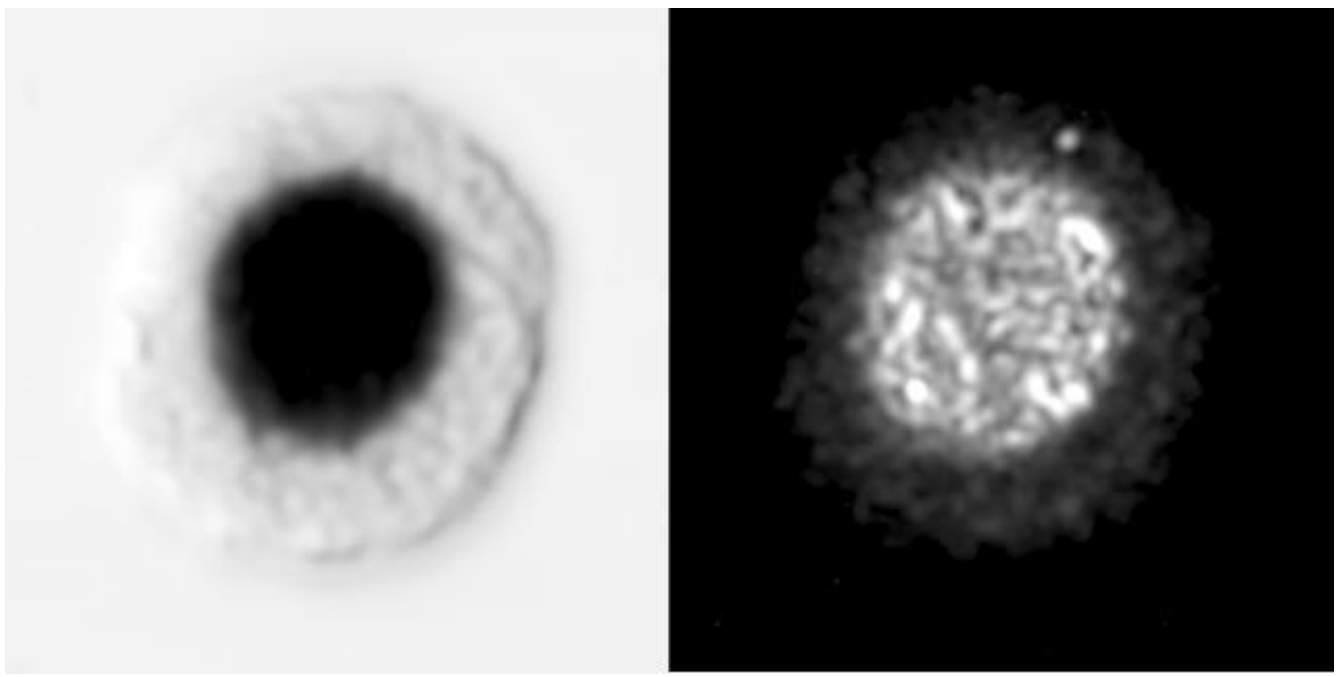

Figure 1.2: Ultrasound attenuation (left) and backscatter (right) images of a 1.4 diameter MCF7 spheroid measured using an $80 \mathrm{MHz}$ transducer. In the attenuation image, the dark areas indicate areas of strong attenuation, while in the backscatter image, the light areas indicate areas of strongest backscatter.

Ultrasound has enabled safe and relatively inexpensive visualization of structure through opaque materials and tissue with acceptable resolution and contrast. While ultrasound is primarily used for imaging, quantitative ultrasound (QUS) methods are becoming increasingly popular due to the ability to extract information about the sample noninvasively and non-destructively. QUS uses various signal processing methods to calculate and quantify the ultrasound spectrum that provide information about the frequency dependent backscatter signal, which provides information about the scattering sources within tissues. Spectral parameters derived include the spectral slope, mid-band fit and y-intercept [16-18]. These methods have been used to classify plaque $[19,20]$, classify scatter size and sound speed in tissue [21-23], detect changes in tissue due to apoptosis [24-27], differentiate 
cancers in mice [28], detect cancer in lymph nodes [29-31], and quantify blood cell aggregation [32-34]. Other QUS methods involve measurement of one or more acoustic properties such as the sound speed or attenuation, which can be used to differentiate tissue [35].

In the same way that clinical/high frequency ultrasound can be used to probe tissue, UHF acoustic microscopy can be used to probe single cells. The first acoustic microscope with sub-micron resolution was developed in 1973 by Lemons and Quate [36]; numerous studies on fixed and live cells have followed (e.g. [37-40]). At a frequency of $375 \mathrm{MHz}$, the lateral and axial resolutions are approximately $4 \mu \mathrm{m}$. Figure 1.3 shows a single MCF7 cell imaged with 375 and $1000 \mathrm{MHz}$ frequencies; the majority of the ultrasound backscatter occurs from the central region of the cell. The nuclear membrane and nucleolus are visible in the ultrasound attenuation image. At $1000 \mathrm{MHz}$, the resolution approaches $1 \mu \mathrm{m}$. Fine detail of the cell, including the nucleolus, nuclear membrane and organelles around the nuclear membrane are visible in the attenuation image (Figure 1.3). The backscatter image shows interference fringes that occur due to constructive/destructive interference between the signals from the substrate and cell surface. 

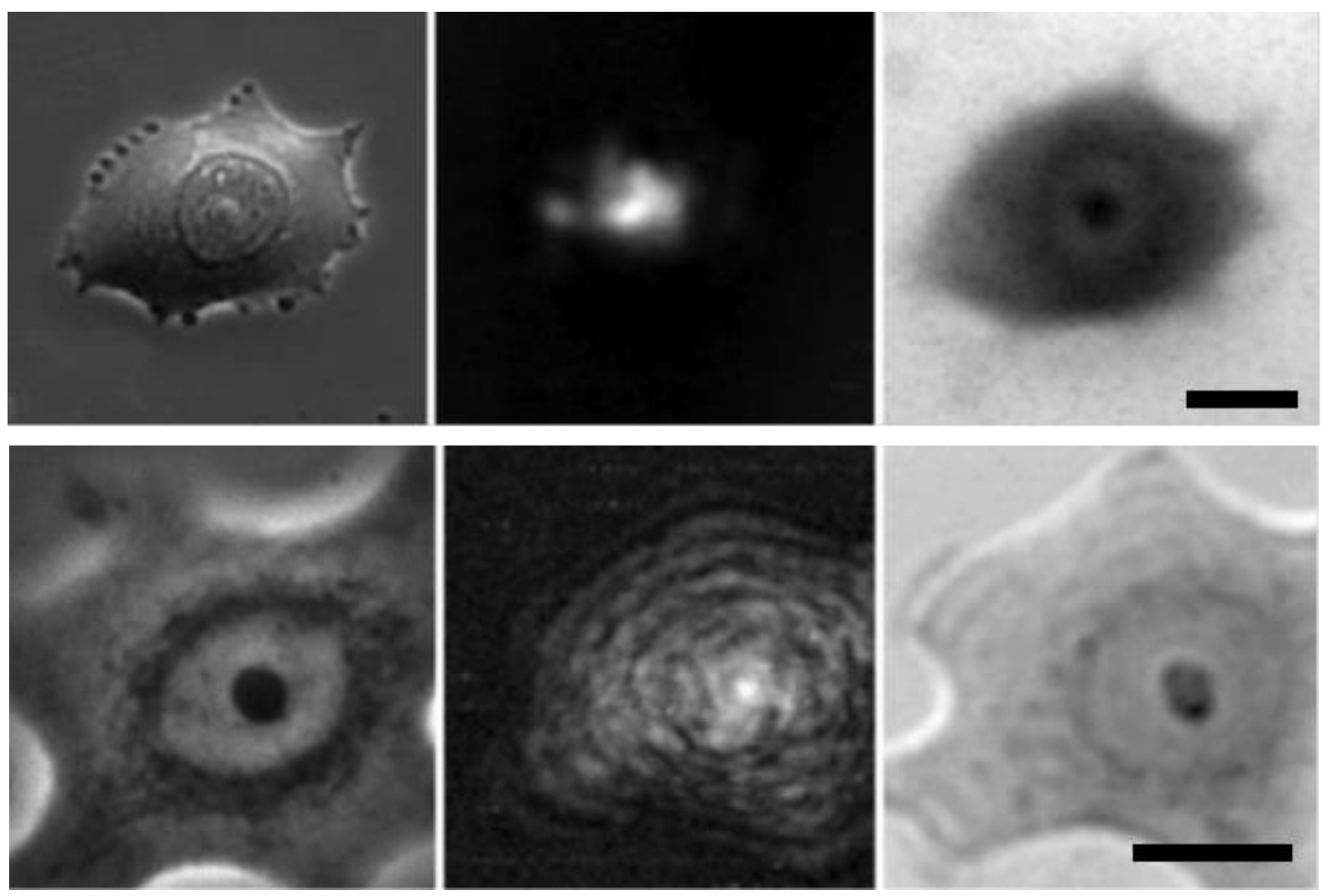

Figure 1.3: Optical (left), ultrasound backscatter (middle) and ultrasound attenuation (right) images of a single MCF7 cell using $375 \mathrm{MHz}$ (top) and $1000 \mathrm{MHz}$ (bottom). The scale bar is $15 \mu \mathrm{m}$.

Quantitative information about single cells can also be obtained through acoustic microscopy methods. The thickness, acoustic impedance and attenuation of single living cells can be inferred from interference fringes resulting from reflections from the cell surface and substrate [41-43]. Variations in the signal measured as a function of depth (called a $v(z)$ curve) or frequency (called a $v(f)$ curve) can be used to determine several properties of cells such as the thickness, sound speed, density, elasticity and attenuation [44-46]. Time resolved methods use echoes from the cell surface and substrate separated in time to determine the 
thickness, sound speed, acoustic impedance and attenuation of a cell [47-50]. All these methods require adherent cells, and in some cases, estimation of some cell properties to deduce other cell properties. Additionally, the measurements can be time consuming to measure a large sample size.

\subsection{Principles of Photoacoustics}

The photoacoustic effect was first discovered by Alexander Graham Bell in 1880 [51]. When he irradiated a selenium cell with modulated sunlight, he was able to hear sound waves. The requirements for the photoacoustic effect is that the object or material absorb optical energy, and the energy source must be modulated. The material rapidly absorbs the energy, resulting in an increase in temperature. This increase in temperature causes a rapid increase in pressure within the material, resulting in a thermoelastic expansion and the emission of a pressure wave. This wave is called a photoacoustic wave due to the optical stimulation method in which it was generated. While ultrasound and photoacoustics both involve ultrasonic pressure waves, the method of generation is different. In ultrasound, pressure waves travelling towards the sample are scattered, and this scattered signal is recorded. The scattering depends on the mechanical properties of the sample, or more specifically, the acoustic impedance mismatch between the sample and its surroundings. In contrast, a photoacoustic wave is generated due to optical absorption of energy; the photoacoustic wave amplitude depends on the optical absorption coefficient of the sample and the optical intensity, while features of the emitted wave depend on the mechanical 
properties of the sample. Figure 1.4 illustrates how the ultrasound and photoacoustic waves are generated from a single spherical homogeneous particle. An example pressure wave for a $20 \mu \mathrm{m}$ spherical homogeneous liquid is shown in Figure 1.5; it has a characteristic "N" shape as a function of time [52].

\section{A: Photoacoustics}

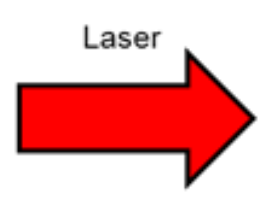

Particle

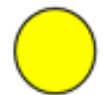

Before: Laser hits particle

\section{B: Ultrasound}

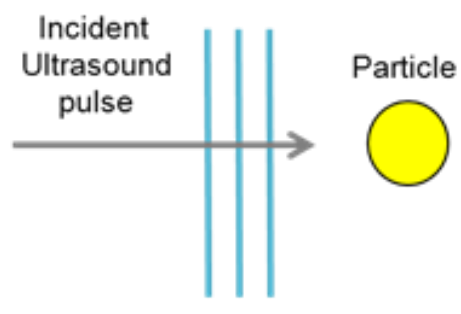

Before: Ultrasound hits particle
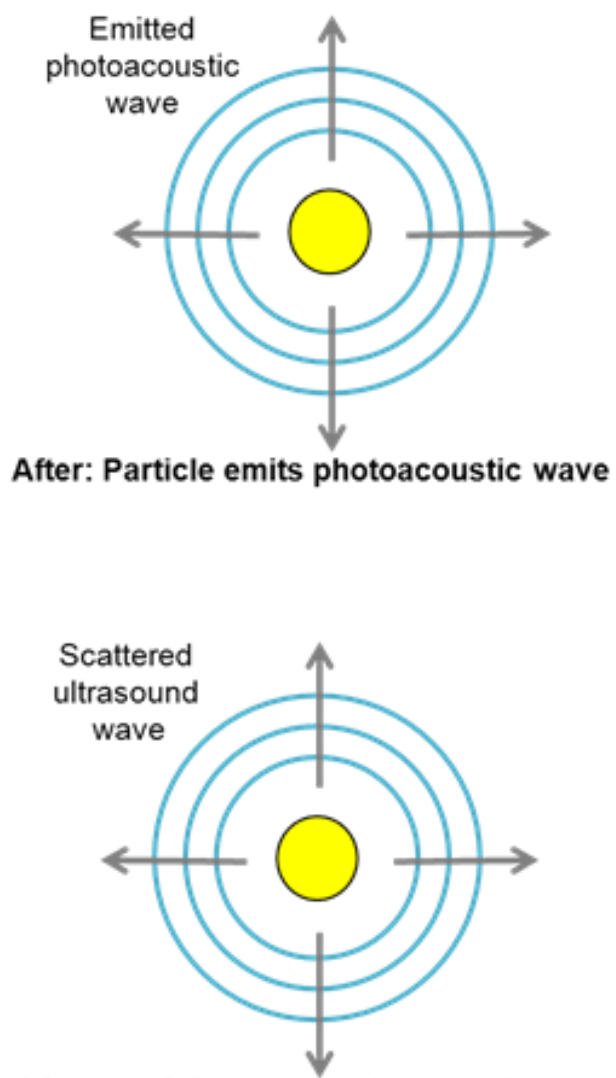

After: particle scatters ultrasound wave

Figure 1.4: Illustration of how photoacoustic and ultrasound waves are generated from a single spherical particle. In photoacoustics, the particle is irradiated by a laser, absorbs the energy and emits a pressure wave with ultrasonic frequencies. In ultrasound, the particle is insonified by incident pressure waves, which then reflect from the particle. 


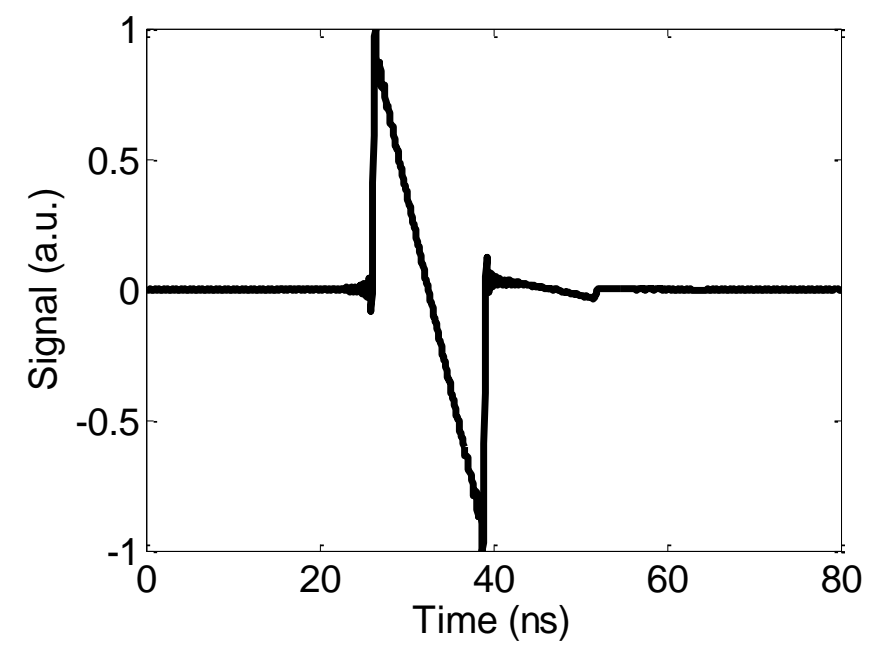

Figure 1.5: The theoretical photoacoustic time domain signal of a $20 \mu \mathrm{m}$ diameter liquid sphere with a sound speed of $1560 \mathrm{~m} / \mathrm{s}$ and a density of $1050 \mathrm{~kg} / \mathrm{m}^{3}$.

Unlike ultrasound, the emitted photoacoustic wave from an object is inherently broadband [53], where the frequencies of the wave depend on the size, shape and sound speed of the object. The general equation describing the emission of a photoacoustic pressure wave upon irradiation of optical energy $H$ is

$$
\frac{1}{c^{2}} \frac{\partial^{2} p}{\partial t^{2}}-\nabla^{2} p=\frac{\beta}{C_{p}} \frac{\partial H}{\partial t}
$$

where $p$ is the pressure, $t$ is time, $c$ is the sound speed, $\beta$ is the thermal expansion coefficient, and $C_{p}$ is the heat capacity in the absorbing medium [53]. The left hand side of equation 1.1 is the wave equation, while the right hand side is an additional time-dependent term to account for the absorption of optical energy. Analytical solutions of this equation exist for simple homogeneous geometries such as spheres, cylinders and infinite planes [52, 54-58], 
however numerical methods must be used for heterogeneous systems or other geometries. Further details on these numerical methods are described in chapters two and three.

For efficient generation of photoacoustic waves, two conditions should be satisfied: 1) Thermal confinement, where the optical energy is entirely absorbed before heat conduction occurs, and 2) Stress confinement, where the optical energy is absorbed before propagation of the pressure wave occurs. These conditions can be fulfilled if the optical pulse width $t$ is shorter than the thermal and stress confinement times,

$$
t_{\text {stress }}=\frac{d}{c}, \quad t_{\text {thermal }}=\frac{d^{2}}{4 \alpha},
$$

where $d$ is the characteristic length scale of the object, $c$ is the sound speed and $\alpha$ is the thermal diffusivity [59]. When these conditions are not met, heat conduction and propagation of the pressure wave occur before the energy is completely absorbed; this results in poor photoacoustic signal generation and low amplitude pressure waves. As an example, a $20 \mu \mathrm{m}$ diameter sphere composed of water requires $\mathrm{t}_{\text {stress }}<13 \mathrm{~ns}$ and $\mathrm{t}_{\text {thermal }}<0.7 \mathrm{~ms}$, while a $1 \mu \mathrm{m}$ diameter liquid sphere made of perfluorohexane requires $\mathrm{t}_{\text {stress }}<2 \mathrm{~ns}$ and $\mathrm{t}_{\text {thermal }}<8 \mu$ s. Solids generally have lower confinement times; a $1 \mu \mathrm{m}$ diameter solid sphere made of polystyrene requires $\mathrm{t}_{\text {stress }}<0.4 \mathrm{~ns}$ and $\mathrm{t}_{\text {thermal }}<4 \mu \mathrm{s}$. In most cases, the thermal confinement time is much larger than the stress confinement time.

Contrast in ultrasound imaging depends on acoustic impedance mismatches within the medium; the contrast associated with photoacoustic imaging results from the optical absorption properties of the medium. Only components that absorb the optical energy will 
emit a photoacoustic wave. In photoacoustic imaging, the sample is irradiated by a laser, and an ultrasound transducer is positioned to detect the resulting photoacoustic pressure waves. Within the human body, only a few structures strongly absorb optical energy throughout the visible and near-infrared spectrum. One is blood (from the hemoglobin), and the other is melanin (a pigment found in skin and hair cells). photoacoustic imaging methods generate high contrast images of the vasculature due to the high optical absorption properties of blood relative to the negligible absorption properties of surrounding tissue. Photoacoustic imaging has produced high resolution imaging of vasculature in-vivo [60-62], and can detect changes in blood flow in functional photoacoustic imaging [63, 64]. Frequencies between 1-60 MHz are typically used for photoacoustic imaging. The penetration depth is limited to approximately 1-2 cm due to scattering of the incoming photons. Exogenous photoacoustic contrast agents such as nanoparticles or dyes can be introduced in-vivo to enhance photoacoustic signals from specific targets such as tumors $[65,66]$.

The resolution of ultrasound pulse-echo imaging systems is limited by the frequency of the transducer; high resolution imaging requires high frequencies. With photoacoustic imaging, standard clinical and high frequency transducers (up to about $60 \mathrm{MHz}$ ) have been used along with a focused laser to achieve high resolution imaging. This is the basis for photoacoustic microscopy (PAM). In these systems, the laser is typically focused to a micron-sized spot [64], although resolution near the diffraction limit of $200 \mathrm{~nm}$ has been achieved [67]. Many different PAM designs exist. The laser can be positioned opposite the transducer [68-70], integrated into the transducer [71-73], positioned directly beside the 
transducer [74-76], with many other configurations possible [60, 77-79]. In most cases, the lateral resolution is limited by the laser spot size, which itself is related to the numerical aperture (NA) of the optical objective. These microscope systems can provide high resolution images of single cells, capillaries and tissue structures with sub-micron resolution [64]. Alternatively, high resolution photoacoustic imaging can also be performed using a weakly focused laser and high frequency transducers; in this case, the resolution is limited by the focal spot of the receiving transducer [80]. Example photoacoustic images of a single melanoma cell (containing melanin pigment) imaged with a $532 \mathrm{~nm}$ laser focused to a $5 \mu \mathrm{m}$ spot and imaged with successively higher frequencies is shown in Figure 1.6. The laser spot size remained the same for all images, and increased detail is observed as the transducer frequency is increased from $200 \mathrm{MHz}$ (Figure 1.6B, $8 \mu \mathrm{m}$ transducer resolution) to 1200 $\mathrm{MHz}$ (Figure 1.6D, $1 \mu \mathrm{m}$ transducer resolution). The main advantages to using high frequency transducers are 1) axial resolution that approaches $1 \mu \mathrm{m}$ at $1000 \mathrm{MHz}$, and 2) the wide bandwidth that can be used for a quantitative analysis of the photoacoustic signals that cannot be achieved with lower frequency photoacoustic microscopes. 


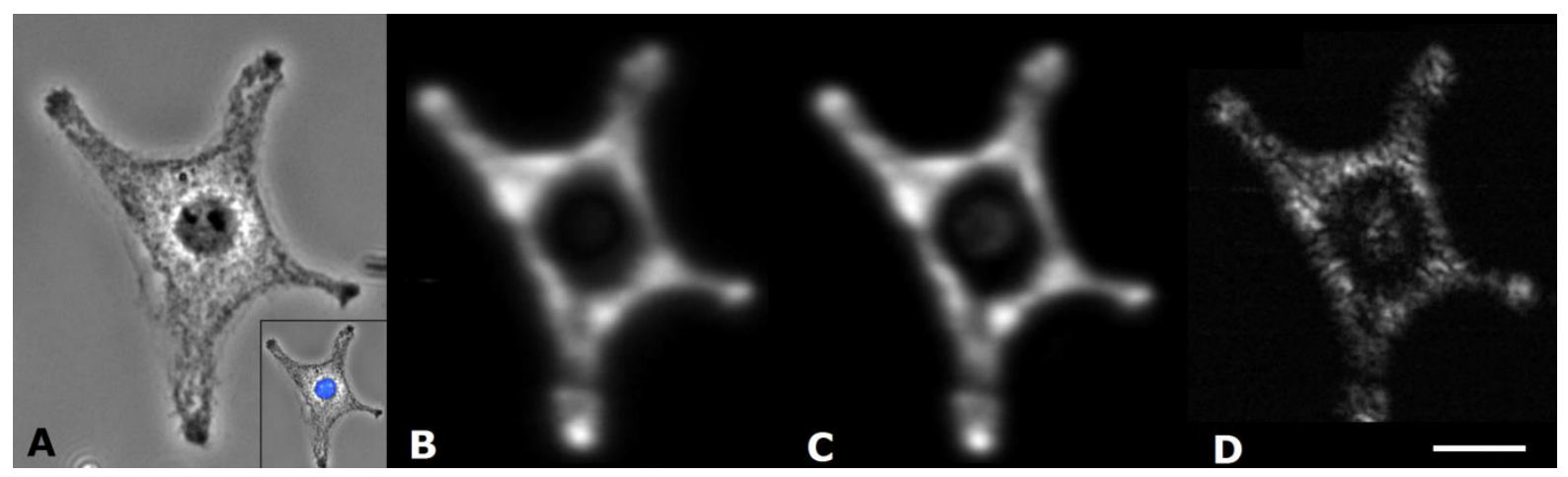

Figure 1.6: Photoacoustic images of a fixed B16-F1 melanoma cell imaged with progressively higher transducer frequencies. The laser spot size remained the same at approximately $5 \mu \mathrm{m}$; therefore the imaging resolution is a function of the transducer, not the laser spot. (A) Optical image with fluorescence inset, (B) Photoacoustic image using $200 \mathrm{MHz}$, (C) $375 \mathrm{MHz}$ and (D) $1200 \mathrm{MHz}$. The scale bar is $30 \mu \mathrm{m}$. (Figure adapted from [80])

Quantitative photoacoustic methods (QPA) are a recent development that can give information about the sample examined. The method is similar to QUS discussed in section 1.2, where features of the power spectrum are examined and correlated to specific sample properties. Micron-sized samples such as cells or thin tissue slices typically examined in a PAM have photoacoustic waves with significant spectral features over $100 \mathrm{MHz}$ [55, 81], which are outside of the bandwidth of the transducers normally used in most photoacoustic systems developed. In order to perform a quantitative analysis of the photoacoustic signals from small samples such as cells, transducers with center frequencies over $100 \mathrm{MHz}$ and bandwidths in the hundreds of $\mathrm{MHz}$ must be used. In these cases, the signals measured from ideal spheres no longer have the classic "N" shape; the signal is distorted due to the limited bandwidth of the transducer. Figure 1.7 shows the time domain signal of a $20 \mu \mathrm{m}$ diameter 
liquid sphere from Figure 1.5 when measured using a limited bandwidth of 100-500 MHz, simulated using a bandpass filter. Limited bandwidth ultimately reduces the signal amplitude as the lower frequency content containing most of the wave energy is not detected.

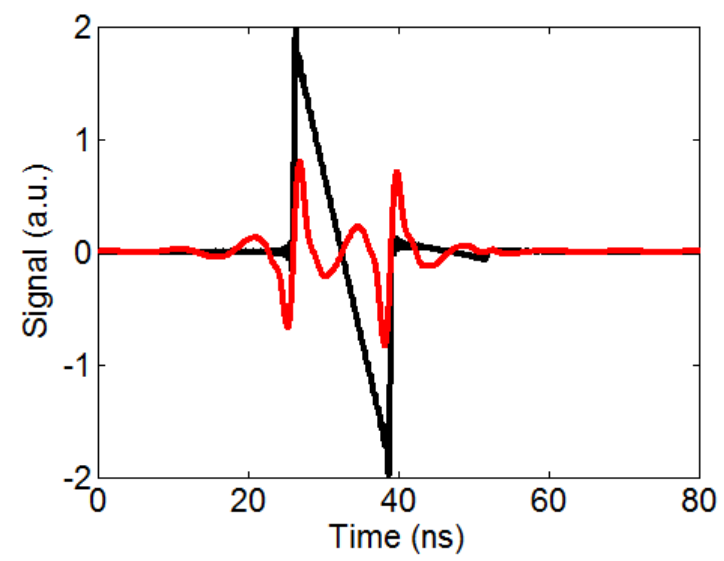

Figure 1.7: The theoretical photoacoustic signal of the $20 \mu \mathrm{m}$ spherical particle shown in Figure 1.5 (black) when filtered with a 100-500 MHz bandpass filter (red), simulating the limited bandwidth of a typical UHF transducer.

\subsection{Probing single cells with ultrasound and photoacoustics}

Cells are the building blocks of life. They are typically 5-30 $\mu \mathrm{m}$ in diameter, enclosing a cytoskeleton and various organelles such as a nucleus, mitochondria, lysosomes and vacuoles. Complex processes occur within the cell on a micro and molecular level, particularly in response to stimuli or changes in the chemical environment. The composition of each type of cell can be different, and various biological processes can alter the chemical and structural balance of the cells [82-85]. Ultrasound has been used to non-invasively classify tissue types according to differences in the measured sound speed or attenuation 
[35]. Changes in the cell properties enable identification of these biological processes in bulk tissue such as apoptosis using clinical and high frequency ultrasound [25, 86-89].

Many tools exist for probing cell properties at the single cell level, which include microrheology [90, 93], micropipette aspiration [94, 95], optical and magnetic tweezers [96, 97] atomic force microscopy [98-105] and ultrasound/acoustic microscopy [11, 47, 50, 106]. Changes in the cell properties can be used to differentiate cell types, and also detect malignancy. For example, changes in the elasticity has been observed between benign and malignant breast cancer cell lines [93, 103, 107]. Acoustic microscopy has been used to probe the properties of single adherent cells, however the cell properties can be dependent on the substrate the cell is grown on, and the pressure exerted by neighbouring cells. A method to examine single cells in suspension non-invasively, whether cells that are naturally in suspension such as blood cells, or adherent cells that have been detached from the substrate, does not exist. Examining cells in suspension provides advantages to those grown on substrates, as external stresses that can alter the cellular properties are removed from the system.

Clinical to high-frequency $(<100 \mathrm{MHz})$ photoacoustic and ultrasound imaging and quantitative methods cannot resolve individual cells. When the ultrasound wavelength is much larger than the cell, as it is in the 1-20 MHz regime, Rayleigh scattering occurs. The measured ultrasound signal is a superposition of signals resulting from scattering from many sub-resolution objects within the interrogating region. As described in the previous sections, achieving imaging resolution in the micron-range for single cells requires near-GHz range 
frequencies for ultrasound and photoacoustics. For QPA and QUS analysis of single cells, the wavelength should be similar to the size of the cell. The ultrasound wavelength is approximately $15 \mu \mathrm{m}$ at $100 \mathrm{MHz}$ and $3 \mu \mathrm{m}$ at $500 \mathrm{MHz}$, whereas cells can range from 5-30 $\mu \mathrm{m}$, depending on the cell type. When probing spherical cells, the resulting ultrasound and photoacoustic power spectrum have periodically varying minima and maxima where the location and spacing between minima and maxima depend strongly on the size, shape and sound speed of the cell. Aside from the nucleus, most organelles are sub-micron and distributed throughout the cytoplasm. Studies have shown that the nucleus has a slightly different sound speed [108] and attenuation [106] than the surrounding cytoplasm, and it may affect how the ultrasound scatters from the cell.

Photoacoustics requires that the cell absorbs optical energy. Aside from cells with endogenous optical absorbers such RBCs (hemoglogin) and melanocytes (melanin), exogenous optical absorbers such as nanoparticles or dyes must be added to cells to facilitate photoacoustic wave generation. The DNA/RNA in cells can also be used as an endogenous absorbing agent, provided they are irradiated at their peak absorption of $266 \mathrm{~nm}$ [73]. Melanoctyes contain an abundance of melanin particles distributed throughout the cytoplasm, but not within the nucleus. Nanoparticles also tend to accumulate throughout the cytoplasm, avoiding the nuclear area [109]. Generally nuclear penetration is difficult even for the smallest nanoparticles; dyes provide better flexibility with selectively staining the cell. Membrane-permeable dyes such as Hoechst (which targets the nucleus) and neutral red (targets many components, particularly lysosomes) can be used for live-cell analysis. Non- 
membrane permeable dyes such as Trypan blue can only be used with dead cells that have lost membrane integrity. Alternatively, cells can be temporarily permeabilized with the addition of a detergent such as Triton $\mathrm{X}-100[110,112]$. This allows molecules to pass through the cell membrane that would normally be too large. By using specific optical absorbing agents, specific regions of the cell can be targeted. Also, optical spectroscopy methods can be used to target different chromophores. By varying the irradiating wavelength, the different types of chromophores will generate photoacoustic waves, thus selectively targeting specific components of a sample [113-117].

\subsection{Signal normalization}

The ultrasound and photoacoustic signals as recorded include the signal from the sample plus effects due to attenuation in the medium, the transducer transfer function, and other artifacts [118]. For the most part, these effects can be removed by measuring a reference spectrum. For ultrasound pulse echo measurements, this is traditionally performed by measuring the reflection from a highly reflecting material such as glass or quartz at the same distance as the sample. Measurement of a reference for photoacoustics is not so straightforward. In photoacoustics, the pulse undergoes one-way travel from the sample to the transducer, while ultrasound pulse echo measurements undergo two-way travel; the attenuation is less for a photoacoustic wave, which can be substantial at frequencies over 100 MHz. Also, the photoacoustic wave is influenced by the transducer transfer function once (incoming only), as opposed to twice for pulse-echo ultrasound measurements. Finally, the 
photoacoustic emission from any source is inherently broadband, emitting a broad frequency range that depends on the size, sound speed and optical absorption characteristics of the sample. An ultrasound transducer is only recording a limited bandwidth of all frequencies emitted. In contrast, in ultrasound a sample is irradiated by a limited bandwidth of frequencies, where the reflected wave depends on the acoustic properties of the sample. Given these factors, the pulse echo reference method should not be used for photoacoustic normalization.

A method to acquire the photoacoustic reference signal is to measure the photoacoustic emission from a sample that has a nearly flat spectrum over a wide frequency range. This would require a narrow pulse emission in time, ideally a delta function. The material should be flat, highly absorbing and very thin. A $200 \mathrm{~nm}$ thick gold film deposited onto glass meets these criteria was used as a photoacoustic reference. The theoretical spectrum using an infinite plane model [119] from this gold film is shown in Figure 1.8. The amplitude change in the spectrum is approximately $1.5 \mathrm{~dB}$ from 1 to $500 \mathrm{MHz}$, and $3 \mathrm{~dB}$ from 1 to $1000 \mathrm{MHz}$. While there is a slight decrease in the signal with frequency, the spectrum is sufficiently flat with no significant spectral features.

The photoacoustic signal measured using this gold film source, along with a comparison to an ultrasound pulse echo measured from glass using a $375 \mathrm{MHz}$ center frequency transducer is shown in Figure 1.8. The ultrasound - 6 dB bandwidth is $44 \%$ (285$450 \mathrm{MHz}, \Delta \mathrm{f}=165 \mathrm{MHz}$ ), while the photoacoustic $-6 \mathrm{~dB}$ bandwidth is $57 \%$ (245-460 MHz, $\Delta \mathrm{f}=215 \mathrm{MHz}$ ); the photoacoustic bandwidth is wider by $50 \mathrm{MHz}$. The ultrasound pulse 
propagates twice the distance as the photoacoustic wave, and therefore undergoes twice the attenuation. In practice, the usable bandwidth is slightly wider than the $-6 \mathrm{~dB}$ bandwidth; typically frequency ranges between $200-500 \mathrm{MHz}$ can be detected with the $375 \mathrm{MHz}$ transducer.
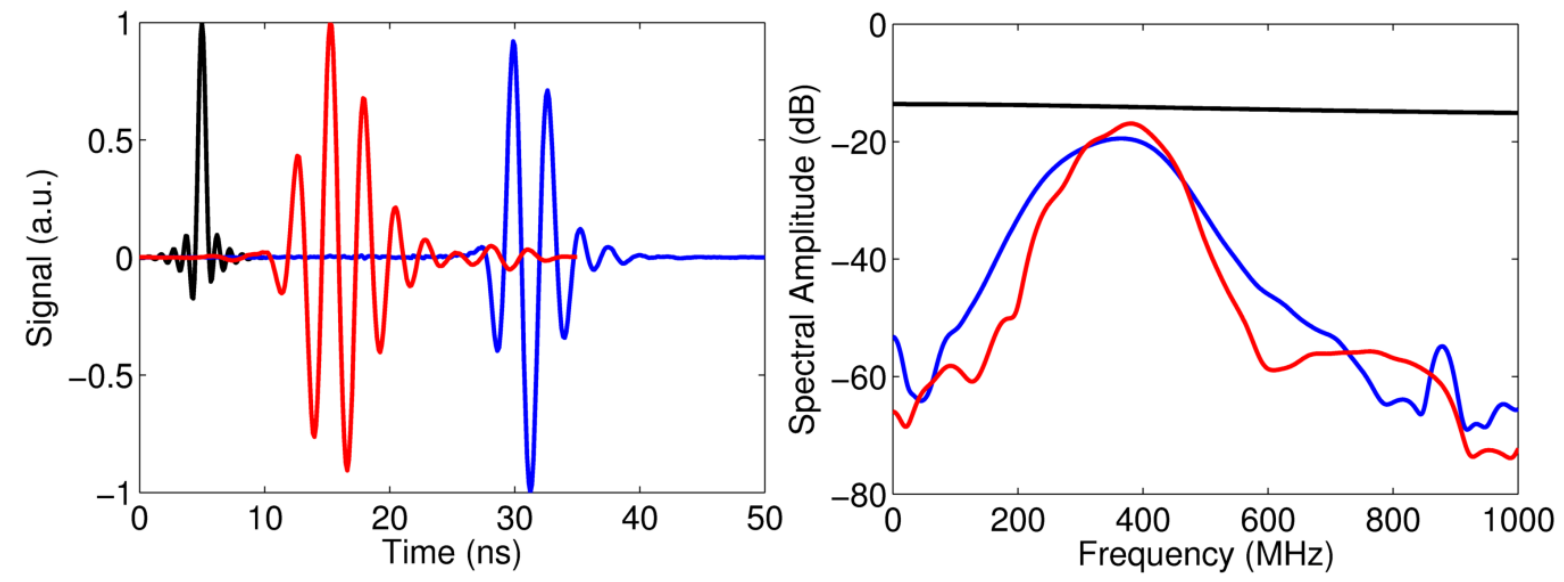

Figure 1.8: The time domain signal (left) and spectrum (right) of a $375 \mathrm{MHz}$ transducer used for photoacoustics (blue) and ultrasound (red), along with the theoretical spectrum for a $200 \mathrm{~nm}$ thick gold film (black). The $-6 \mathrm{~dB}$ bandwidth for ultrasound was $165 \mathrm{MHz}$ (285-450 MHz), but the same transducer used for photoacoustics had a $215 \mathrm{MHz}$ bandwidth (245-460 MHz).

\subsection{Modeling ultrasound and photoacoustic interactions}

Analytical solutions of the interaction and scattering of ultrasound with particles is well established, with publications dating back to the 1950's [120-122]. These theories have been recently validated using frequencies up to about $60 \mathrm{MHz}$ through a comparison of measurements of micron-sized polystyrene beads and single cells [108, 118, 123-126]. Good agreement between theory and measured were observed. Analytical solutions of optically20 
induced photoacoustic waves was developed in the late 1980's [55]. These solutions were verified on mm-length scales using frequencies up to about $20 \mathrm{MHz}$, but no measurements have been performed on micron-sized particles, which requires frequencies over $100 \mathrm{MHz}$. To date, only two photoacoustic microscopes with capability of using frequencies over 100 MHz exist in the world [127-129].

Spherically symmetric homogeneous particles are assumed for the ultrasound and photoacoustic analytical solutions. These solutions can break down for asymmetric particles, heterogeneous particles, or for particles near boundaries. In these cases, numerical methods must be used. Finite element methods (FEMs) split the problem into small components which are then iteratively solved according to initial conditions. As with the ultrasound analytical models, several studies have used FEMs to solve ultrasound scattering from micron-sized particles such as cells and contrast agents [126, 130-133]. Numerical studies have examined the photoacoustic signals generated from tissue [134-137], however these are typically complex systems involving photon diffusion, heat transfer and stress equations and require extensive computational resources to complete. Few studies, if any have modeled photoacoustic wave generation from micron-sized particles.

\subsection{Goals and specific aims}

Ultrasound scattering theory is well developed; QUS analyses of cells and particles have been studied using UHF ultrasound since the mid-1970's. In contrast, there have been limited quantitative analyses of photoacoustic systems. Photoacoustic imaging has been used 
to image tissue and cells, but quantitative photoacoustic methods have never been used for single cells. The goal of this dissertation is to develop a better understanding of UHF photoacoustic waves, their interactions with cells and how this can be used at a clinical level to help detect and diagnose disease such as CTCs.

\section{Hypothesis:}

The signal amplitude and spectral features of UHF photoacoustic waves can be used to characterize and differentiate cell types, and pathological states of cells.

\section{Specific aims:}

1) Develop numerical methods of UHF photoacoustic wave generation from particles in the micron-size range.

2) Validate numerical methods using experimentally measured photoacoustic signals.

3) Demonstrate that photoacoustic methods can identify morphological and pathological changes in RBCs.

4) Differentiate cell types in blood using photoacoustic signals.

\subsection{Overview of dissertation}

The research presented in this dissertation is the first reported use of photoacoustic frequencies over $100 \mathrm{MHz}$ on single cells. An analysis of measured signals from single cells and particles, as well as a thorough comparison to theoretical models are discussed in chapters 2-4. In chapter 5, photoacoustic measurements are used for specific cell 
identification.

Chapter 2 develops the analytical and numerical methods of photoacoustic wave emission from micron-sized perfluorocarbon (PFC) emulsions containing gold nanoparticles (AuNPs). The NPs facilitate photoacoustic emission, as the PFC liquid has negligible absorption in the visible and infrared wavelengths. The thermoelastic expansion model used to describe the photoacoustic signals generated from single emulsions is compared to measured signals. The analytical solution to the thermoelastic expansion model gave valid solutions for symmetric and homogeneous particles such as the emulsions, however it breaks down in other situations such as particles with inhomogeneities or near boundaries. A finite element model (FEM) was developed to simulate the photoacoustic signals emitted from particles in these cases and obtain the frequency content. The numerical simulations were compared to measurements for model validation.

In Chapter 3, the photoacoustic spectral features of PFC emulsions were compared to theoretical models to extract the particle size. The emulsions are an ideal particle to verify the quantitative photoacoustic methods, as they are spherically symmetric and homogeneous with known physical properties. The sizes determined through photoacoustic methods were compared to ultrasound and optical methods to validate the methodology.

Chapter 4 investigated photoacoustic signals from RBCs. RBCs have a unique biconcave shape that is traditionally difficult to model due to its asymmetry; most simulations approximate the RBC shape as a sphere or oblate ellipsoid. The FEM developed in chapter 2 was used to examine how photoacoustic signals vary as a function of RBC size, orientation 
and morphology.

Chapter 5 used the photoacoustic signal amplitude and features in the power spectrum to classify cell types. The location of the spectral features are dependent on the ratio of the size to sound speed, while the photoacoustic amplitude is related to the absorption coefficient of the cell. Cells that have inherent optical absorption (such as RBCs and melanoma cells) can be easily differentiated by these measurements; other cells such as AML must be dyed prior to investigation.

Chapter 6 summarizes the results, clinical significance and future work. Appendix A summarizes the literary contributions resulting from research performed during my $\mathrm{PhD}$ studies that both directly and indirectly contributed to this dissertation. 


\section{Chapter 2}

\section{A Photoacoustic finite element model to}

\section{describe spectral features of micron-sized}

\section{particles}

\subsection{Abstract}

The photoacoustic signal generated from micron-sized particles is determined by physical attributes of the particle such as size and sound speed. The frequency content of these signals can be used to obtain the physical attributes, however the wave generation and propagation can be altered by nearby structures. To determine the frequency content of the photoacoustic signals generated, and assess the change in the photoacoustic signals from particles near structures, a finite element model (FEM) was developed to model the photoacoustic wave propagation from particles in various configurations: a particle in suspension surrounded by water, a particle on a reflecting boundary (which could be a vessel or other rigid structure), and a particle with an absorbing shell and non-absorbing core. Physical aspects normally important to photoacoustic FEMs such as photon transport, heat 
transfer and stress/strain equations were neglected to reduce complexity and improve computational time, as the goal was to obtain the frequency content of the wave. Perfluorocarbon (PFC) droplets 3-5 $\mu \mathrm{m}$ in diameter containing gold nanoparticles (to facilitate optical absorption) were used as they represent particles; they are spherical, homogeneous, optically translucent and their physical properties are known. They are also being investigated as potential photoacoustic/ultrasound contrast agents and therapeutic delivery vehicles (theranostic agents) in-vivo due to their versatility and biological compatibility. High-frequency photoacoustic measurements (over $100 \mathrm{MHz}$ ) of single droplets in suspension and on a glass substrate were compared directly to the FEM and analytical models to investigate how the photoacoustic power spectrum features change with boundary conditions. Periodically varying minima and maxima occur throughout the spectrum; for a $3.1 \mu \mathrm{m}$ droplet in suspension, the frequency location of the spectral minima between the FEM and analytical solution were in good agreement to measured values (3.3 MHz standard deviation). For a droplet on a reflecting boundary, extra spectral minima were observed which were correctly reproduced using the FEM. The average standard deviation of the location of the spectral features between measured and the FEM for a droplet on a glass substrate was $1.4 \mathrm{MHz}(2.8 \mu \mathrm{m}$ diameter $), 6.1 \mathrm{MHz}(3.1 \mu \mathrm{m}), 7.6 \mathrm{MHz}(3.6 \mu \mathrm{m})$ and 8.5 $\mathrm{MHz}(3.6 \mu \mathrm{m})$. These results demonstrate that the power spectrum changes depending on if a droplet is near a boundary, and the analytical model cannot correctly predict the spectral features. The FEM could be used in cases where a particle is irregular, near boundaries or has heterogeneous optically absorbing areas. 


\subsection{Introduction}

The photoacoustic effect is the emission of a pressure wave from a structure after irradiation by an energy source, typically a laser. For spatial scales of interest in biomedical photoacoustic imaging, the frequency of the wave is generally in the ultrasonic range. This wave is called a photoacoustic or optoacoustic wave due to the laser-induced method in which it was generated. Photoacoustic imaging requires an optical absorbing structure in the medium for a wave to be generated. Most tissue has negligible absorption throughout the visible and infrared spectrum where photoacoustic imaging is commonly used. The main absorbing structure in tissue is the blood and the main chromophore in the blood is haemoglobin. This endogenous source of contrast has been exploited in-vivo for the detection of vascular tumours [138] photoacoustic tomography [65] with functional imaging [139], and imaging the vasculature $[114,140]$. Photoacoustic contrast agents such as molecular agents or nanoparticles can be introduced into the bloodstream as contrast agents to help identify breast and prostate tumors [141-143]. Recently, nanoparticles (NPs) have been incorporated into perfluorocarbon emulsions where they can be used as theranostic agents $[144,145]$. At low laser energies, the emulsions act as photoacoustic contrast agents. At higher energies, the PFC liquid vaporizes to a gas state. The conversion to a gas state the newly created microbubbles can act as ultrasound contrast agents [146]. Additionally, a chemotherapeutic payload can also be incorporated into the droplets for localized drug delivery [147]. The optical absorbing nanoparticles are a necessary component for the generation of photoacoustic signals, as PFC liquids have negligible absorption in the visible 
and near-infrared wavelengths [148]. When irradiated with sufficient optical energy, the NPloaded micron-sized droplets emit photoacoustic signals. These contrast agents have a unique signature signal compared to other photoacoustic sources in tissue, and can be used to differentiate and quantify the emulsions present in a region. Since this signature is more readily differentiated in the frequency domain representation of the photoacoustic signal, a model that can accurately represent the frequency content of the signal is important for agent optimization.

When a micron sized particle absorbs energy, it undergoes a rapid thermoelastic expansion that results in emission of a photoacoustic wave in the ultrasonic frequency range. Neglecting viscosity and thermal conduction, the photoacoustic waves generated due to absorption of optical energy $H$ (such as a laser) is the solution to the inhomogeneous wave equation

$$
\frac{1}{c^{2}} \frac{\partial^{2} p}{\partial t^{2}}-\nabla^{2} p=\frac{\beta}{C_{p}} \frac{\partial H}{\partial t}
$$

where $p$ is the pressure, $t$ is time, $c$ is the sound speed, $\beta$ is the thermal expansion coefficient, and $C_{p}$ is the heat capacity [53]. Analytical solutions for the generation of photoacoustic waves have been derived for simple geometries just as spheres [55]. Assuming the pressure wave and energy source vary with time as $\exp (-i \omega t)$, the solution to equation $2-1$ for the photoacoustic waves generated from a liquid homogenous spherically symmetric particle is 


$$
P(f)=i\left(\frac{\mu_{a} I_{0} c_{d} \beta}{4 \pi C_{p}(r / a)}\right) \frac{(\sin q-q \cos q) / q^{2}}{\left(1-\frac{\rho_{d}}{\rho_{f}}\right)(\sin q / q)-\cos q+i \frac{c_{d}}{c_{f}} \frac{\rho_{d}}{\rho_{f}} \sin q}
$$

where $f$ is the frequency, $a$ is the particle radius, $\mu_{a}$ is the optical absorption coefficient, $I_{0}$ is the incident laser intensity, $\rho$ is the density, the subscripts $d$ and $f$ refer to the droplet and fluid, respectively, and $q=2 \pi f a / c_{\mathrm{d}}$ [54]. Good agreement has been found between this solution and measured photoacoustic signals for liquid homogeneous spheres [55]. The analytical solution breaks down for complex configurations such as multi-particle systems, particles near boundaries, or particles that have a heterogeneous composition. Tools such as the k-wave toolbox [149], Field II [150, 151], or COMSOL Multiphysics can be used to simulate the photoacoustic signal propagation. Some tools use finite element models (FEMs) where the system is decomposed into smaller components which are then iteratively solved. Recently, photoacoustic FEMs have examined temperature and displacement effects in tissue $[152,153]$, photoacoustic tomography reconstruction $[154,155]$ and photoacoustic propagation in tissue [156]. FEMs probing photoacoustic measurements are typically complex, requiring the integration of several different physical phenomena such as photon transport/scattering, thermal transport, stress/strain relations and acoustic propagation, which increases the computational resources required to solve a problem [157].

A simplified FEM was developed to investigate the frequency content of photoacoustic waves generated in cases where the analytical solution cannot be used. This model neglects photon diffusion/scattering, thermal transport and stress/strain relations that 
are commonly used in photoacoustic FEMs. The model initiates immediately after the particles are irradiated and absorb the laser energy. Assumptions are that the particle uniformly and instantly absorbs the laser energy, resulting in a pressure increase relative to the surrounding non-absorbing areas. The FEM then solves for the propagation of the acoustic waves due to the pressure differential within the system using the wave equation

$$
\frac{1}{c^{2}} \frac{\partial^{2} p}{\partial t^{2}}-\nabla^{2} p=0 .
$$

The FEM solution was solved for three different scenarios: 1) a droplet in suspension, 2) a droplet on a highly reflecting boundary and 3) a droplet in suspension but with nonhomogenous pressure distribution (emulating non-uniform optical absorption within the droplet). During optical irradiation, spherically symmetric pressure waves are emitted from the droplet. For a droplet in suspension (scenario 1), these waves simply radiate outwards and attenuate within the surrounding medium. This solution can be compared directly to the analytical solution (equation 2-2). For a droplet near a boundary (scenario 2), waves emitted from the droplet will be reflected by the boundary back into the droplet and towards the receiving transducer. Scenario 3 simulates a non-homogeneous droplet with a thin ring of optical absorbing material surrounding a non-absorbing liquid core. This simulates contrast agents where the optical absorbing material is contained in the shell instead of the core [158, 159]. Scenarios 1 and 2 were compared to experimental photoacoustic measurements of micron-sized PFC droplets containing NPs as optical absorbers.

This study shows that the FEM gives matching solutions to the analytical equation for 
ideal cases such as a droplet in suspension, and correctly predicts the extra spectral features observed from particles on a boundary where the analytical model cannot be used. The FEM can be applied for more complex systems where the analytical solution cannot be used, providing insight into how the frequency content of the photoacoustic waves are modified as a function of the droplet/boundary characteristics.

\subsection{Methods}

\subsubsection{Perfluorocarbon Droplets}

$\mathrm{PbS}$ nanoparticles were synthesized [160], coated with silica [161, 162], then fluorinated for miscibilization into PFP (Synquest, USA) [163]. PbS-loaded PFP droplets were prepared using $5 \mathrm{~mL}$ deionized water, $0.15 \mathrm{~mL}$ PFP solution containing $\mathrm{PbS}$ nanoparticles, and $0.03 \mathrm{~mL}$ anionic fluorosurfactant (Zonyl FSP, Sigma-Aldrich, USA) using emulsification with $10 \mu \mathrm{m}$ pore polymer membranes (Whatman, USA), following coarse emulsification by vortexing. PbS nanoparticles were imaged using a Hitachi HD-2000 scanning transmission electron microscope (TEM). The absorption coefficient of PbS-loaded PFP was determined using a Cary 6000i UV-vis-NIR spectrophotometer. The droplet diameters were measured using a microscope with an optical scale.

\subsubsection{Photoacoustic measurements}

A SASAM photoacoustic microscope (Kibero $\mathrm{GmbH}$, Germany) was used for all measurements and is described in detail in reference [144]. Briefly, a $1064 \mathrm{~nm}$ laser was focused by a 10x objective ( 0.30 numerical aperture) onto the sample from below, and a 375 
$\mathrm{MHz}$ transducer $\left(60^{\circ}\right.$ aperture, $42 \%$ bandwidth) positioned above the sample recorded the photoacoustic signals generated by the sample. The laser was operated at a $2 \mathrm{kHz}$ pulse repetition frequency with a 700 ps pulse width. This pulse width was less than the stress and thermal confinement times of a $1 \mu \mathrm{m}$ diameter liquid PFC droplet, calculated to be $2 \mathrm{~ns}$ and 8 $\mu$ s, respectively. The signals were amplified by $40 \mathrm{~dB}$ (Miteq, USA) and digitized at an 8 $\mathrm{GHz}$ rate $\left(0.125\right.$ samples/ns). All measurements were made at $36^{\circ} \mathrm{C}$. The droplet diameter was found by comparing a calibrated scale to an optical image recorded using the 10x objective. The error associated with this measurement is $\pm 0.5 \mu \mathrm{m}$ when using a $10 \mathrm{x}$ objective.

The photoacoustic signals from micron-sized nanoparticle-loaded droplets were measured in two different configurations; a droplet in suspension surrounded by liquid and a droplet on a hard boundary (figure 1). In case \#1, a droplet deposited on top of an agar gel surrounded by water was used to approximate a droplet in suspension. The agar gel has similar properties as water [164] and minimized any reflections that might occur. In case \#2, the droplet was deposited directly on the glass substrate, a highly reflecting surface for ultrasound. All measurements averaged 100 RF-lines to increase the signal to noise ratio (SNR). The transducer response was removed from the signal by normalizing the signal as described in [118] (see section 1.5), then a Hamming window and 100-500 MHz bandpass filter were applied. These processed signals were then compared to the analytical model and FEM solution. 


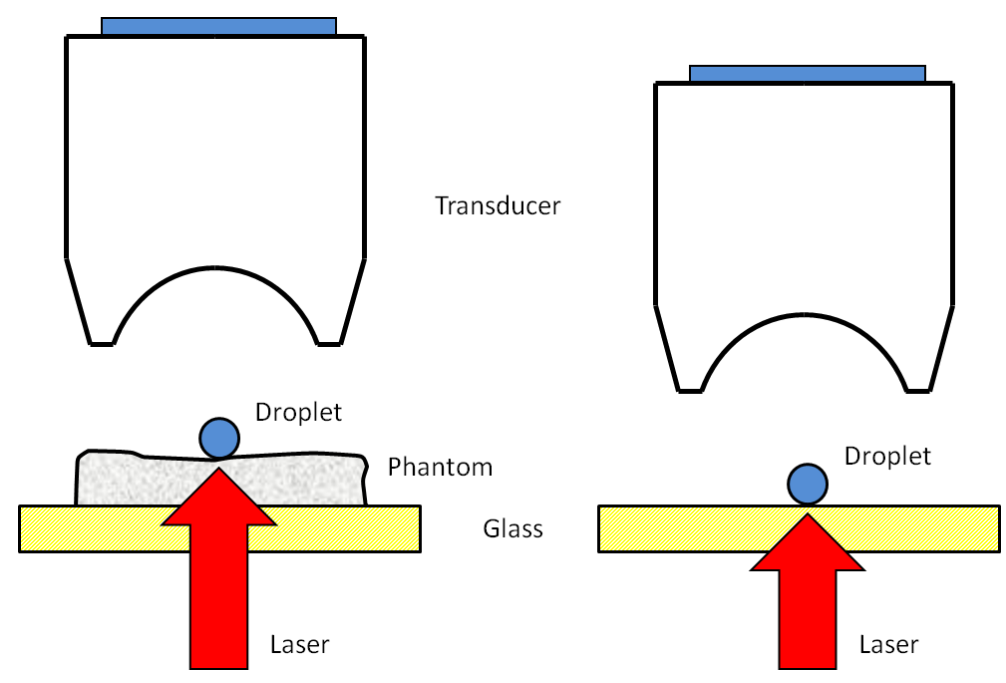

Figure 2.1: Diagram showing the two droplet configurations measured. The transducer positioned above the droplet recorded the photoacoustic signals from the droplet when irradiated by a $1064 \mathrm{~nm}$ laser from below. The photoacoustic signals from a droplet with a soft boundary (droplet on an agar surface, left) and a hard boundary (droplet directly on a glass substrate) were measured.

\subsubsection{COMSOL model}

Finite element models for each scenario were solved using the COMSOL Multiphysics software package. A transient acoustics 2D axisymmetric model was used to calculate the photoacoustic emission from the droplets using the wave equation (equation 11). For this calculation, the droplet diameter must be known, however the error associated with this optical image measurement is $\pm 0.5 \mu \mathrm{m}$ for droplets $<5 \mu \mathrm{m}$ in diameter. Since the exact diameter is not known, simulations were completed with a range of diameters around the measured diameter. The simulation with the most accurate photoacoustic spectral comparison to the experimental model was compared to the measured signal. Five droplets were examined in this study; one droplet in suspension (more droplets in suspension made 
with gold nanorods instead of $\mathrm{PbS}$ are examined in chapter 3), and four droplets on a glass substrate. The initial pressure was set to unity inside the droplet, and zero elsewhere. For scenario \#3, a droplet with an absorbing shell was simulated where the pressure within the shell of thickness $250 \mathrm{~nm}$ was set to unity, and zero elsewhere including the droplet core. For simplicity, these simulations assumed the shell was composed of PFC liquid, the same as the droplet core. The laser energy was assumed to be absorbed instantaneously (infinitesimally narrow laser pulse) so that the pressure wave did not propagate prior to the start of the simulation. The mesh within the droplet was set to $0.01 \mu \mathrm{m}$, and $0.05 \mu \mathrm{m}$ outside the droplet. and the outer boundary was set to a $10 \mu \mathrm{m}$ radius. The photoacoustic signal directly above the droplet was recorded as a function of time with a $0.125 \mathrm{~ns}$ step size (the same as the experimental rate). Diagrams of the three modeling scenarios are depicted in figure 2 . The FEM simulated a propagation time of $80 \mathrm{~ns}$, which took approximately 3 to 5 hours for 800,000 degrees of freedom using computational cluster containing six dual-core Opteron nodes and 40 GB of memory. The parameters used in the model are listed in table 1, including the diameter measured optically and the final diameter determined by a comparison between measured and simulated photoacoustic signals.

The PFP liquid inside the droplets exists in a superheated state; the boiling point of $\mathrm{PFP}$ is $29^{\circ} \mathrm{C}$, but remains a liquid at $36^{\circ} \mathrm{C}$ due to an increase in Laplace pressure caused by the shell; this increases the boiling point by $40-50^{\circ} \mathrm{C}$ [165]. Therefore it is difficult to measure the properties of bulk liquid PFP at temperatures higher than $29^{\circ} \mathrm{C}$. Studies measured the sound speed of PFP to be $468 \mathrm{~m} / \mathrm{s}$ using $2 \mathrm{MHz}$ at $22^{\circ} \mathrm{C}$ [166] and $477 \mathrm{~m} / \mathrm{s}$ 
using $1 \mathrm{MHz}$ at $25^{\circ} \mathrm{C}$ [167]. Measurements of several PFC liquids other than PFP showed a linear decrease in sound speed with increasing temperature from 25 to $36^{\circ} \mathrm{C}$ using $10 \mathrm{MHz}$ [168]. The change in sound speed was $-2.778 \mathrm{~m} / \mathrm{s} /{ }^{\circ} \mathrm{C}$ for $\mathrm{FC}-72$, the liquid with the closest molecular weight and structure to PFP. Assuming the change in sound speed with temperature of PFP is similar to the FC-72 even when in a superheated state, the sound speeds of $468 \mathrm{~m} / \mathrm{s}\left(22^{\circ} \mathrm{C}\right)$ and $477 \mathrm{~m} / \mathrm{s}\left(25^{\circ} \mathrm{C}\right)$ would be $429 \mathrm{~m} / \mathrm{s}$ and $446 \mathrm{~m} / \mathrm{s}$ at $36^{\circ} \mathrm{C}$, respectively. Averaging these values gives $438 \mathrm{~m} / \mathrm{s}$, which was used in the FEM and analytical simulations. Sound dispersion was neglected as it was found that PFC liquids have only a $1.5 \%$ increase from 100 to $1000 \mathrm{MHz}$ [169] and considered negligible over the frequency range used in this study.
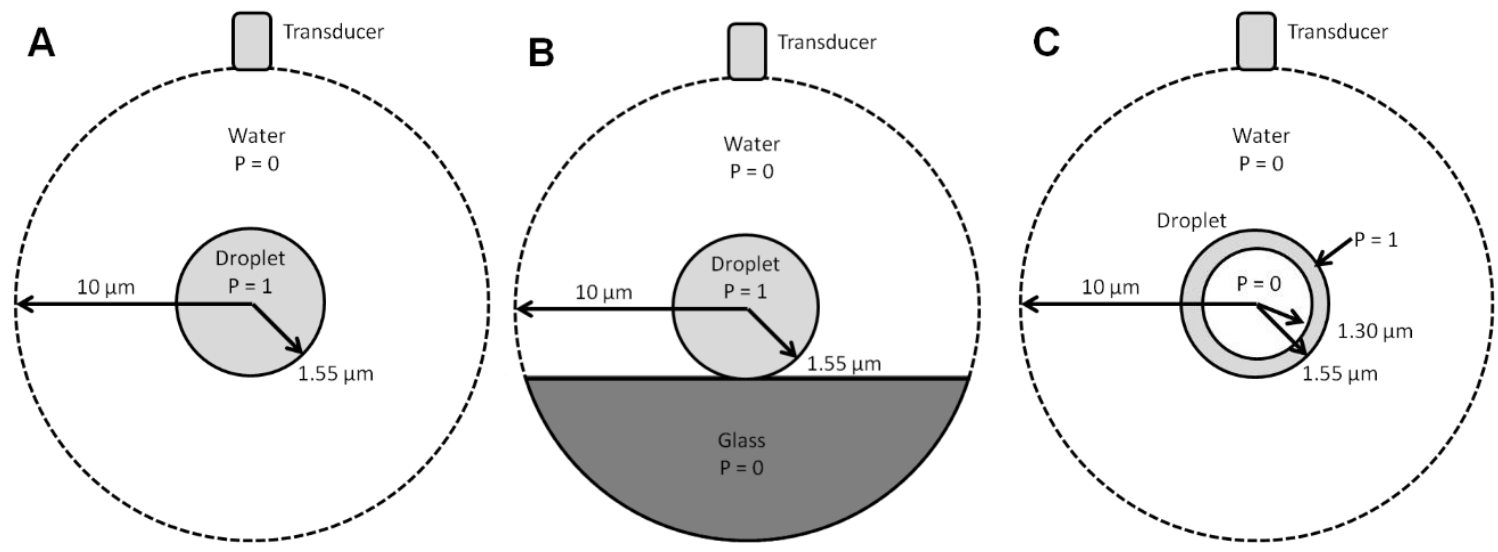

Figure 2.2: COMSOL model geometries used in this study. (A) a $3.1 \mu \mathrm{m}$ droplet in a water suspension, (B) a droplet on glass (diameters ranged from 2.8 to $3.6 \mu \mathrm{m}$ ) and (C) a $3.1 \mu \mathrm{m}$ droplet in a water suspension but with an optically absorbing $250 \mathrm{~nm}$ shell surrounding a non-absorbing core. In (A) and (B), the pressure inside the droplet was set to unity, and the surround water the pressure was set to zero. In (C), the pressure of the ring was set to unity, and the core was set to zero. The signal was recorded directly above the droplet. 
Table 2-1. Modeling parameters used in the finite element method models.

\begin{tabular}{ccccccc}
\hline Parameter & Droplet & Droplet & Droplet & Droplet & Droplet & Droplet \\
& $\# 1$ & $\# 2$ & $\# 3$ & $\# 4$ & $\# 5$ & $\# 6$ \\
\hline Simulation type & Suspension & Substrate & Substrate & Substrate & Substrate & Ring \\
Substrate & None & Glass & Glass & Glass & Glass & None \\
Optical diameter $(\mu \mathrm{m})$ & 3.4 & 3.1 & 4.0 & 4.2 & 4.4 & - \\
Droplet diameter $(\mu \mathrm{m})$ & 3.1 & 2.8 & 3.1 & 3.6 & 3.6 & 3.1 \\
System diameter $(\mu \mathrm{m})$ & 20 & 20 & 20 & 20 & 20 & 20 \\
Droplet mesh $(\mu \mathrm{m})$ & 0.01 & 0.01 & 0.01 & 0.01 & 0.01 & 0.01 \\
Coupling fluid mesh & & & & & & \\
$(\mu \mathrm{m})$ & 0.05 & 0.05 & 0.05 & 0.05 & 0.05 & 0.05 \\
Droplet sound speed & & & & & & 438 \\
$(\mathrm{~m} / \mathrm{s})$ & 438 & 438 & 438 & 438 & 438 & 1620 \\
Droplet density $\left(\mathrm{kg} / \mathrm{m}^{3}\right)$ & 1620 & 1620 & 1620 & 1620 & 1620 & 1620 \\
\hline
\end{tabular}

\subsection{Results}

\subsubsection{Finite element model validation}

The FEM solution of two PFP droplets $(3.1 \mu \mathrm{m}$ and $5.0 \mu \mathrm{m})$ suspended in water (figure 2A) were calculated using COMSOL Multiphysics with the parameters described in table 1 . The photoacoustic signal was calculated as a function of time at a point on the outer system boundary over an $80 \mathrm{~ns}$ time length. The FEM solution was compared to the analytical solution (equation 2-2) using identical parameters. The signals were normalized to maximum amplitude, and no other signal processing methods or filters were applied. Good agreement in both the time and frequency domains were observed as shown in Figure 2.3, 
thus validating the FEM methodology.

\subsubsection{Model and experiment comparison}

The photoacoustic signals of droplets on an agar surface and on a glass substrate were measured using the photoacoustic microscope. These two systems approximate a droplet in suspension and a droplet near a highly reflecting boundary. The measured signals were compared to both the analytical solution (equation 2-2) and the FEM solution. The parameters used for modeling the droplet in each case were identical aside from a difference in diameter. The theoretical time domain signal and power spectrum for a $3.1 \mu \mathrm{m}$ droplet using the analytical solution and FEM are shown in Figure 2.4. Both scenarios show spectral minima at 200, 345, 490 and $635 \mathrm{MHz}$, however the spectrum from the droplet on glass has additional spectral minima located at 275, 405 and $540 \mathrm{MHz}$.

The measured time domain signal for a $3.1 \mu \mathrm{m}$ droplet on agar (simulating a droplet in suspension) and a $3.1 \mu \mathrm{m}$ droplet on the glass substrate are shown in Figure $2.5 \mathrm{~A}$ and Figure 2.5B, respectively. Despite similar droplet sizes, the signal from the droplet on a glass boundary exhibits additional signal information. This is a result of the pressure wave rebounding from the glass substrate and towards to transducer, which can alter the frequency content. The corresponding power spectrum of these two droplets, along with three other droplets measured from a glass substrate are shown in Figure 2.6. In Figure 2.6A (droplet in suspension) the frequencies of the spectral minima at 203, 347 and $492 \mathrm{MHz}$ of the FEM and analytical solution are in good agreement with the measured signal from the droplet (spectral minima located at 205, 348 and $481 \mathrm{MHz}$. For the droplets on glass, the analytical solution 
could correctly predict the frequency of some of the spectral minima, but not all. Table 2-2 lists the frequencies of the spectral minima from the measured signal as well as from the analytical and FEM solutions. In all cases, the average standard deviation between the FEM and measured values were under $9 \mathrm{MHz}$.
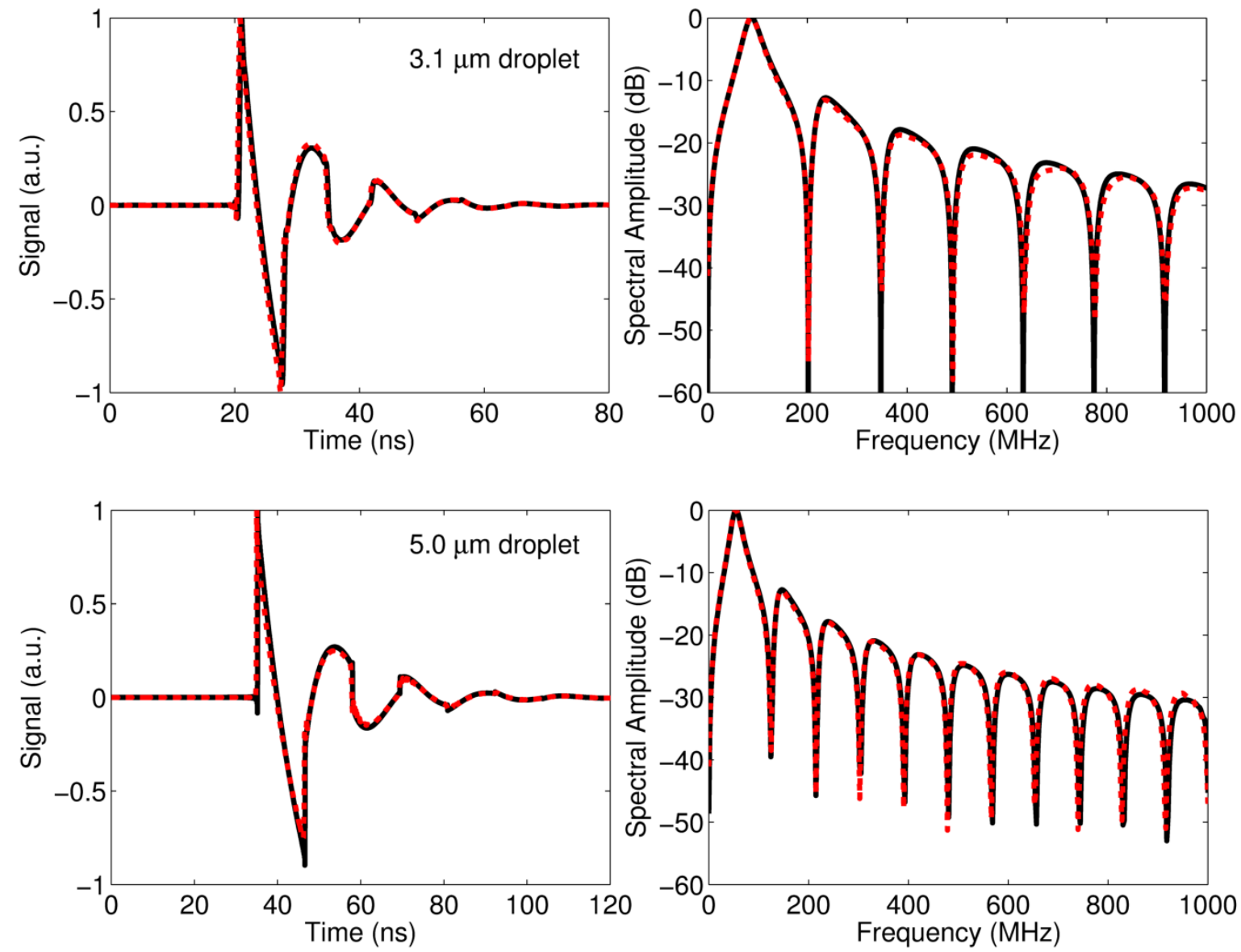

Figure 2.3: Theoretical waveform and spectrum $3.1 \mu \mathrm{m}$ droplet (top) and a $5.0 \mu \mathrm{m}$ droplet (bottom) suspended in water obtained from the analytical solution (equation 2-2, solid black line) and the FEM solution (dotted red line). The signals were normalized to maximum amplitude for comparison. Good agreement in both the time and frequency domains were observed, verifying the FEM methodology. 

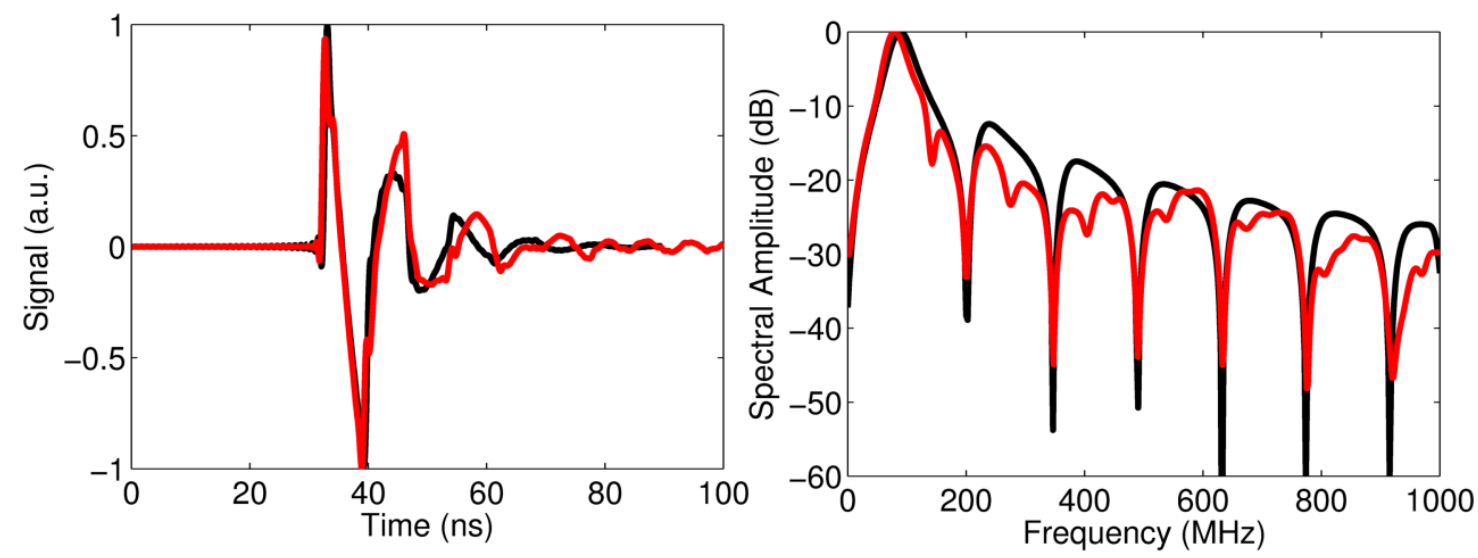

Figure 2.4: A comparison of the FEM solution of a $3.1 \mu \mathrm{m}$ droplet on glass (red) and the analytical solution of the same droplet in suspension (black). The signals were normalized to maximum amplitude for comparison. Small changes in the time domain signal result in extra spectral minima.
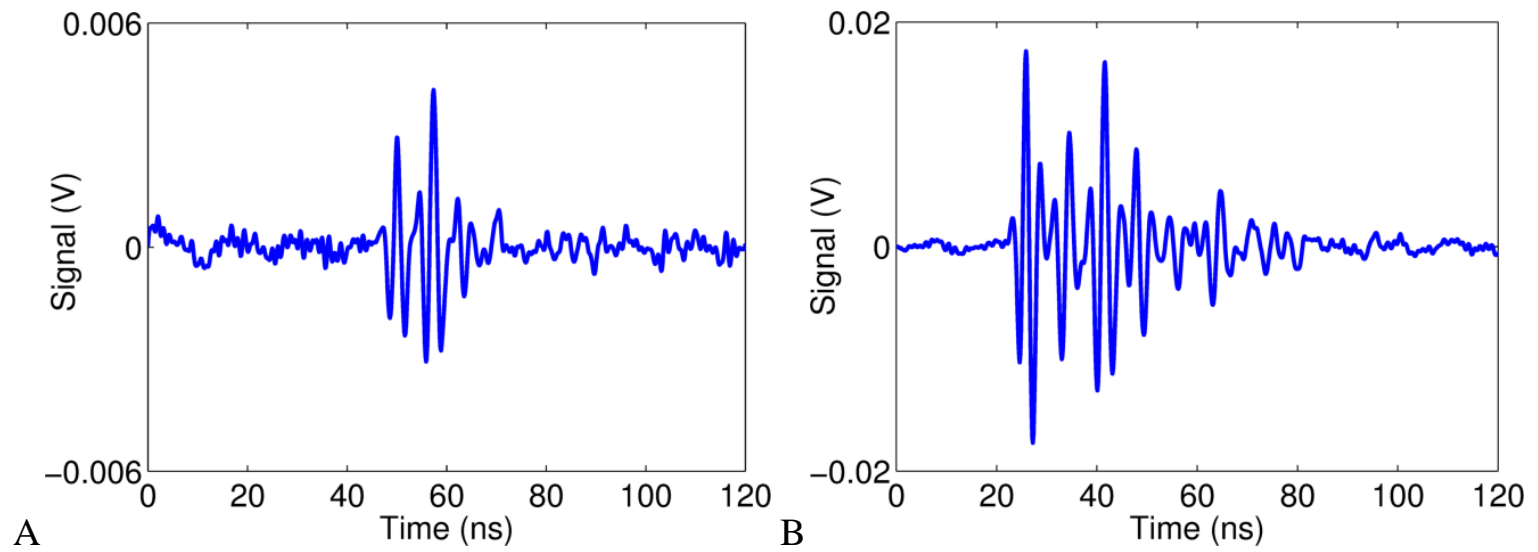

Figure 2.5: A representative measured photoacoustic signal from (A) $3.1 \mu \mathrm{m}$ droplet in suspension, and (B) a different $3.1 \mu \mathrm{m}$ droplet on a glass substrate. 

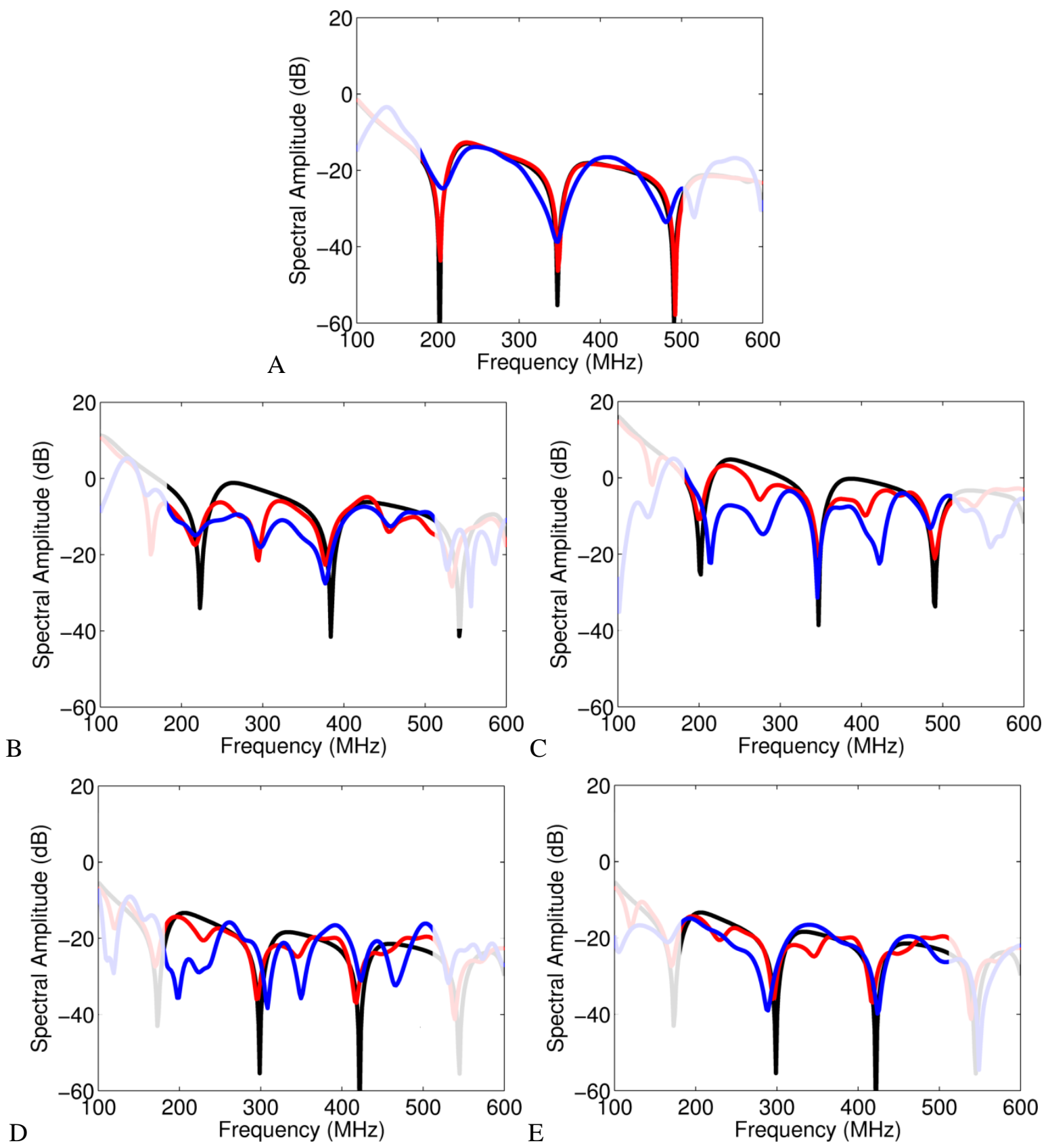

Figure 2.6: The photoacoustic spectrum from (A) a $3.1 \mu \mathrm{m}$ droplet on an agar surface (B-E) a droplet on glass with diameters $2.8,3.1,3.6$ and $3.6 \mu \mathrm{m}$ in diameter, respectively. The measured signals are blue, the analytical solution is black and the FEM solution is red. Good agreement in the location of the spectral minima measured and modeled solutions were observed for the droplet on an agar surface. For the droplet on a glass substrate, good agreement was observed between measured and the 
FEM solution only. The spectral region outside the transducer $-12 \mathrm{~dB}$ bandwidth of approximately $190-540 \mathrm{MHz}$ is noise and has been shaded to reduce attention to this area.

\begin{tabular}{|c|c|c|c|c|}
\hline Droplet & $\begin{array}{c}\text { Analytical } \\
\text { Model }(\mathrm{MHz})\end{array}$ & FEM (MHz) & Measured (MHz) & $\begin{array}{c}\text { Standard } \\
\text { Deviation }\end{array}$ \\
\hline 1 & $203,347,492$ & $203,347,492$ & $205,348,481$ & $3.3 \mathrm{MHz}$ \\
\hline 2 & $218,385,544$ & $219,295,377,457,533$ & $217,295,379,457,527$ & $1.4 \mathrm{MHz}$ \\
\hline 3 & $202,347,491$ & $201,277,346,406,490$ & $\begin{array}{r}215,279,346,422,484, \\
527\end{array}$ & $6.1 \mathrm{MHz}$ \\
\hline 4 & $\begin{array}{c}173,299,422, \\
545\end{array}$ & $170,230,297,347,416$ & $\begin{array}{r}197,228,309,350,426, \\
467\end{array}$ & $7.6 \mathrm{MHz}$ \\
\hline 5 & $\begin{array}{c}173,299,422, \\
545\end{array}$ & $170,230,297,347,416$ & $\begin{array}{r}166,246,289,371,424, \\
500\end{array}$ & $8.5 \mathrm{MHz}$ \\
\hline
\end{tabular}

Table 2-2: Spectral minima frequencies of the five droplets in Figure 2.6. The spectral minima associated with the glass substrate and not observed using the analytical model are coloured red. The last column is the average standard deviation between the frequency minima of the FEM and measured spectrum.

\subsubsection{Droplet surrounded by an absorbing ring}

The case of an optical absorbing ring surrounding a non-absorbing liquid core as shown in Figure 2.7 was examined. No analytical solution exists for this configuration. Contrast agents with this configuration have been developed and may be of interest to researchers, such as PLGA particles [170, 171], liposomes [172], porphysomes [158, 159] and similar structures. In the FEM, the pressure in the ring was set to unity, with the core and surrounding fluid pressure set to zero. All other parameters were the same as scenario \#1 (see table 1). The location of the spectra minima and maxima shifted as compared to the analytical solution that assumes a homogeneous uniformly absorbing sphere (figure 6). The 
distance between the spectral minima increased slightly with increasing frequency, while the distance was constant for the analytical solution. The shift in the location of the spectral peaks may be sufficient to differentiate an absorbing particle vs. an absorbing shell, however, it is unlikely the shell thickness could be estimated using this procedure.

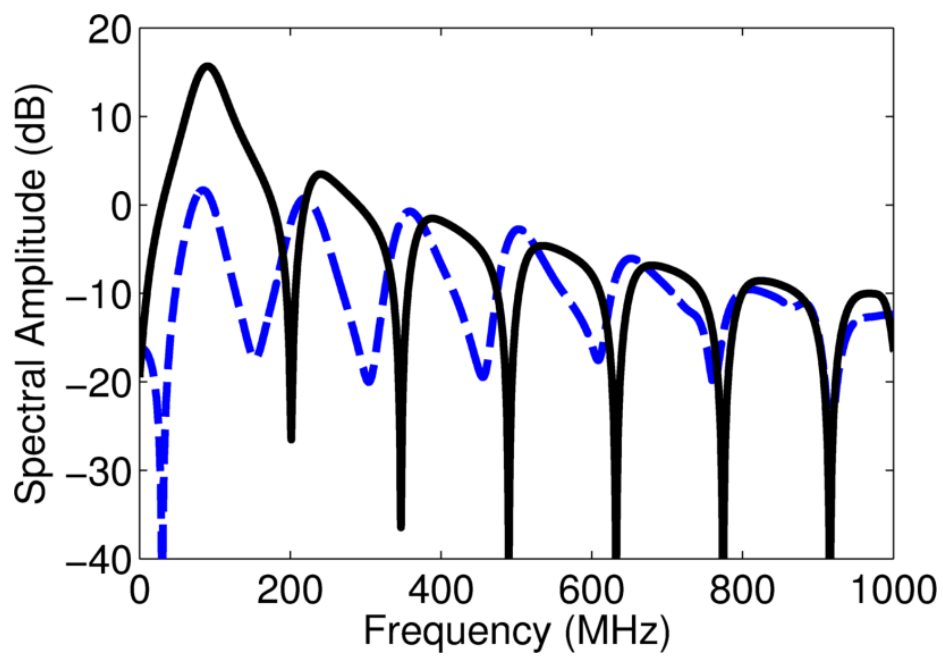

Figure 2.7: The theoretical photoacoustic spectrum from a $3.1 \mu \mathrm{m}$ droplet in suspension with an optically absorbing ring $250 \mathrm{~nm}$ thick surrounded a non-absorbing center (dotted blue line) compared to the analytical solution of a homogeneously absorbing liquid sphere in black.

\subsubsection{Visualization of the propagating signal}

Videos generated from the FEM simulation show the pressure wave expansion from a droplet in the three scenarios: a droplet in suspension, a droplet on a glass substrate and a droplet in suspension with a shell (see supplementary data). These videos enable visualization of the acoustic wave propagation through different materials. In all three scenarios, a positive pressure wave is shown propagating away from the droplet surface, with 
a negative pressure wave traveling toward the center. The low pressure wave then travels outward away from the droplet. This is responsible for the characteristic " $\mathrm{N}$ " shape typically observed in photoacoustic measurements, which is analogous to the pressure distribution observed during a bursting balloon [173]. For the droplet on the glass substrate, the acoustic wave travels faster through the glass than the coupling fluid, due to the difference in sound speed $(5600 \mathrm{~m} / \mathrm{s}$ in glass compared to $1520 \mathrm{~m} / \mathrm{s}$ in water). In the case of the absorbing ring (scenario 3), a narrow high pressure acoustic wave is observed travelling away from the droplet, with a narrow low pressure wave following a short time after.

\subsubsection{Discussion}

This study shows that spectral features calculated using the FEM simulation results are in good agreement with the analytical model for simple geometries such as a homogenous droplet in suspension (Figure 2.3), and gives accurate solutions where the analytical model breaks down, such as a droplet positioned on a hard reflecting boundary (Figure 2.7). For droplets on glass, the substrate reflects the pressure waves back through the droplet which are then recorded by the transducer. These additional waves result in additional spectral minima not observed from droplets in suspension. Table 2-2 summarizes the frequency of the spectral minima for all droplets, where the average standard deviation of the frequency of the spectral minima between the FEM and measured was less than $9 \mathrm{MHz}$.

Other photoacoustic FEMs take into account photon diffusion, heat transfer and stress/strain relations to calculate the pressure generated within an absorbing target after optical irradiation. Our primary interest was the frequency content of the photoacoustic 
waves; we have shown that simplifying the FEM by initiating the object at a pressure larger than the surroundings and using the wave equation to calculate the acoustic wave propagation is sufficient to calculate the signal generated and capture the frequencydependent spectral features. This simplified system decreases the model complexity and allows for faster computation and model development. The FEM could be applied to various scenarios where the analytical solution cannot be used, such as the generation of photoacoustic waves from multiple sources, heterogeneous absorbance and from asymmetric shapes such as the bi-concave shape of red blood cells where the photoacoustic spectral features are known to depend on RBC morphology [174].

The amplitude parameters in equation 2-2 can be adjusted to optimize the photoacoustic signal generation from these droplets to a limited extent. Increasing the laser intensity $I_{0}$ is not necessarily feasible as there are limits on the intensities that can be used. The sound speed, thermal expansion coefficient and heat capacity are inherent to the PFC liquid used with small variation between PFC liquids. The easiest way to increase the photoacoustic signal is to increase the absorption coefficient inside the droplet (by increasing the number of nanoparticles or their absorption coefficient), or increase the droplet size. This would increase the total absorption coefficient of the droplet, ultimately increasing the signal amplitude. Moreover, the larger droplets offer opportunities to carry a larger therapeutic payload to be activated at directly at a tumor site.

High frequencies were used in this study, as the materials studied had unique spectral features over the wide ultrasound bandwidth used (100-500 MHz). These spectral features 
can be used to extract specific information about the particle, such as their size and sound speed which can then be used for particle identification [175]. These high frequencies cannot be used in-vivo due to high attenuation of ultrasound, but could be used to identify cells and/or particles in a samples using the photoacoustic spectrum. Understanding how the photoacoustic signal is generated by these particles and propagates in a medium assists in development and optimization of these particles for eventual use in-vivo using clinical ultrasound and photoacoustic instruments.

\subsection{Conclusion}

Photoacoustic FEMs that incorporate photon transport, heat transfer, stress/strain relations and acoustic propagation into the solution are computationally complex. A simplified FEM using only the size and acoustic propagation parameters (sound speed and density) and an initial starting pressure within the object can be used to calculate the photoacoustic pressure waves emitted from an object during optical irradiation. This method simplifies the FEM complexity and reduces computational time, and generates the spectral features that are unique to the properties of the particles examined. The FEM can be applied towards complex configurations such as a droplet near a boundary or inhomogeneous particles to determine the particle-dependent spectral features. The FEM can easily be adapted to other complex systems such as multi-particles, asymmetric particles such as RBCs, heterogeneous absorption properties and boundary conditions where the analytical solution cannot be used. 


\subsection{Acknowledgements}

E. Strohm is supported through a NSERC doctoral scholarship. C. Kumaradas is acknowledged for his assistance in using the Ryerson computational cluster. This research was undertaken, in part, thanks to funding from NSERC and the Canada Research Chairs Program awarded to M. Kolios. Funding to purchase the equipment was provided by the Canada Foundation for Innovation, the Ontario Ministry of Research and Innovation, and Ryerson University. This study was supported, in part, by the Ontario Institute for Cancer Research Network through funding provided by the Province of Ontario, the FY07 Department of Defense Breast Cancer Research Program Concept Award (BC075873), a program project grant entitled "Imaging for Cancer" from the Terry Fox Foundation, and the Ontario Research Fund-Research for Excellence Program. 


\section{Chapter 3}

\section{Acoustic and photoacoustic characterization}

\section{of micron-sized perfluorocarbon emulsions ${ }^{1}$}

\subsection{Abstract}

Perfluorocarbon droplets containing nanoparticles (NPs) have recently been investigated as theranostic and dual-mode contrast agents. These droplets can be vaporized via laser irradiation or used as photoacoustic contrast agents below the vaporization threshold. This study investigates the photoacoustic mechanism of NP-loaded droplets using photoacoustic frequencies between 100 and $1000 \mathrm{MHz}$, where distinct spectral features are observed that are related to the droplet composition. The measured photoacoustic spectrum from NP-loaded perfluorocarbon droplets was compared to a theoretical model that assumes a homogenous liquid. Good agreement in the location of the spectral features were observed, which suggests the NPs act primarily as optical absorbers to induce thermal expansion of the

\footnotetext{
${ }^{1}$ This chapter is based on an original article published as: E. M. Strohm, I. Gorelikov, N. Matsuura, and M. C. Kolios, "Acoustic and photoacoustic characterization of micron-sized perfluorocarbon emulsions," Journal of Biomedical Optics, vol. 17, no. 9, pp. 096016-1-9, Sep. 2012.
} 
droplet as a single homogenous object. The NP size and composition do not affect the photoacoustic spectrum; therefore, the photoacoustic signal can be maximized by optimizing the NP optical absorbing properties. To confirm the theoretical parameters in the model, photoacoustic, ultrasonic and optical methods were used to estimate the droplet diameter. Photoacoustic and ultrasonic methods agreed to within $1.4 \%$, while the optical measurement was $8.5 \%$ higher; this difference decreased with increasing droplet size. The small discrepancy may be attributed to the difficulty in observing the small droplets through the partially translucent phantom.

\subsection{Introduction}

For several decades, perfluorocarbon (PFC) liquids have been used in various biomedical applications. PFCs are biologically and chemically inert that make them appealing for use in medicine [176], and they can be emulsified into nano- or micro-sized droplets [177] to overcome their insolubility in water for in vivo infusion. In 2000, it was reported that a liquid to gas phase change could be induced in PFC emulsions via ultrasound irradiation using a method called acoustic droplet vaporization (ADV) [165, 178]. The resulting bubbles could be used as ultrasound contrast agents [165, 179-181] and for cancer therapy via vessel occlusion [182] and enhance thermal ablation with high intensity focused ultrasound (HIFU) [183]. Furthermore, upon vaporization, chemotherapeutic agents within the emulsions can be released to deliver drugs to a target region [147, 184]. Recently, PFC

emulsions have been developed as photoacoustic agents through the incorporation of 
optically absorbing nanoparticles (NPs) $[144,145,148,185]$ or dyes [186] into the droplet. NP-loaded PFC droplets can be vaporized via light using a method called optical droplet vaporization (ODV) $[144,148]$ with similar applications as in ADV. Alternatively, the droplets could be irradiated below their vaporization threshold where they remain as liquid particles and used as photoacoustic contrast agents [144, 145].

NPs in the 10-200 nm range have been used as photoacoustic contrast agents in-vivo $[141,187,188]$ where they circulate within the bloodstream and extravasate into tumors via the enhanced permeability and retention effect (EPR) [189]. Using PFC droplets containing NPs as photoacoustic contrast agents may provide advantages over using NPs alone. The NPs within a droplet are concentrated into a small volume, so fewer NPs may be required to create a measurable photoacoustic signal compared to free NPs. The photoacoustic signal from NPs is dependent on the environment surrounding the NP [190]. Enclosing the NPs within a droplet of known liquid allows for direct optimization of the NPs within the liquid to maximize the photoacoustic signal output, whereas the signal from free NPs may differ depending on their location (such as blood, interstitial tissue or within single cells). It is also possible to functionalize the PFC droplet surface with targeting ligands or imaging agents [191]. The droplets can be loaded with therapeutic agents to deliver a targeted payload to a region of interest [147, 184]. Finally, the droplet size can be tailored for specific applications, ranging from nm-sized (for penetration into interstitial tissue) to micron-sized (restricted to the bloodstream). The droplets could be used as photoacoustic contrast agents to determine their location, and then vaporized to release a payload and/or the NPs into surrounding tissue. 
There have been very few studies examining the photoacoustic mechanism from NP-loaded droplets.

NPs are increasingly being used as photoacoustic contrast agents. This paper demonstrates that micron-sized PFH droplets loaded with optical absorbing, silica-coated gold NPs act as a single photoacoustic emitter when irradiated with the appropriate wavelengths, and can be used as photoacoustic contrast agents. The droplet photoacoustic spectral features, from droplets of various sizes, are investigated to show that the droplet photoacoustic spectrum features depend on the size and physical properties of the liquid within the droplet only. The NPs are only used to initiate the thermal expansion within the droplet. These findings indicate the NP-loaded droplets provide advantages over using NPs alone.

\subsection{Theory}

When irradiated by a laser with an appropriate wavelength, optically absorbing structures will absorb energy and undergo a rapid thermoelastic expansion, resulting in emission of a pressure wave that can be detected with conventional ultrasonic transducers. In this study, silica-coated gold NPs were selected as the optical absorbers, due to their strong absorption peak at the laser frequency used, and their ability to be loaded within the PFC droplets [144]. A well-established theoretical model was used to calculate the photoacoustic pressure waves generated from homogeneous optical absorbing liquid spheres [56]. Assuming the droplet undergoes a uniform thermal expansion due to the heated NPs, the 
frequency domain pressure wave generated a distance $r$ from a droplet with radius $a$ when irradiated with a laser intensity $I_{0}$ is

$$
P(f)=i\left(\frac{\mu_{a} I_{0} c_{d} \beta}{4 \pi C_{p}(r / a)}\right) \frac{(\sin q-q \cos q) / q^{2}}{\left(1-\frac{\rho_{d}}{\rho_{f}}\right)(\sin q / q)-\cos q+i \frac{c_{d}}{c_{f}} \frac{\rho_{d}}{\rho_{f}} \sin q},
$$

where $f$ is the ultrasound frequency, $\beta$ is the droplet thermal expansion coefficient, $C_{\mathrm{p}}$ is the droplet heat capacity, $\mu_{\mathrm{a}}$ is the optical absorption coefficient of the NPs within the droplet, $\rho$ is the density, $c$ is the sound speed, the subscripts $d$ and $f$ refer to the droplet and surrounding fluid, respectively, and $q=2 \pi f a / c_{\mathrm{d}}$ [56]. In this equation, the variables in the first bracket contribute only to the amplitude of the signal and can be represented by a constant $A$. The only variables that contribute significantly to spectral variations (the component to the right of the bracket in equation 3.1) are the droplet radius, density and sound speed. This equation also assumes that the physical parameters such as sound speed do not change with frequency. Although this assumption is valid for liquids where sound dispersion is absent over small frequency ranges, in this study a frequency range of 100-1000 MHz was used, where PFC liquids have a small but measureable increase in sound speed with frequency [169]. To account for this dispersion, equation 3.1 was modified by setting the droplet sound speed as a function of frequency, and setting the variables that contribute to the amplitude to a constant $A$, so that the pressure $P$ as a function of frequency is

$$
P(f)=i A \frac{(\sin q-q \cos q) / q^{2}}{\left(1-\frac{\rho_{d}}{\rho_{f}}\right)(\sin q / q)-\cos q+i \frac{c_{d}(f)}{c_{f}} \frac{\rho_{d}}{\rho_{f}} \sin q} \text { where } q=\frac{2 \pi f a}{c_{d}(f)} \text {. }
$$


The droplet radius, density and sound speed all contribute to the frequency content of the generated photoacoustic signal. If the PFC liquid type (e.g. perfluorohexane) is kept constant, then the droplet radius is the only parameter that affects the photoacoustic spectral features. The droplet diameter can be calculated by adjusting the radius in the model until a best fit between the model and measured photoacoustic spectrum is found. To confirm the accuracy of the diameter parameter in the model, the diameter can also be determined using photoacoustic and ultrasonic pulse echo methods based on the time of flight of the photoacoustic signals or reflected echoes from the droplet surfaces. A characteristic "Nshape" wave in the time domain is generated composed of a positive pressure followed by a negative pressure [55]. If the sound speed $c_{\mathrm{d}}$ in the droplet is known, the droplet diameter can be found from the arrival time of the pressures wave using

$$
d=c_{d}\left(t_{2}-t_{1}\right)
$$

where $t_{1}$ is time of flight of the positive pressure peak and $t_{2}$ is the time of flight of negative pressure peak. Similarly, when a droplet is probed with pulsed ultrasound, echoes will occur from the top and bottom of the droplet due to the acoustic impedance mismatch between the droplet and surrounding fluid. The diameter of the droplet can be found using

$$
d=\frac{c_{d}}{2}\left(t_{2}-t_{1}\right)
$$

where $t_{1}$ is the echo from the top of the droplet and $t_{2}$ is the echo from the bottom of the droplet [11]. In total, four independent methods were used to determine the PFC droplet 
diameters in this study.

In equation 3.2, it is essential to know how the sound speed varies as a function of frequency (known as sound dispersion). Previous work measuring the sound speed and attenuation of PFC liquids determined that the sound speed and ultrasound attenuation of perfluorohexane (PFH) increases with frequency [169]. The increase in attenuation $\alpha(f)$ with frequency is generally described using a power law fit, such as $\alpha(f)=\alpha_{0} f^{\mathrm{n}}$, where the attenuation coefficient $\alpha_{0}$ and the power law exponent $n$ describe how the attenuation changes with frequency [192]. A direct relationship between sound dispersion and ultrasonic attenuation can be accurately described using the Kramers-Kronig relation [193]

$$
c(f)=\left[\frac{1}{c_{d}}+\alpha_{0} \tan \left(\frac{n \pi}{2}\right)\left(|2 \pi f|^{n-1}-\left|2 \pi f_{0}\right|^{n-1}\right)\right]^{-1},
$$

where $\alpha_{0}=\alpha(\mathrm{f}) / \mathrm{f} \mathrm{n}$ is the attenuation coefficient (with units Nepers, converted to $\mathrm{dB}$ by multiplying by 8.686 ), and $\mathrm{f}_{0}$ is the frequency where cd was measured [194]. The change in sound speed with frequency can be determined using equation 3.5 if $\alpha_{0}$ and $\mathrm{n}$ are known for a specific liquid.

\subsection{Method}

\subsubsection{PFC emulsions}

Gold NPs were synthesized using a previously published method based on the reduction of sodium citrate [195]. Specifically, gold NPs were made by loading $100 \mathrm{mg}$ of sodium citrate and $150 \mathrm{~mL}$ of de-ionized water into a $250 \mathrm{~mL}$ round bottom flask, heating the 
mixture to boiling, followed by the injection of $1 \mathrm{~mL}$ of $25 \mathrm{mM} \mathrm{HAuCl}_{4}$. The temperature of the mixture was slowly decreased to $90^{\circ} \mathrm{C}$ over a 15 minute time period, and $1 \mathrm{~mL}$ of $60 \mathrm{mM}$ sodium citrate was then injected, followed by the injection of $0.9 \mathrm{~mL}$ of $25 \mathrm{mM} \mathrm{HAuCl} 4$. After 15 minutes, the injections of sodium citrate and $\mathrm{HAuCl}_{4}$ were repeated, resulting in the formation of gold NPs. To miscibilize gold NPs into perfluorohexanes (FC-72, Synquest Labs, FL, USA), the NPs were coated with silica [196], followed by surface fluorination $[163,197]$. Specifically, to coat the NPs with silica, a freshly prepared aqueous solution of 3aminopropyl trimethoxy silane (APTES, $1.6 \mathrm{~mL}, 1 \mathrm{mM}$ ) was added to $150 \mathrm{~mL}$ of the gold NPs solution under vigorous magnetic stirring. The APS and gold dispersion were reacted for 15 minutes to ensure complete complexation of the amine groups with the gold surface. A solution of active silica was prepared by lowering the $\mathrm{pH}$ of a $0.54 \mathrm{wt} \%$ sodium silicate solution to 11 by progressive additions of $1.0 \mathrm{M} \mathrm{HCl}$. Nine $\mathrm{mL}$ of active silica was then added to $150 \mathrm{~mL}$ of the surface modified gold NPs, again under vigorous magnetic stirring. The resulting dispersion $(\mathrm{pH}=8.5)$ was then allowed to stand for a minimum of 24 hours, to permit the active silica to polymerize onto the surface of the gold NPs. For miscibilization of gold NPs into PFH, the silica-coated gold NPs were redispersed in $10 \mathrm{~mL}$ of methanol [161], and the mixture was reacted with $150 \mu \mathrm{L}$ of $1 \mathrm{H}, 1 \mathrm{H}, 2 \mathrm{H}, 2 \mathrm{H}$-perfluorodecyltriethoxysilane (5 minutes), and mixed with $50 \mu \mathrm{L}$ of $30 \%$ ammonia/water and reacted for 24 hours. After evaporation of methanol, the NPs were solubilized into $2 \mathrm{~mL}$ of PFH. All chemicals for silica-coated gold NP synthesis and their miscibilization into PFCs $\left(\mathrm{HAuCl}_{4}\right.$, sodium citrate, APS, sodium silicate and $1 \mathrm{H}, 1 \mathrm{H}, 2 \mathrm{H}, 2 \mathrm{H}$-perfluorodecyltriehthoxysilane) were purchased 
from Sigma-Aldrich, ON, Canada. The thickness of the silica coating and the size of the gold NPs were determined by transmission electron microscopy using a Hitachi S-5200 scanning electron microscope (Hitachi Canada Ltd., ON, Canada). For NP characterization in the ultraviolet, visible, and near-IR ranges, a Cary-5000 UV-Vis-NIR spectrophotometer (Agilent Technologies, CA, USA) was used. Gold NP-incorporated PFH droplets were prepared using $5 \mathrm{~mL}$ of deionized water (Millipore Milli-Q grade, $18.2 \mathrm{M} \Omega$ ), $0.175 \mathrm{~mL}$ of gold NP-FC-72 solution, and $0.025 \mathrm{~mL}$ of anionic phosphate fluorosurfactant (Zonyl-FSP, Sigma Aldrich, ON, Canada). Droplets were prepared by coarse emulsification by vortexing, followed by membrane emulsification using $10 \mu \mathrm{m}$ pore size polycarbonate membranes (Nuclepore Track-Etch Membrane, Whatman, NJ, USA) [144].

$\mathrm{PFH}\left(56^{\circ} \mathrm{C}\right.$ boiling point) was used instead of the more commonly used perfluoropentane (PFP, $29^{\circ} \mathrm{C}$ boiling point) as micron-scale $\mathrm{PFH}$ droplets are more stable and are less likely to spontaneously vaporize at the temperatures used in these experiments compared to PFP droplets [198, 199]. Moreover, the sound speed of bulk PFP could not be measured at $36^{\circ} \mathrm{C}$ as it is over the boiling point of $29^{\circ} \mathrm{C}$ and it would be difficult to use the theoretical photoacoustic equations. The sound speed of bulk PFH has been previously measured at the frequencies and temperatures used [169].

\subsubsection{Photoacoustic microscope}

All acoustic and photoacoustic measurements were made using the SASAM acoustic microscope (Kibero GmbH, Germany). An IX81 inverted optical microscope (Olympus, Japan) was modified where a transducer was positioned above the optical objective. The 
sample is positioned between the optical objective and transducer, allowing for optical observation during measurements. Ultrasonic pulse echo measurements were made by scanning the sample under the transducer. For photoacoustic measurements, a $532 \mathrm{~nm}$ laser was collimated through the back port of the inverted microscope and focused onto the sample with the same objective used to view the sample. A dichroic mirror (Chroma Technology Corp, USA) was used to reflect optical wavelengths between $450-620 \mathrm{~nm}$, but pass all other wavelengths to allow for optical viewing and targeting when using the laser (figure 3.1A). The transducer was focused above the laser spot under optical guidance, and the sample was scanned under the transducer with both the optical objective and transducer remaining stationary (figure 3.1B).

Transducers with center frequencies of $375 \mathrm{MHz}\left(60^{\circ}\right.$ aperture, $42 \%$ bandwidth) and 750 $\mathrm{MHz}\left(100^{\circ}\right.$ aperture, $37 \%$ bandwidth) were used for all acoustic and photoacoustic measurements. The $750 \mathrm{MHz}$ transducer provided a wider bandwidth for analysis that is not possible with the $375 \mathrm{MHz}$ transducer. For acoustic measurements, a $10 \mathrm{~V}_{\mathrm{pp}}$ monocycle signal was generated at $300 \mathrm{MHz}$ (for the $375 \mathrm{MHz}$ transducer) and at $1000 \mathrm{MHz}$ (for the $750 \mathrm{MHz}$ transducer) at a pulse repetition frequency (PRF) of up to $500 \mathrm{kHz} .100$ signals were averaged at $375 \mathrm{MHz}$, while 1000 signals were averaged at $750 \mathrm{MHz}$. More averaging was used at $750 \mathrm{MHz}$ to increase SNR, as the measured signals are typically lower at higher frequencies. For photoacoustic measurements, the laser was focused onto the sample using a 10x optical objective ( 0.3 numerical aperture). The laser triggered the data acquisition of the signals the transducer recorded. The laser had a pulse width of $330 \mathrm{ps}$ and PRF of $4 \mathrm{kHz}$. All 
signals were amplified by a $40 \mathrm{~dB}$ amplifier (Miteq, USA) and digitized at $8 \mathrm{GHz}$. All measurements were made at $36^{\circ} \mathrm{C}$ to simulate human physiological conditions. Further details on the system can be found elsewhere [106, 144].

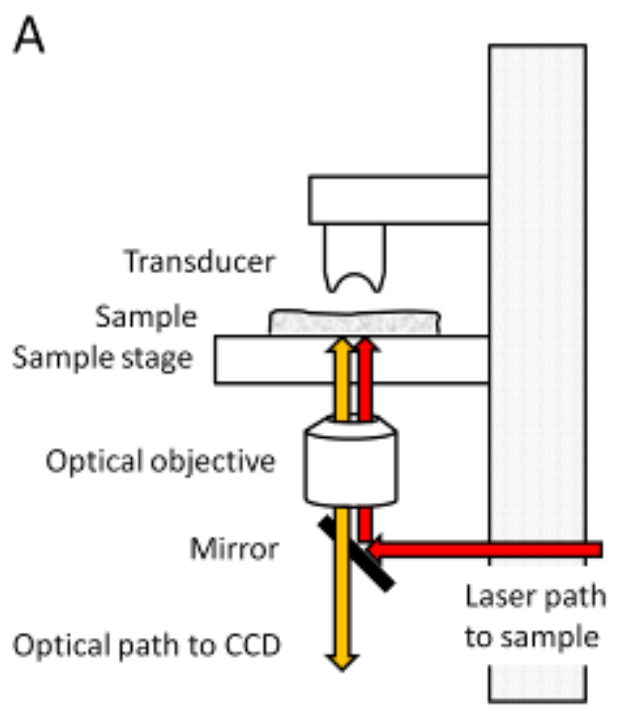

B

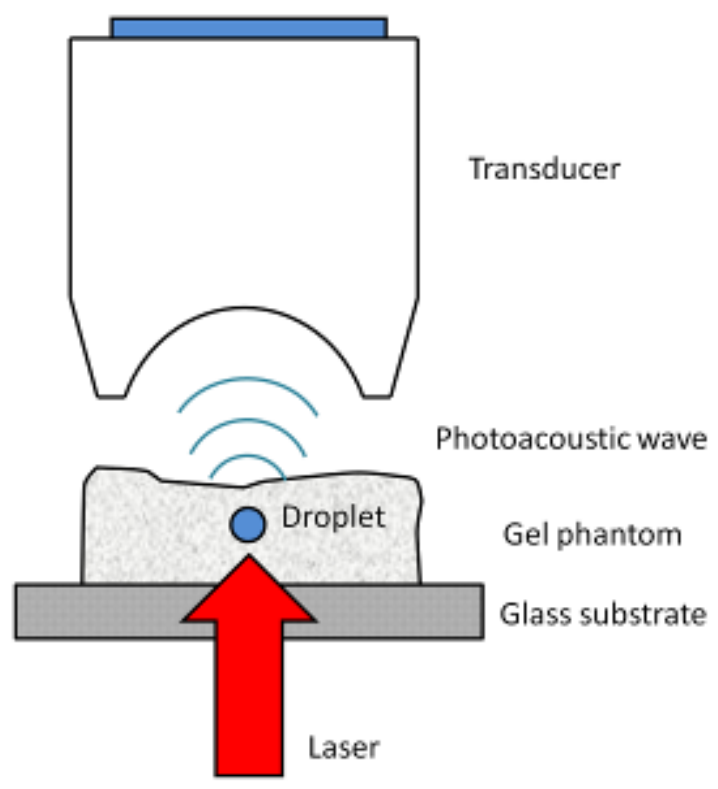

Figure 3.1: (A) The acoustic microscope is an inverted optical microscope where the laser has been focused through the pack port onto the sample. The mirror reflects 500-650 nm (the laser wavelengths) towards the sample, but allows other wavelengths to pass for optical viewing. The transducer measures the signals from above the sample. (B) A close-up view of a measurement of a single droplet. The laser is focused onto the sample using the optical objective with the transducer positioned directly above the droplet. 


\subsubsection{Acoustic and photoacoustic measurements}

Droplets were embedded into gelatin phantoms to ensure they were immobilized during the measurements. Droplets were added to liquid gelatin at a temperature of $37^{\circ} \mathrm{C}$, and then formaldehyde was added to increase phantom stiffness. Droplets close to the phantom surface were measured to minimize the attenuation losses through gelatin. All acoustic and photoacoustic measurements at $375 \mathrm{MHz}$ were made with the droplets embedded within the gelatin. No signal from embedded droplets was detected at $750 \mathrm{MHz}$ due to attenuation through the gelatin, therefore droplets were deposited onto the phantom surface for the 750 $\mathrm{MHz}$ photoacoustic measurements. Subsequent measurements using the $375 \mathrm{MHz}$ transducer were not possible, as the droplets would move from the field of view while switching transducers. The acoustic properties of gelatin and water are very similar [164], and the spectral features only depend on the sound speed and density of the droplet and coupling fluid in equation 3.2. Therefore it is unlikely the change in medium between similar liquids would significantly affect the spectral shape.

To record the acoustic and photoacoustic signals, a droplet was positioned over the laser spot and then the transducer was focused above the droplet. The sample stage was scanned over a $10 \times 10 \mu \mathrm{m}$ area using a $0.5 \mu \mathrm{m}$ step size, and the signal from the center of the droplet was used for analysis. The optical objective and transducer remained stationary and co-aligned during all scans. The transducer response was removed by normalizing the signal as described elsewhere [118]. Briefly, the normalized signal was calculated by dividing the measured spectrum of the droplet by the reference spectrum of the transducer. The bandwidth 
of a transducer is typically found by measuring the reflected echoes from a glass or quartz substrate that reflects the ultrasound back to the transducer. We have found that this is not an accurate representation of the transducer bandwidth used in photoacoustics since the transducer is used in passive mode. The transducers appear to have better sensitivity when receiving (photoacoustic) than during transmit and receive (ultrasound). A $200 \mathrm{~nm}$ gold layer theoretically has a flat photoacoustic spectrum to within $3 \mathrm{~dB}$ from 1 to $1000 \mathrm{MHz}$ [57], and will give an accurate representation of the transducer spectral response in passive mode; therefore, we used the photoacoustic signal from a gold film as a reference. A Hamming window was applied to all signals, along with a bandpass filter of $100-500 \mathrm{MHz}$ for the 375 MHz transducer and 300-1000 MHz for the $750 \mathrm{MHz}$ transducer.

\subsubsection{Droplet diameter measurements}

The droplet diameter was calculated using three independent methods: 1) the photoacoustic time domain signal, 2) the time domain ultrasound signal, and 3) fitting the measured photoacoustic spectrum to the model. Only the signals measured using the 375 $\mathrm{MHz}$ transducer were used in the diameter calculations. These calculations were compared to images recorded from a CCD camera using an optical microscope with a 10x objective (100x total magnification).

The photoacoustic method used the time of flight of the positive and negative pressure waves from the droplet and equation 3.3 to determine the droplet diameter. The pressure wave emitted from a droplet during optical irradiation has two significant features: an initial positive sharp pressure peak that gradually decreases to a negative pressure peak. 
This waveform has a characteristic "N-shape" in the time domain, analogous to pressure waves generated from a bursting balloon $[173,200]$. The theoretical time domain signal for a $5 \mu \mathrm{m}$ PFH droplet is shown in figure 3.2A (black dotted line), where the N-shape waveform is clearly visible. However, the finite bandwidth of a transducer can alter the signal. For example, the same signal subjected to a Hamming window and a 100-500 $\mathrm{MHz}$ bandpass filter (to emulate the limited transducer bandwidth) is shown in figure 3.2A (red solid line). Instead of an $\mathrm{N}$-shape, two peaks are visible which align with the positive and negative peaks of the N-shape waveform. These two peaks can be used to determine the droplet diameter using equation 3.3, where $t_{1}$ is the positive peak on the first signal and $t_{2}$ is the negative peak on the second signal.

The ultrasound pulse echo method used the time of flight of the echoes from the top and bottom of the droplet ( $t_{1}$ and $t_{2}$ in figure 3.2B) in equation 3.4 to determine the droplet diameter. This approach required that the droplet was sufficiently large so that the two echoes were separated in time and could be resolved. This method could not be used for droplets smaller than approximately $5 \mu \mathrm{m}$, as the signals would overlap.

The photoacoustic spectral method was used to calculate the diameter by comparing the spectrum from the measured photoacoustic signal to the model (equation 3.2). The model was calculated using a constant density of $1650 \mathrm{~kg} / \mathrm{m}^{3}$, and the sound speed was varied from 480 to $486 \mathrm{~m} / \mathrm{s}$ with increasing frequency from 100 to $1000 \mathrm{MHz}$ (equation 3.5). The diameter was adjusted until the minima and maxima between experiment and model matched. The diameter found from these signal processing methods were then compared to a 
direct optical measurement from the microscope.
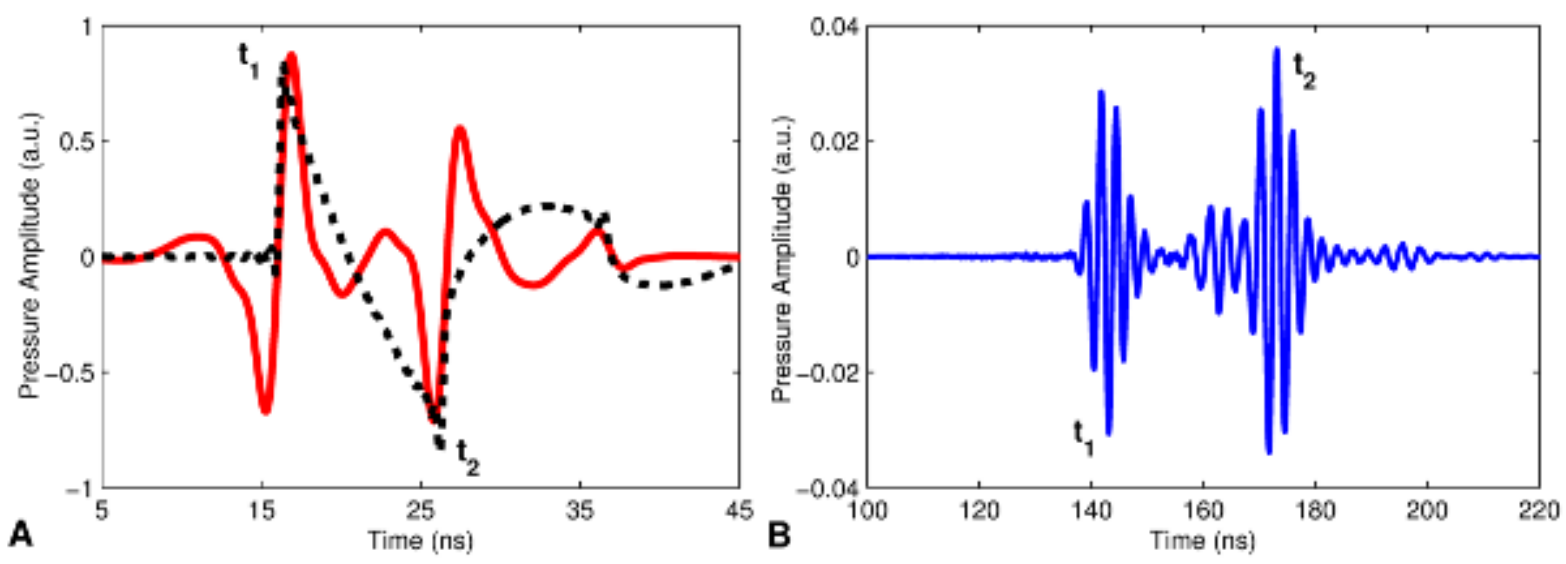

Figure 3.2: (A) The theoretical photoacoustic time domain signal from a $5 \mu \mathrm{m}$ droplet using equation 3.2 (black dotted line), and the same signal after applying a Hamming window and 100-500 $\mathrm{MHz}$ bandpass filter (red solid line). (B) The measured ultrasound echoes from a 7.2 $\mu \mathrm{m}$ PFH droplet embedded in gelatin. The signals labeled $t_{1}$ and $t_{2}$ were used to determine the droplet diameter using equation 3.3 (photoacoustic, left) and equation 3.4 (ultrasound, right).

\subsection{Results and discussion}

\subsubsection{Droplet configuration}

Silica-coated gold NPs were used as the optical absorbing NP in this study due to their strong optical absorption peak around $540 \mathrm{~nm}$. Transmission electron microscopy images demonstrated these NPs were of uniform size, with a $20 \mathrm{~nm}$ diameter core and $5 \mathrm{~nm}$ thick silica shell (figure 3.3A). The optical absorption of the PFH solution containing NPs peaked between $530-540 \mathrm{~nm}$ (figure 3.3B), an optimum range for the $532 \mathrm{~nm}$ pulsed laser used in this study. The laser energy for all photoacoustic measurements was $30 \mathrm{~nJ} / \mathrm{pulse}$, 
focused to a spot size approximately $10 \mu \mathrm{m}$ in diameter, resulting in a laser fluence of 38 $\mathrm{mJ} / \mathrm{cm}^{2}$. Although damage to gold NPs has been reported for laser fluences as low as 25 $\mathrm{mJ} / \mathrm{cm}^{2}$ at $532 \mathrm{~nm}$ [201], damage thresholds can depend on the gold NP surface and coating, and other laser parameters such as pulse length. In this study, the photoacoustic signals from the NP-loaded droplets (ranging in size from 2 to $15 \mu \mathrm{m}$ ) were stable over repeated measurements using laser fluences of $38 \mathrm{~mJ} / \mathrm{cm}^{2}$, indicating damage to NPs was negligible. At higher fluence levels (over $50 \mathrm{~mJ} / \mathrm{cm}^{2}$ ), the measured photoacoustic signal decreased rapidly over repeated pulses, which could be attributed to NP damage. The vaporization threshold for these droplets was between $50-120 \mathrm{~mJ} / \mathrm{cm}^{2}$, considerably less than $1.5-3 \mathrm{~J} / \mathrm{cm}^{2}$ required to vaporize PFH droplets containing lead sulphide NPs reported previously using a $1064 \mathrm{~nm}$ laser. This is likely due to the much higher absorption coefficient of the gold NPs $\left(110 \mathrm{~cm}^{-1}\right.$ at $\left.532 \mathrm{~nm}\right)$ compared to lead sulphide NPs $\left(4.3 \mathrm{~cm}^{-1}\right.$ at $\left.1064 \mathrm{~nm}\right)$.

The number of NPs inside the droplets was estimated based on the initial concentration of NPs in the PFC liquid $\left(\sim 10^{14} \mathrm{NPs} / \mathrm{mL}\right.$ of PFC). Assuming the NPs are distributed uniformly within the droplets, there are approximately 6550 NPs in $5 \mu \mathrm{m}$ droplets $\left(6.7 \times 10^{-11} \mathrm{cc}\right)$ and $52300 \mathrm{NPs}$ in $10 \mu \mathrm{m}$ droplets $\left(5.7 \times 10^{-10} \mathrm{cc}\right)$. The approximate distance from one NP to its neighbor can be calculated by estimating the volume fraction of spheres that can fill a single droplet using a random close packing model [202]. Based on this model, the average distance between NPs is $\sim 230 \mathrm{~nm}$. Although some non-uniform distribution of NPs within the droplets is possible, as an order of magnitude calculation, the calculated values for the NP quantity and NP-spacing seem reasonable. 

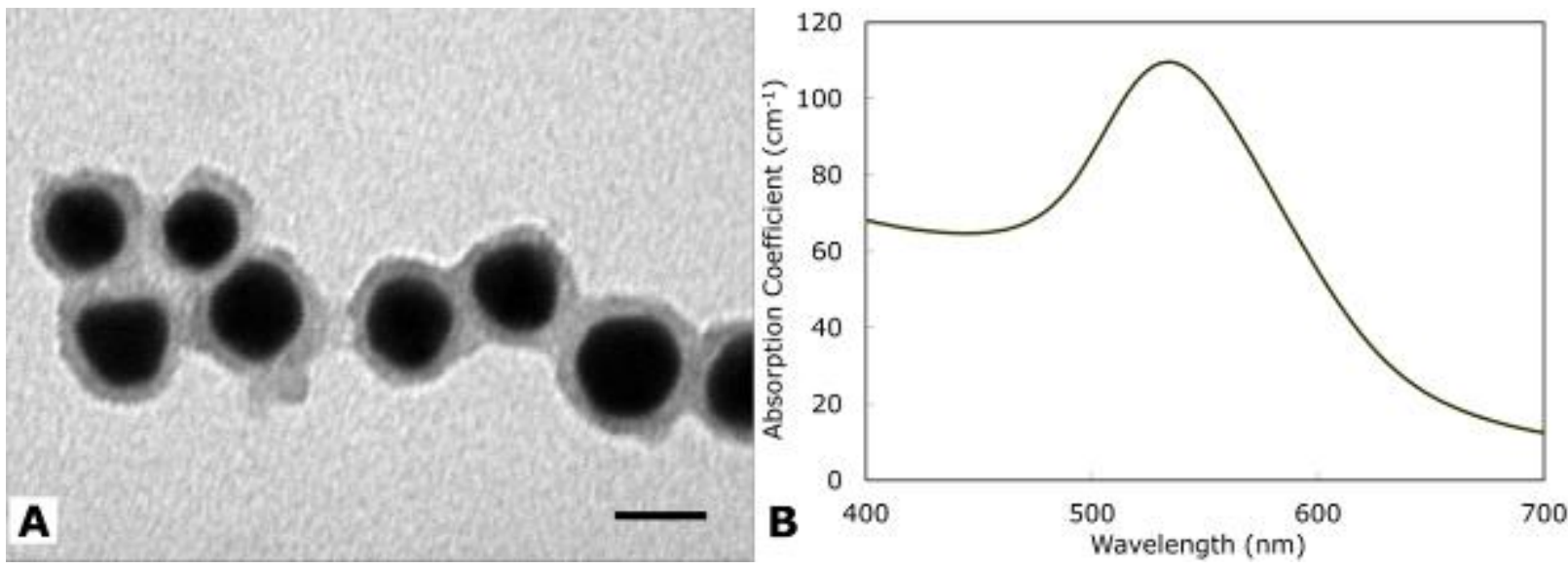

Figure 3.3: Transmission electron microscopy image (A) and optical absorption coefficient (B) of the silica coated gold NP PFH solution. The gold NPs were approximately $20 \mathrm{~nm}$ in diameter with a $5 \mathrm{~nm}$ thick silica coating. The peak optical absorption was between $530-540 \mathrm{~nm}$. The scale bar is $20 \mathrm{~nm}$.

\subsubsection{Sound dispersion effects}

Other studies have demonstrated excellent agreement between the photoacoustic model (equation 3.1) and experiments of optical absorbing mm-sized spherical droplets at photoacoustic frequencies less than $20 \mathrm{MHz}$, where sound dispersion has been neglected [5456]. While PFC liquids have negligible dispersion at $22 \mathrm{MHz}$ [203], a small but measureable increase of sound dispersion was found for three PFC liquids up to $1000 \mathrm{MHz}$ [169]. For $\mathrm{PFH}$, the liquid used in this study, the sound speed increased from $480 \mathrm{~m} / \mathrm{s}$ to $486 \mathrm{~m} / \mathrm{s}$ and the ultrasound attenuation increased from $1368 \mathrm{~dB} / \mathrm{cm}$ to $16840 \mathrm{~dB} / \mathrm{cm}$ from 200 to 1000 $\mathrm{MHz}$ at $36^{\circ} \mathrm{C}$ [169]. Using a power law fit, the attenuation as a function of frequency was found to be $\alpha(f)=0.352 \mathrm{f}^{1.56}$, as described in reference [169]. Attenuation is linked to changes in sound speed via the Kramers-Kronig relations. To obtain a smooth change in 
sound speed with frequency, the sound speed over the range of 100 to $1000 \mathrm{MHz}$ was calculated from the attenuation power law fit of $0.352 \mathrm{f}^{1.56}$ using the Kramers-Kronig relations (equation 3.5).
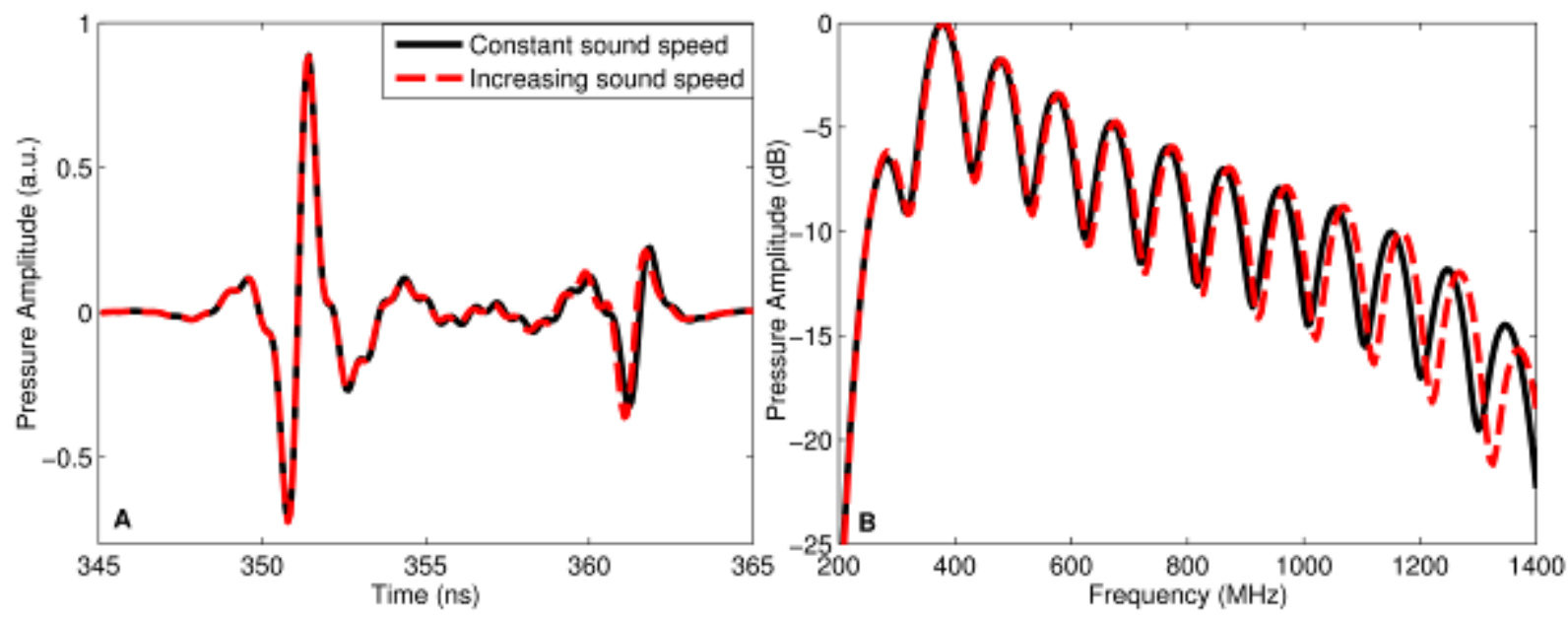

Figure 3.4: The effects of dispersion on the photoacoustic model showing the time domain solution (A) and frequency domain solution (B) for a $5 \mu \mathrm{m}$ droplet using equation 3.1 (constant sound speed, black solid line) and equation 3.2 (sound speed increasing from 480 to $486 \mathrm{~m} / \mathrm{s}$, red dashed line). A Hamming window and 300-1400 MHz bandpass filter were used for both solutions. Good agreement was found up to $300 \mathrm{MHz}$, but discrepancies in the location of the spectral minima and maxima were observed over $500 \mathrm{MHz}$.

\subsubsection{Droplet diameter measurements}

The diameters of 18 micron-sized droplets were calculated using the three signalbased methods (photoacoustic waveform, ultrasound pulse echo and photoacoustic spectrum) and compared to optical microscopy measurements. The results are summarized in table 3.1. 
The diameter was calculated using several methods to verify the photoacoustic spectral fit; in equations 3.1 and 3.2, the spectral minima and maxima shift depending on $q$, which itself depends on the droplet radius and sound speed. The same photoacoustic spectrum could be obtained by varying the droplet radius and sound speed, provided the ratio remained constant. Therefore to ensure the parameters found from the spectral fit were accurate, they were compared to other methods.

The average difference in diameter between the three signal-based methods was 0.12 $\pm 0.09 \mu \mathrm{m}$. The direct optical measurement was an average $0.68 \pm 0.39 \mu \mathrm{m}$ greater than the other three methods. While the three calculated methods based on signal measurement were very close to each other, the optical measurements were $8.5 \%$ higher. The droplet images recorded were only 10-20 pixels across, which significantly reduced the measurement precision. Poor contrast due to difficulties in observing the droplet through the gelatin phantom prevented higher magnification objectives from being used. Due to the number of pixels used and the difficulty in observing the droplet through the phantom, the uncertainty in the droplet diameters estimated from optical methods was determined to be $\pm 0.5 \mu \mathrm{m}$. The larger diameter estimation and uncertainty in the optical method is likely due to the difficulty in determining the edges of a small spherical object near the limits of optical resolution, compounded by the difficulty in seeing through the partially translucent gelatin phantom. The agreement between optical and signal processing methods improved as the droplet diameter increased; for example, the optical method was $13 \pm 8 \%$ greater for diameters $5-7$ $\mu \mathrm{m}$, and was only $4.8 \pm 3.2 \%$ for diameters over $10 \mu \mathrm{m}$. Therefore, it can be inferred that the 
diameter determined through optical microscopy would likely improve if larger diameters or higher magnifications were used. Since it was difficult to determine the droplet diameter visually, using the signal-based measurements were essential to corroborate the diameter as predicted by the model (equation 3.2).

\begin{tabular}{ccccc}
\hline \hline $\begin{array}{c}\text { Droplet } \\
\text { Number }\end{array}$ & Photoacoustic & Droplet Diameter $(\mu \mathrm{m})$ \\
\hline 1 & $5.14 \pm 0.09$ & $5.21 \pm 0.05$ & $5.0 \pm 0.1$ & $6.3 \pm 0.5$ \\
2 & $5.38 \pm 0.09$ & $5.40 \pm 0.05$ & $5.3 \pm 0.1$ & $5.7 \pm 0.5$ \\
3 & $5.42 \pm 0.09$ & $5.62 \pm 0.05$ & $5.4 \pm 0.1$ & $6.9 \pm 0.5$ \\
4 & $5.04 \pm 0.09$ & $5.16 \pm 0.05$ & $5.4 \pm 0.1$ & $5.7 \pm 0.5$ \\
5 & $6.00 \pm 0.09$ & $5.66 \pm 0.05$ & $6.0 \pm 0.1$ & $6.9 \pm 0.5$ \\
6 & $6.14 \pm 0.09$ & $6.22 \pm 0.05$ & $6.1 \pm 0.1$ & $6.3 \pm 0.5$ \\
7 & $6.43 \pm 0.09$ & $6.46 \pm 0.05$ & $6.4 \pm 0.1$ & $7.4 \pm 0.5$ \\
8 & $6.86 \pm 0.09$ & $6.98 \pm 0.05$ & $6.9 \pm 0.1$ & $7.4 \pm 0.5$ \\
9 & $7.10 \pm 0.09$ & $7.20 \pm 0.05$ & $7.0 \pm 0.1$ & $7.4 \pm 0.5$ \\
10 & $7.10 \pm 0.09$ & $7.06 \pm 0.05$ & $7.2 \pm 0.1$ & $8.0 \pm 0.5$ \\
11 & $7.44 \pm 0.09$ & $7.51 \pm 0.05$ & $7.3 \pm 0.1$ & $8.0 \pm 0.5$ \\
12 & $8.11 \pm 0.09$ & $8.21 \pm 0.05$ & $8.1 \pm 0.1$ & $9.1 \pm 0.5$ \\
13 & $7.97 \pm 0.09$ & $8.06 \pm 0.05$ & $8.1 \pm 0.1$ & $8.6 \pm 0.5$ \\
14 & $10.3 \pm 0.1$ & $10.56 \pm 0.06$ & $10.3 \pm 0.1$ & $10.9 \pm 0.5$ \\
15 & $10.7 \pm 0.1$ & $10.82 \pm 0.06$ & $10.7 \pm 0.1$ & $10.9 \pm 0.5$ \\
16 & $13.1 \pm 0.1$ & $13.25 \pm 0.07$ & $13.1 \pm 0.1$ & $14.3 \pm 0.5$ \\
17 & $13.2 \pm 0.1$ & $13.37 \pm 0.07$ & $13.3 \pm 0.1$ & $14.3 \pm 0.5$ \\
18 & $13.4 \pm 0.1$ & $13.56 \pm 0.07$ & $13.3 \pm 0.1$ & $13.7 \pm 0.5$ \\
\hline \hline
\end{tabular}

Table 3-1: The diameters of 18 droplets calculated using the photoacoustic time domain signal (equation 3.3), the ultrasound time domain signal (equation 4), spectral comparison between model and experiment (equation 3.2) and optical microscopy. The uncertainties in the photoacoustic and ultrasound methods were calculated based on the accuracy of determining the locations of the signal peaks in figure 1, and the model uncertainty from the diameter variations in fitting the experiment to theory. The optical method uncertainty was large due to the difficulty in determining the droplet edges, and the limited number of pixels used when acquiring the droplet image. 


\subsubsection{Photoacoustic spectral measurements}

The model (equation 3.2) calculates the photoacoustic pressure wave emitted from a spherical homogeneous liquid undergoing thermoelastic expansion due to absorption of optical energy. To determine if the measured photoacoustic spectra from NP-loaded PFH droplets were in agreement with the model, the photoacoustic signals for three droplets of different sizes were measured; a $5.7 \mu \mathrm{m}$ droplet measured at $375 \mathrm{MHz}$, a $2.45 \mu \mathrm{m}$ droplet measured at $750 \mathrm{MHz}$, and a $6.95 \mu \mathrm{m}$ droplet measured at $750 \mathrm{MHz}$ (figure 3.5). No photoacoustic signal was detected for unloaded PFH droplets. All signals were normalized to maximum amplitude, and the droplet diameter was determined by matching the measured photoacoustic spectral maxima and minima to the model (equation 3.2). The diameter found from the spectral fit agreed well with the time domain photoacoustic and ultrasound methods used to calculate the diameter (see table 3.1).

The measured spectra agreed well with the model in both the time and frequency domains for all three droplets (figure 3.5). The gold NPs act exclusively as the optical absorbing material within the droplets, yet the photoacoustic pressure wave emitted is based entirely on the PFH liquid properties, and not of the gold NPs. This is consistent with previous photoacoustic measurements using lead sulphide NPs inside PFC droplets which showed good agreement in the spectral features between the model and experiment [204, 205]. These results also agree with a study that found the photoacoustic wave is generated from the liquid surrounding the NP, and not from the NP itself [190]. This suggests that the NP-loaded droplets can be modeled as a single photoacoustic source taking into account only 
the liquid PFC properties, irrespective of the optical absorbing NPs contained within, provided they absorb light sufficiently at the wavelength used. As NP-loaded emulsions gain popularity as photoacoustic contrast agents, the photoacoustic signal from droplets can be maximized by optimizing the size, shape and type of NPs knowing that the emulsions will emit pressure waves as a single photoacoustic source. 

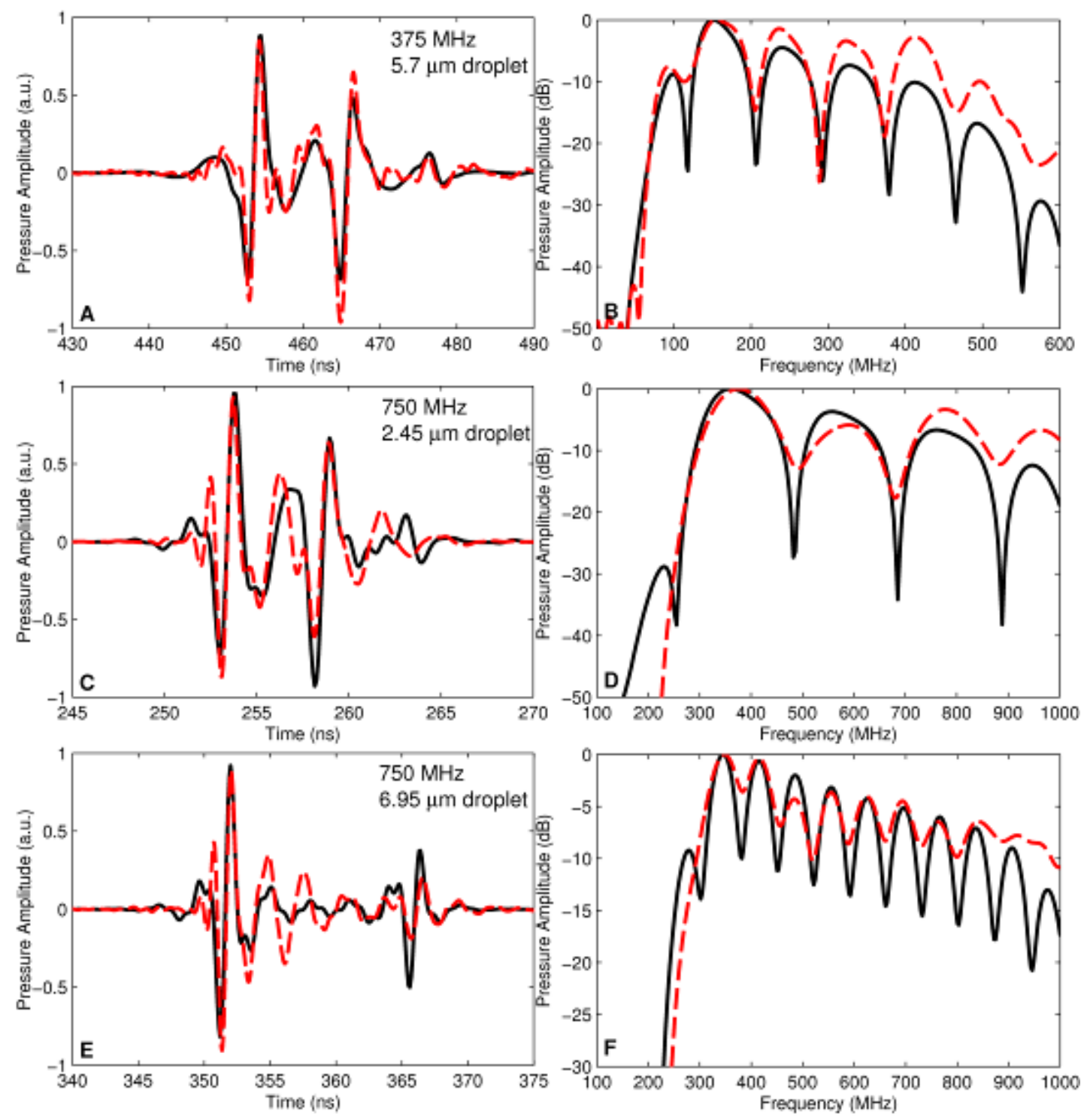

Figure 3.5: The measured photoacoustic signal (red dashed line) compared to the model (black solid line, equation 3.2) for a $5.7 \mu \mathrm{m}$ droplet measured at $375 \mathrm{MHz}(\mathrm{A}, \mathrm{B})$, a $2.45 \mu \mathrm{m}$ droplet measured at $750 \mathrm{MHz}(\mathrm{C}, \mathrm{D})$ and a $6.95 \mu \mathrm{m}$ droplet measured at $750 \mathrm{MHz}(\mathrm{E}, \mathrm{F})$. A bandpass filter of 100-500 MHz was used for the $375 \mathrm{MHz}$ measurement, and a bandpass filter of 300-1000 MHz was used for the $750 \mathrm{MHz}$ measurements. A Hamming window was applied to all measurements. Good agreement between model and experiment were found for the waveforms (A, C, E) and in the location of the spectral minima and maxima $(\mathrm{B}, \mathrm{D}, \mathrm{F})$. 


\subsection{Conclusions}

The photoacoustic spectra of micron-sized NP-loaded PFH droplets measured from $100-1000 \mathrm{MHz}$ were in good agreement to a theoretical model that calculates the pressure wave emitted from an optical absorbing droplet. Due to the fact that good agreement between experiment and theory was achieved using only the PFH liquid physical properties in the model, we conclude that the droplets behave as a single photoacoustic source based on the liquid properties such as the sound speed, density and diameter, and without regard to the NP composition. The NPs and liquids used in emulsions can be chosen to maximize optical absorption, knowing that the size and composition of the optical absorbing NPs have a negligible effect on the photoacoustic spectrum. In addition, four independent methods were used to determine the droplet diameter and corroborate the diameter determined from the photoacoustic spectral calculation. The three methods based on the photoacoustic and ultrasound signal processing methods agreed to within an average of $0.12 \pm 0.09 \mu \mathrm{m}$, while the direct optical microscopy measurement was $0.68 \pm 0.39 \mu \mathrm{m}$ higher. The large errors associated with the optical measurement may be due to limited visibility, poor contrast and the difficulty in delineating the borders of micron-sized objects when embedded in a translucent gelatin medium.

\subsection{Acknowledgements}

E. Strohm is supported through a NSERC doctoral scholarship. This research was undertaken, in part, thanks to funding from NSERC and the Canada Research Chairs 
Program awarded to M. Kolios. Funding to purchase the equipment was provided by the Canada Foundation for Innovation, the Ontario Ministry of Research and Innovation, and Ryerson University. This study was supported, in part, by the Ontario Institute for Cancer Research Network through funding provided by the Province of Ontario, the FY07 Department of Defense Breast Cancer Research Program Concept Award (BC075873), a program project grant entitled "Imaging for Cancer" and "Ultrasound for Cancer Therapy" from the Terry Fox Foundation, and the Ontario Research Fund-Research for Excellence Program. 


\section{Chapter 4}

\section{Probing red blood cell morphology using high frequency photoacoustics ${ }^{2}$}

\subsection{Abstract}

A method that can rapidly quantify variations in the morphology of single red blood cells (RBCs) using light and sound is presented. When irradiated with a laser pulse, a RBC absorbs the optical energy and emits an ultrasonic pressure wave called a photoacoustic wave. The power spectrum of the resulting photoacoustic wave contains distinctive features that can be used to identify the RBC size and morphology. When probing particles 5-10 $\mu \mathrm{m}$ in diameter (such as RBCs) with high frequency photoacoustics unique periodically varying minima and maxima occur throughout the photoacoustic signal power spectrum above frequencies of $100 \mathrm{MHz}$. The location and distance between spectral minima scale with the size and morphology of the RBC; these shifts can be used to quantify small changes in the

\footnotetext{
${ }^{2}$ This chapter is based on an original article published as: E.M. Strohm, E. Berndl, and M. C. Kolios. "Probing Red Blood Cell Morphology Using High Frequency Photoacoustics.” Biophysical Journal 105(1), 2013, pp. 5967.
} 
morphology of RBCs. Morphological variations from their normal bi-concave shape are commonly associated with disease or infection. Using a single wide-bandwidth transducer sensitive to frequencies between 100 and $500 \mathrm{MHz}$, we were able to differentiate healthy RBCs from irregularly shaped RBCs (such as echinocytes, spherocytes and swollen RBCs) with high confidence using a sample size of just 21 RBCs. As each measurement takes only seconds, these methods could eventually be translated to an automated device for rapid characterization of RBC morphology, and deployed in a clinical setting to help diagnose RBC pathology.

\subsection{Introduction}

Red blood cells (RBCs) have a flexible biconcave disk-shape that enables efficient transport of oxygen to the peripheral cells of the body through the narrow and tortuously winding capillary system [206]. Disease, infection, genetic disorders and variations in blood chemistry can alter the RBC shape, reducing their ability to bend and deform [207]. Abnormal RBC morphologies can impede or even obstruct the circulation, causing tissue necrosis in severe cases.

Clinical diagnoses of blood disorders use a set of indices to provide information about individual $\mathrm{RBC}$ physical characteristics. The average $\mathrm{RBC}$ is approximately $7.8 \mu \mathrm{m}$ in diameter, 1-2 $\mu \mathrm{m}$ in height and has a $94 \mu \mathrm{m}^{3}$ volume [208]. Current automated diagnostic methods use electrical impedance or light scattering methods to determine the RBC concentration and their mean corpuscular volume $[209,210]$. The diameter is inferred from 
the volume as they are directly related for healthy RBCs [211], however this relation may break down for abnormally shaped RBCs. These tools cannot determine the dimensions or shape directly, and only indicate if a sample is outside of accepted guidelines. Their limited capability to diagnose the pathology requires additional testing to identify abnormal RBC morphologies such as spherocytes (spherical RBCs) or echinocytes (crenated shape with protrusions). Methods to determine the individual RBC shape through blood smears or optical interference methods [212] are laborious and time intensive, prohibiting analysis of large samples sizes.

RBCs contain large amounts of hemoglobin, a molecule capable of binding oxygen. Hemoglobin also significantly absorbs visible light, while other tissues do not, thus making blood an ideal contrast agent for photoacoustic imaging in vivo [213]. After absorbing energy, the particles rapidly increase in temperature and pressure, resulting in a thermoelastic expansion and the emission of a photoacoustic wave. These waves can be detected using conventional ultrasound transducers with frequency sensitivities typically between 10-40 $\mathrm{MHz}$ [64]. This effect has been used in-vivo for functional imaging [139], photoacoustic tomography [65], the detection of tumors [138] and imaging vasculature with mm-scale resolution $[114,140]$. The photoacoustic effect can be exploited on the micro-scale using various photoacoustic microscopes $[67,68]$. In this system, conventional ultrasound transducers are combined with a highly focused laser to create in-vitro and in-vivo micrometer-resolution images of vasculature [60] and even single cells [214]. Current photoacoustic microscopes use inexpensive conventional transducers to produce stunning 
micro-sized images with excellent contrast; however they have a limited photoacoustic frequency range of operation.

Our photoacoustic microscope uses transducers with frequencies over $100 \mathrm{MHz}$ with bandwidths in the hundreds of $\mathrm{MHz}$ [215]. Like other photoacoustic microscopes, it can image with micrometer resolution $[128,144]$. However the distinct advantage of this microscope is the broad ultrasound/photoacoustic frequency bandwidth which can be used for a quantitative analysis of micron-sized particles [175]. For any particle in the 1-50 $\mu \mathrm{m}$ size range (such as RBCs), unique features in the photoacoustic spectrum over $100 \mathrm{MHz}$ are observed. These periodically varying spectral minima and maxima depend strongly on the size, morphology, orientation and composition of the particle [55]. Quantitative analysis of the photoacoustic spectra can be used to help identify these parameters and extract information from the particle examined.

The size, shape and intrinsic optical absorption properties of RBCs make them ideal candidates for high frequency quantitative photoacoustic methods. The photoacoustic signal can be used to infer the RBC size and shape, and thus give further insight into the specific abnormality observed than current clinical methods. These methods could be translated into an automated device capable of measuring a large sample size for a rapid determination of RBC morphology and thus pathology.

\subsection{Materials and Method}

RBCs were extracted from a healthy male volunteer in accordance with the Ryerson 
Ethics Board (REB \#2012-210). A drop of blood was drawn from the finger using a lancet and immediately deposited in $1 \mathrm{~mL}$ Dulbecco's Modified Eagle Medium (DMEM) containing $10 \%$ fetal bovine serum to maintain viability (Sigma Aldrich, USA). All measurements were made on $35 \mathrm{~mm}$ glass bottom dishes (Mattek Corporation, USA). The dishes were coated with a thin layer of $0.5 \%$ agar approximately $200 \mu \mathrm{m}$ thick to reduce photoacoustic back reflections from the glass substrate. Approximately $30 \mu \mathrm{L}$ of the DMEMRBC solution was deposited into $2 \mathrm{~mL}$ of coupling fluid inside the glass bottom dish to dilute the sample.

For the orientation and echinocyte measurements, DMEM was used as the coupling fluid. For the osmolality measurements, phosphate buffered saline (PBS) was used as the coupling fluid. The osmolality of the PBS and DMEM liquids were measured three times each using a Vapro 5520 vapor pressure osmometer (Wescor, USA) and were found to be an average of 294 and $332 \mathrm{mmol} / \mathrm{kg}$, respectively. The standard deviation of three measurements was $\pm 10 \mathrm{mmol} / \mathrm{kg}$. The osmolality of the PBS solutions were adjusted by adding water. For the ATP depletion procedure, the extracted blood was stored in PBS. A small sample of the PBS-RBC solution was deposited on the glass bottom dish containing 2 $\mathrm{mL}$ DMEM and $21 \mathrm{RBCs}$ were measured. The PBS-RBC solution was stored at $4^{\circ} \mathrm{C}$ for 24 hours to induce echinocyte formation, and then a small sample was added to a glass bottom dish containing $2 \mathrm{~mL}$ PBS. $21 \mathrm{RBCs}$ were then measured. Another PBS-RBC sample was then added to a glass bottom dish containing $2 \mathrm{~mL}$ DMEM and then $21 \mathrm{RBCs}$ were measured. All measurements were performed at $36^{\circ} \mathrm{C}$. 
All measurements were completed using a photoacoustic microscope developed by Kibero GmbH. It is an Olympus IX81 inverted optical microscope fitted with a transducer above the sample stage (Figure 4.1A). The sample was positioned between the optical objective and transducer. The system optics allows for viewing the sample and aligning the transducer; it also focuses the laser onto the sample. A dilute suspension of freshly extracted human RBCs was deposited into the liquid in the sample holder. Most RBCs would fall to the substrate and align horizontally, but some would initially come to rest in a vertical orientation (Figure 4.1B); these would eventually tip over to a horizontal orientation. The laser was focused onto single RBCs and the transducer recorded the resulting photoacoustic wave (Figure 4.1C). The photoacoustic signals of RBCs were measured in both a vertical and horizontal orientation. The diameter was obtained from the optical images for comparison to theoretical simulations. A transducer with center frequency $375 \mathrm{MHz}$ and $\mathrm{f}$-number $=1$ (Kibero $\mathrm{GmbH}$, Germany) was used for all measurements. A $532 \mathrm{~nm}$ laser (Teem Photonics, France) was focused by a 10x optical objective to approximately a $10 \mu \mathrm{m}$ spot size onto the sample, sufficient to irradiate a single RBC. The laser had a pulse width of $330 \mathrm{ps}$ and a pulse repetition frequency of $4 \mathrm{kHz}$. Signals were amplified by a $40 \mathrm{~dB}$ amplifier (Miteq, USA), digitized $8 \mathrm{GS} / \mathrm{s}$ (DC252, Acqiris, USA) and were averaged 100-200 times to increase the signal-to-noise ratio. The laser power was measured at the objective using a Nova II power meter and PD10 low energy sensor (Ophir, Israel). The laser power was adjusted so that the laser fluence was $20-150 \mathrm{~mJ} / \mathrm{cm}^{2}$ at the sample. For the orientation measurements, photoacoustic signals were recorded approximately once a second. For all other 
measurements, the RBC was centered over the laser spot and the photoacoustic signal recorded. A Hamming window was applied to the measured signals, then the normalized power spectrum $P(f)$ was calculated using

$$
P(f)=20 \log _{10}\left|\frac{P_{m}(f)}{P_{n}(f)}\right|
$$

where $P_{m}(f)$ is the Fourier Transform of the measured signal $p_{m}(t)$, and $P_{\mathrm{n}}(f)$ is the Fourier transform of $p_{n}(t)$, which is the spectral response of the transducer system and electronics. This normalization signal is used to remove any artifacts due to the transducer/electronics of the system; it is generated by a $200 \mathrm{~nm}$ thick gold film which was spectrally flat over the transducer bandwidth with variations of less than $3 \mathrm{~dB}$ from 100-1000 $\mathrm{MHz}$. The normalization signal is typically recorded using pulse-echo ultrasound from an ultrasonically flat reflective surface [118], however the transducer bandwidth for photoacoustic measurements is larger than pulse echo ultrasound. Pulse-echo ultrasound waves also travel twice the distance as photoacoustic waves that are emitted from the sample, and therefore are attenuated more strongly than photoacoustic waves. More specific details of the equipment, signal processing methods and normalization procedure can be found in $[106,175]$ and in section 1.5. 
a

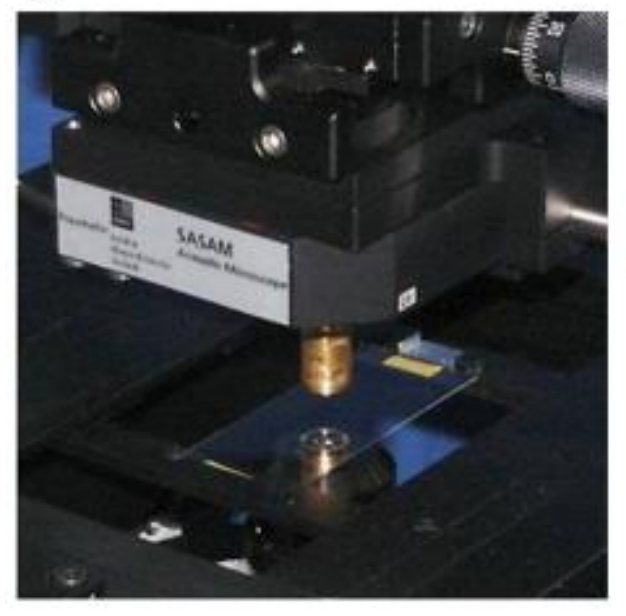

b

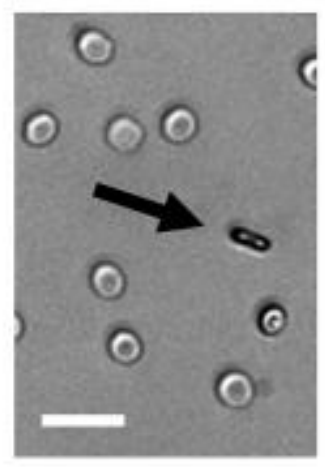

c

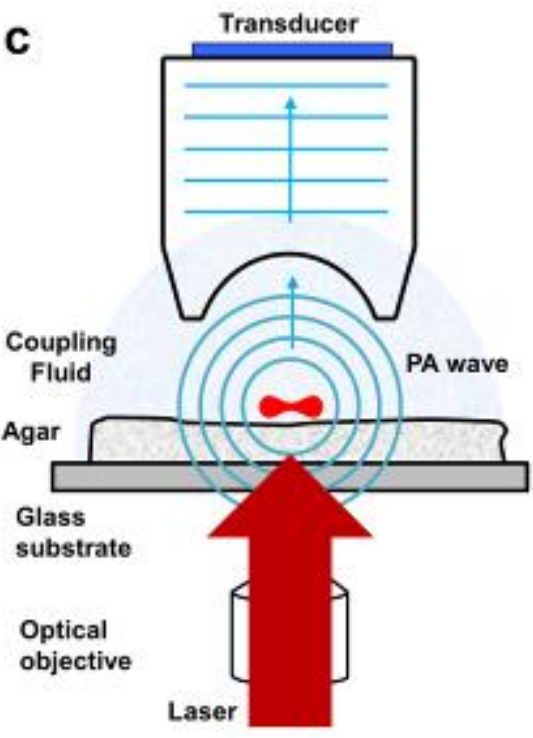

Figure 4.1: The photoacoustic microscope. (a) The SASAM photoacoustic microscope. The sample is positioned between the transducer and optical objective. (b) RBCs after deposition into the sample holder. Most RBCs align horizontally on the substrate, however a small number align vertically as indicated by the arrow. The scale bar is $20 \mu \mathrm{m}$. (c) A schematic showing the sample positioning relative to the transducer and focused laser.

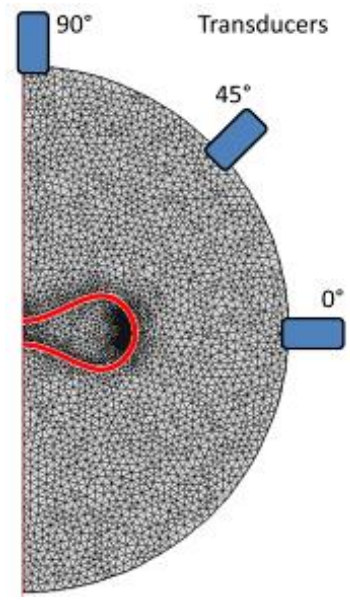

Figure 4.2: The finite element model. The finite element model developed to describe the photoacoustic waves generated by micron sized particles. The RBC was positioned at the center, and transducers recorded the emitted photoacoustic wave around the RBC. 
A finite element model (FEM) was used to validate the measured results and determine how the photoacoustic spectral features vary with different parameters such as the diameter and orientation. The FEM was developed in COMSOL Multiphysics. A biconcave shape using the equation developed by Fung et al [216] was positioned at the center of a 2D axi-symmetric $10 \mu \mathrm{m}$ radius spherical system (Figure 4.2). A mesh size of $0.05 \mu \mathrm{m}$ was used. A density of $1100 \mathrm{~kg} / \mathrm{m}^{3}[217,218]$ and a sound speed of $1650 \mathrm{~m} / \mathrm{s}$, similar to the sound speed measured in $[219,220]$ was used as input parameters. The model was initiated with the $\mathrm{RBC}$ at a pressure higher than the surrounding fluid to simulate the thermoelastic expansion that occurs immediately after absorption of energy. A transient acoustics solver was used to calculate the ultrasonic pressure wave propagation from the $\mathrm{RBC}$ as a function of time. The signal was recorded at the system boundary at various angles around the RBC. Further details on the development of the model can be found in [81].

\subsection{Results}

\subsubsection{Numerical simulations}

Numerical simulations were performed using the FEM to compare our measurements to theoretical predictions. In these simulations, the shape of the photoacoustic spectrum was strongly dependent on the RBC size and orientation. Due to the large aspect ratio of the RBC, the photoacoustic wave generated has distinctly different spectral features depending on the relative angle of observation (Figure 4.3A). When a $7.82 \mu \mathrm{m} \mathrm{RBC}$ is oriented horizontally towards the transducer (denoted $90^{\circ}$ in Figure 4.2), the spectrum is essentially featureless 
from $100-500 \mathrm{MHz}$, with the first spectral minima occurring at $800 \mathrm{MHz}$. As the RBC rotates to a vertical orientation, the spectral amplitude decreases and the spectral minimum shifts from approximately $800 \mathrm{MHz}$ (at $90^{\circ}$, horizontal orientation) to $250 \mathrm{MHz}$ (at $0^{\circ}$, vertical orientation). The RBC diameter also affects the location of the spectral minima (Figure 4.3B). When oriented vertically, the first spectral minimum shifts from $200 \mathrm{MHz}(8.80 \mu \mathrm{m}$ diameter) to $270 \mathrm{MHz}(6.60 \mu \mathrm{m}$ diameter $)$.

\subsubsection{Photoacoustic signals vs. RBC orientation}

Photoacoustic signals were recorded from a single $7.8 \mu \mathrm{m}$ diameter RBC first in the vertical orientation, then in a horizontal orientation after it fell over. The time domain signal as recorded in both orientations is shown in Figure 4.4A. Aside from the change in signal amplitude, it is difficult to infer any details about the RBC from these signals alone. Figure 4.4B shows the power spectrum of the signals from Figure 4.4A for the same RBC. In the vertical orientation, periodically varying minima and maxima are observed throughout the spectrum starting at $200 \mathrm{MHz}$. In the horizontal orientation, the photoacoustic spectrum is nearly flat and featureless over the bandwidth of the transducer (approximately 150-550 MHz). Good agreement between our measurements and FEM simulations was observed for the $7.8 \mu \mathrm{m} \mathrm{RBC}$ oriented vertically and horizontally (Figure 4.4B). To examine how the signal varied with angle, an animation showing the photoacoustic time domain signal, power spectrum and optical images of a single RBC tipping from a vertical to horizontal orientation is provided in the supplementary materials (Movie S1). A gradual increase in the frequency and amplitude of the spectral minima and maxima was observed as the RBC rotated from the 
vertical to horizontal orientation, agreeing with theoretical predictions (Figure 4.3A).

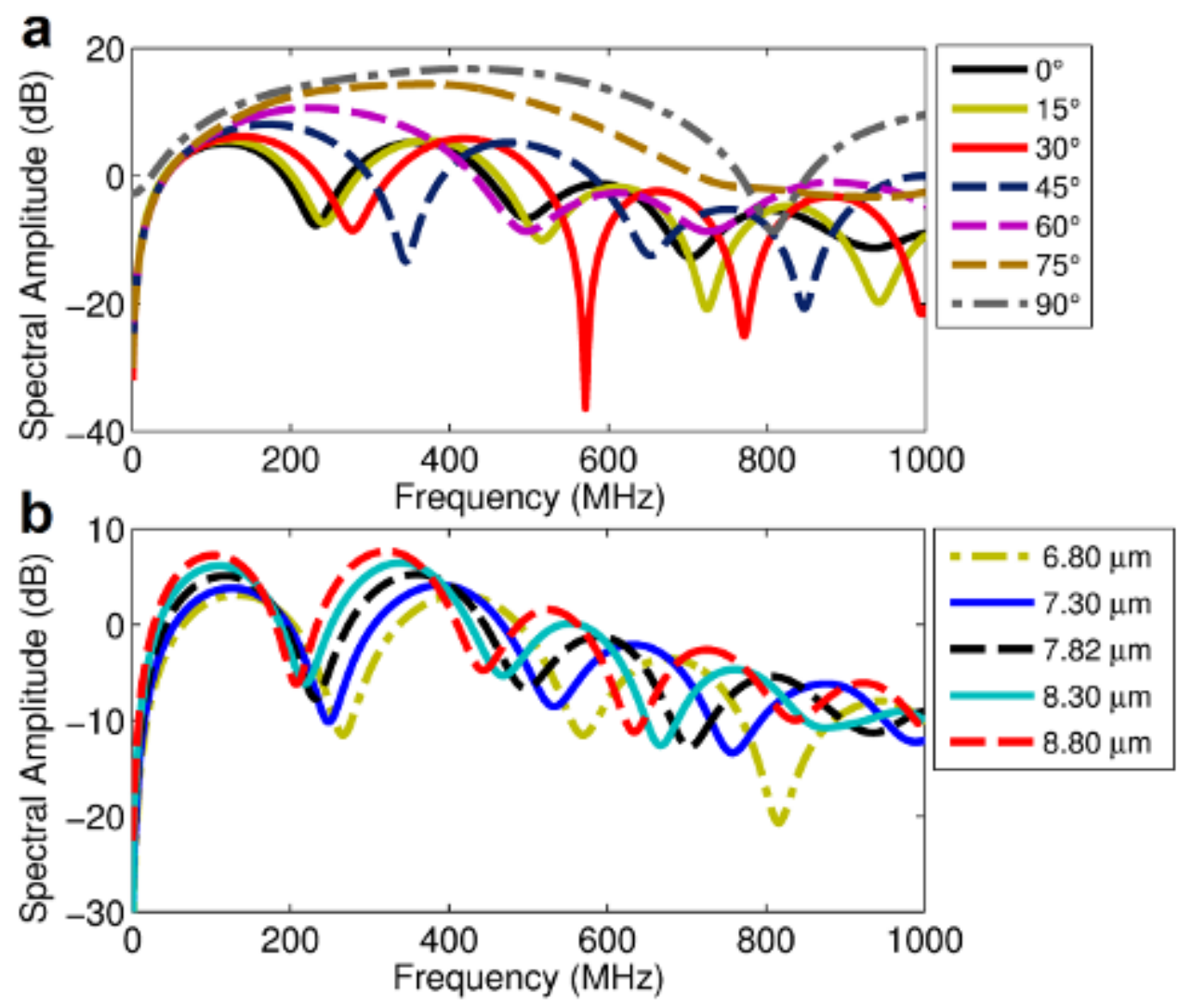

Figure 4.3: Numerical simulations results using the finite element model. (a) The photoacoustic spectrum as the transducer position was varied from $0^{\circ}$ (vertical RBC) to $90^{\circ}$ (horizontal RBC) for a $7.82 \mu \mathrm{m}$ diameter RBC. (b) The photoacoustic spectrum calculated from a vertical RBC as the diameter was varied from $6.80 \mu \mathrm{m}$ to $8.80 \mu \mathrm{m}$. 

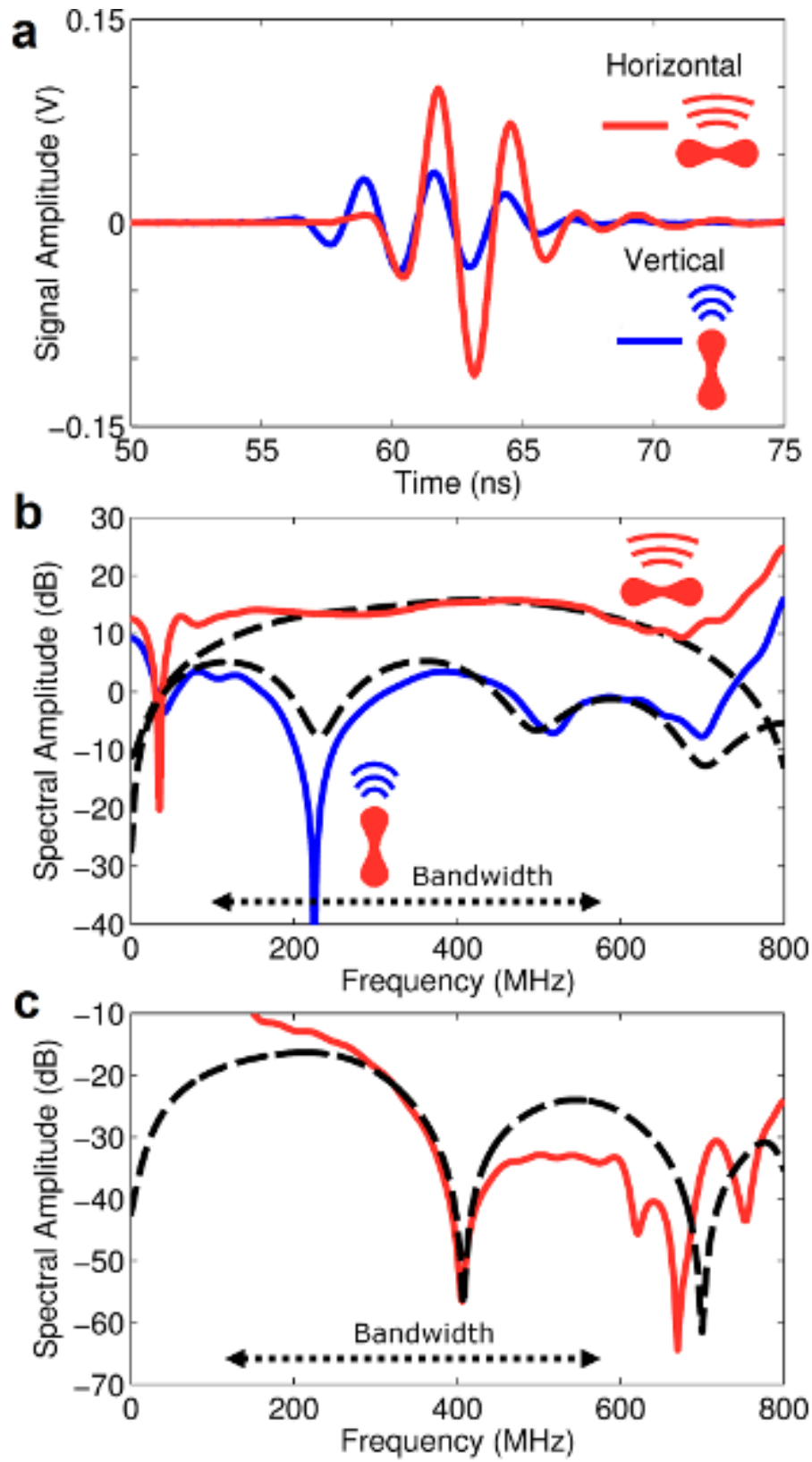

Figure 4.4: Photoacoustic measurements of single RBCs. (a) The time domain signal as measured from a single $7.8 \mu \mathrm{m}$ diameter RBC when aligned vertically (blue) and horizontally (red) relative to the transducer measured in PBS at isotonic conditions. (b) The photoacoustic power spectrum of the RBCs shown in Figure 4.3A when aligned vertically (blue) and horizontally (red) compared to the numerical simulations for their respective orientation (dashed black line). (c) The photoacoustic 
spectrum of a $5.8 \mu \mathrm{m}$ diameter RBC induced into a spherical shape by immersion in a hypotonic solution of $84 \mathrm{mmol} / \mathrm{kg}$ (solid red line) compared to numerical simulations of a spherical morphology (dashed black line). A schematic of the RBC shape and orientation is shown inset within the figure. The transducer bandwidth is from about 150 to $550 \mathrm{MHz}$ as indicated by the dotted arrow. The signal amplitude has been normalized to match theory.

\subsubsection{Photoacoustic signals vs. RBC morphology}

RBCs maintain their biconcave shape in isotonic conditions (approximately 300 $\mathrm{mmol} / \mathrm{kg}$ ). When RBCs are immersed in a hypotonic solution, the RBC swells, with the swelling increasing as the osmolality decreases until they eventually form spherocytes [221]. The photoacoustic signals from spherical RBCs were investigated by immersing healthy RBCs in a hypotonic solution of $84 \mathrm{mmol} / \mathrm{kg}$. The diameter and spherical morphology were confirmed optically. Figure $4.4 \mathrm{C}$ shows the power spectrum of a $5.8 \mu \mathrm{m}$ diameter spherical RBC compared to theoretical predictions using the FEM. Good agreement in the location of the spectral features between measurements and theory was observed, and these results were distinctly different than those observed from the biconcave shape in any orientation (Fig. 4B). The sound speed used also affects the location of the spectral minima. When compared to spherical droplets containing perfluorohexane liquid instead of blood (sound speed 480 $\mathrm{m} / \mathrm{s}$ vs. $1650 \mathrm{~m} / \mathrm{s}$ ), the spectral minima shift to lower frequencies and more spectral minima were observed (see Figure 3.5A, B).

We showed in Figure 4.4 that the photoacoustic power spectrum varied depending on the RBC orientation and morphology. For a RBC aligned horizontally, the spectrum was 
fairly flat over the $150-550 \mathrm{MHz}$ bandwidth of the transducer. In this orientation, the signal depends on the RBC thickness; for a healthy RBC, the thickness is less than $1 \mu \mathrm{m}$ at the center. As a RBC swells, the thickness increases which affects the photoacoustic signal. Figure $4.5 \mathrm{~A}$ shows the average power spectrum of $21 \mathrm{RBCs}$ in a horizontal orientation immersed in PBS ranging from isotonic (294 mmol/ $\mathrm{kg}$ ) to hypotonic conditions (84 $\mathrm{mmol} / \mathrm{kg}$ ), where the error bars indicate the standard deviation of the averaged measurements. As the osmolality decreases, the RBC swells, eventually forming a spherical shape below $120 \mathrm{mmol} / \mathrm{kg}$. At frequencies above $300 \mathrm{MHz}$, the spectral amplitude gradually decreases as the osmolality decreases. Below $147 \mathrm{mmol} / \mathrm{kg}$, distinct spectral minima can be observed between 300 and $400 \mathrm{MHz}$, similar to those observed in Figure 4.4C. These spectral features indicate the RBCs are becoming spherical (at $147 \mathrm{mmol} / \mathrm{kg}$ ) or are spherical (84 and $118 \mathrm{mmol} / \mathrm{kg}$ ) which was confirmed through optical imaging. Most of the RBCs at each osmolality exhibited similar spectra. All 21 spectra for the $294 \mathrm{mmol} / \mathrm{kg}$ (Figure 4.5B) and $147 \mathrm{mmol} / \mathrm{kg}$ (Figure 4.5C) are shown to demonstrate the consistency between measurements.

When the adenosine triphosphate (ATP) energy stores of a RBC are depleted, the RBCs form irregularly shaped echinocytes that have spiky protrusions called spicules over the surface $[222,223]$. This process is sometimes reversible depending on how they were formed. The photoacoustic signals from echinocytes were compared to normal RBCs. The photoacoustic power spectrum of freshly extracted human RBCs was measured in cell culture medium to determine the baseline spectrum. The RBCs were then immersed in PBS for 24 
hours to deplete the ATP to form echinocytes, and then the photoacoustic power spectrum measured. Immediately following, the RBCs were added to cell culture medium to replenish the depleted ATP and restore the natural RBC shape. After 15 minutes, the photoacoustic spectrum was measured. A total of 21 RBCs were measured at each stage. Figure 4.6A shows the power spectrum of RBCs in these three states along with representative images. Visually, the echinocytes appeared as an irregularly shaped spiculated cell, differing from the biconcave shape or induced spheres observed previously. The average echinocyte spectrum appears to have a similar spectral shape to swelled RBCs in a hypotonic solution (147 $\mathrm{mmol} / \mathrm{kg}$ Figure $4.5 \mathrm{C}$ ), where both have a minimum around $400 \mathrm{MHz}$. However several features differentiate the swelled RBCs from echinocytes. The decrease in amplitude from the spectral maximum (around $200-250 \mathrm{MHz}$ ) to the spectral minimum (400 MHz) was about half of what was observed, $15 \mathrm{~dB}$ for the echinocytes compared to $30 \mathrm{~dB}$ for the swelled RBCs. The echinocyte measurements also had a higher standard deviation, which can be explained by the variations in spectral shape of each individual RBC (Figure 4.6B). Furthermore, the second spectral peak that occurs around $500-600 \mathrm{MHz}$ for the swelled RBCs is not present for the echinocytes. The spectral variations are likely due to the irregular shape of the echinocytes, and can be used to help differentiate the echinocyte from other morphologies. After immersion in the cell culture medium for 15 minutes, the RBCs rebounded back to their normal biconcave shape. The spectrum was similar to what was observed prior to ATP depletion with similar standard deviations as shown in Figure 4.6, with minimal variation between the 21 measurements (Figure 4.6C). Optical imaging 
confirmed that the RBCs had returned to their normal shape (shown inset in Figure 4.6A).
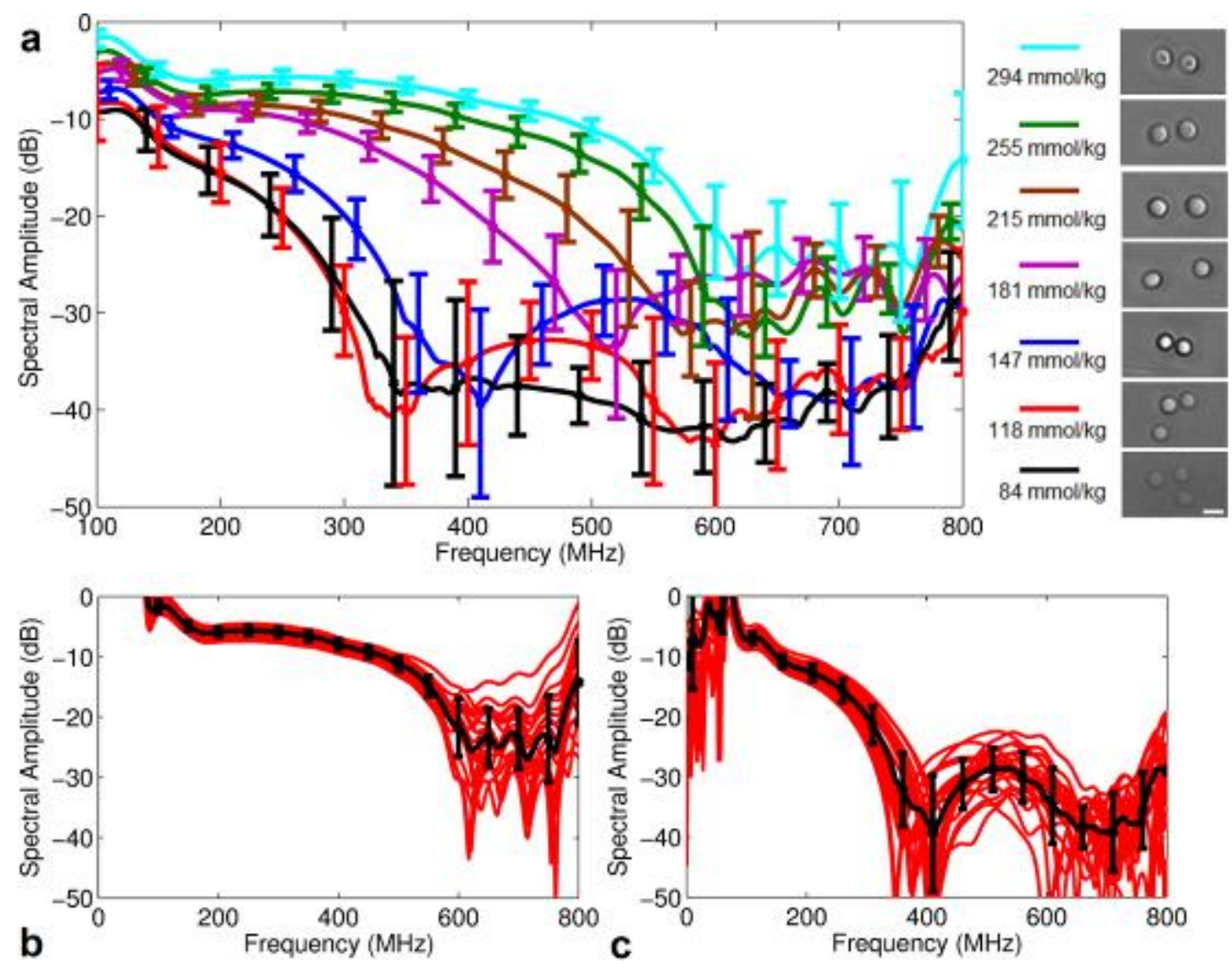

Figure 4.5: Photoacoustic spectral variations with RBC morphology. (a) The photoacoustic spectrum from an average of $21 \mathrm{RBCs}$ measured in PBS with decreasing osmolality. The error bars indicate the standard deviation. As the osmolality decreases, the RBCs swell resulting in a gradual decrease of the photoacoustic spectral amplitude. Optical images confirm the change in morphology, however it is difficult to quantify the change. The scale bar is $10 \mu \mathrm{m}$. (b, c) The photoacoustic spectrum of each individual RBC measurement (red), and the average (black) of all 21 measurements at $294 \mathrm{mmol} / \mathrm{kg}$ (b) and $147 \mathrm{mmol} / \mathrm{kg}(\mathrm{c})$. 

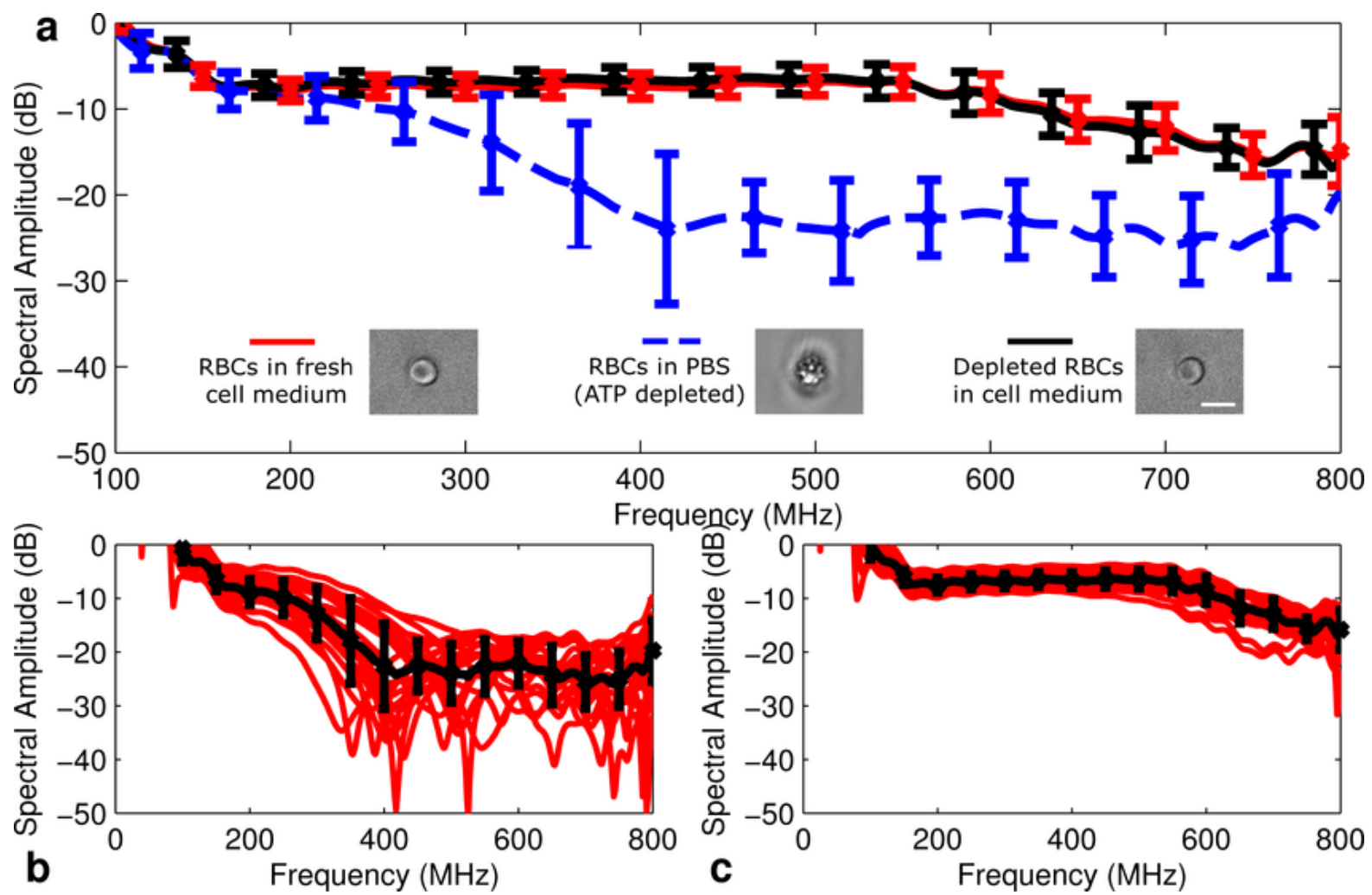

Figure 4.6: Photoacoustic spectrum of healthy RBCs and echinocytes. (a) The photoacoustic spectrum of RBCs in DMEM cell culture medium (red), left in PBS for 24 hours resulting in the formation of echinocytes (black), then the echinocytes deposited in cell culture medium to restore the normal biconcave shape (blue). Images of the RBCs at the three stages are shown inset. The photoacoustic spectrum of the echinocytes is very different than the normal RBCs. The spectrum between the RBCs in fresh medium (red) and ATP-depleted RBCs in medium (blue) are similar, showing that the echinocytes can be restored to their normal biconcave shape. The scale bar is $10 \mu \mathrm{m}$. (b, c) The photoacoustic spectrum of each individual RBC measurement (red) and the average (black) of all 21 measurements of echinocytes in PBS (b) and then in medium (c). A much larger variation between spectra is observed for the echinocytes compared to normal or swollen RBCs. 


\subsubsection{Quantitative Spectral Analysis}

In our measurements, the spectral amplitude $(\mathrm{dB})$ and spectral slope $(\mathrm{dB} / \mathrm{MHz}$, change in signal vs. frequency) within a narrow bandwidth of the spectrum were used to quantify changes in the photoacoustic signals. The slope between $275-300 \mathrm{MHz}$ and the amplitude at $288 \mathrm{MHz}$ for the spectra in Figure 4.5 (spectrum vs. osmolality) and 6 (spectrum vs. ATP depletion) are presented in tables 1 and 2 respectively, where the error is given by the standard deviation between the 21 measurements. This portion of the spectrum was chosen as it occurs before the first significant minimum at $320 \mathrm{MHz}$, and shows variations not observed at lower frequencies. A one-sided t-test was used to test for statistical significance between samples. This test uses the mean, standard deviation and number of samples to determine if two measurements are similar or statistically significant. A statistically significant change in the spectral slope and amplitude was observed between each change in osmolality from 294 to $118 \mathrm{mmol} / \mathrm{kg}$ (p < 0.001). From 118 to $84 \mathrm{mmol} / \mathrm{kg}$, the change in spectral amplitude and spectral slope was not significant $(\mathrm{p}>0.05)$. This was likely because the RBCs were spherical or nearly spherical at these two osmolalities [208], and reducing the osmolality further had no effect. For the echinocyte data presented in Figure 4.6, a significant difference $(\mathrm{p}<0.001)$ in the spectral amplitude and slope was observed between RBCs that have been ATP depleted in PBS compared to either freshly extracted RBCs in medium (prior to ATP depletion) or echinocytes after immersion in medium (to restore the ATP). Comparing the differences between the freshly extracted RBCs in medium and echinocytes in medium (ATP was restored and the echinocytes regained their normal 
biconcave shape), the spectral amplitude was not statistically significant $(\mathrm{p}>0.05)$ however there was a small but statistically significant difference in the spectral slope $(\mathrm{p}<0.01)$.

\begin{tabular}{ccc}
\hline $\begin{array}{c}\text { Osmolality } \\
(\mathbf{m m o l} / \mathbf{k g})\end{array}$ & $\begin{array}{c}\mathbf{2 8 8} \mathbf{~ M H z} \\
\text { Spectral amplitude } \\
(\mathbf{d B})\end{array}$ & $\begin{array}{c}\mathbf{2 7 5 - 3 0 0 ~ M H z} \\
\text { Spectral slope } \\
(\mathbf{d B} / \mathbf{M H z})\end{array}$ \\
\hline 294 & $-5.8 \pm 0.7$ & $-0.006 \pm 0.004$ \\
255 & $-7.4 \pm 0.9$ & $-0.009 \pm 0.004$ \\
215 & $-9.3 \pm 1.1$ & $-0.022 \pm 0.006$ \\
181 & $-11.0 \pm 1.1$ & $-0.042 \pm 0.012$ \\
147 & $-18.1 \pm 2.3$ & $-0.112 \pm 0.026$ \\
118 & $-26.1 \pm 3.7$ & $-0.217 \pm 0.073$ \\
84 & $-25.2 \pm 5.4$ & $-0.176 \pm 0.084$ \\
\hline
\end{tabular}

Table 4-1: Quantitative parameters for RBCs in PBS with varying osmolalities. The spectral amplitude and spectral slope were calculated between 275-300 MHz in Fig. 5 for the RBCs immersed in PBS with osmolalities ranging from 294 to $84 \mathrm{mmol} / \mathrm{kg}$. A statistically significant difference in the spectral amplitude and spectral slope was found between each change in osmolality from 294 to 118 $\mathrm{mmol} / \mathrm{kg}(\mathrm{p}<0.001)$ but not between 118 and $84 \mathrm{mmol} / \mathrm{kg}$. At these two osmolalities, the RBCs were spherical and the spectral shapes are similar.

\begin{tabular}{ccc}
\hline Red blood cell state & $\begin{array}{c}\mathbf{2 8 8} \mathbf{~ M H z} \\
\text { Spectral amplitude } \\
\mathbf{( d B )}\end{array}$ & $\begin{array}{c}\mathbf{2 7 5 - 3 0 0 ~ M H z} \\
\text { Spectral slope } \\
\text { (dB/MHz) }\end{array}$ \\
\hline $\begin{array}{c}\text { Fresh RBCs in culture medium } \\
\text { (Normal biconcave shape) }\end{array}$ & $-7.4 \pm 1.3$ & $-0.011 \pm 0.004$ \\
ATP-depleted RBCs in PBS \\
$\quad$ (Echinocytes) \\
$\begin{array}{c}\text { ATP-depleted RBCs in culture medium } \\
\text { (Restored biconcave shape) }\end{array}$ & $-11.7 \pm 4.1$ & $-0.078 \pm 0.045$ \\
\hline
\end{tabular}

Table 4-2: Quantitative parameters of healthy and abnormal RBCs. The spectral amplitude and spectral slope were calculated between 275-300 MHz in Fig. 6 for fresh RBCs in DMEM, echinocytes in PBS, and then the same echinocytes deposited in DMEM. The echinocytes were 
formed by immersing the fresh RBCs in PBS for 24 hours, which then regained their normal biconcave shape after immersion in DMEM. A statistically significant difference in the spectral amplitude and slope was observed between fresh RBCs and the echinocytes $(\mathrm{p}<0.001)$. No statistically significant difference was observed between fresh RBCs and the depleted RBCs restored in DMEM $(p<0.05)$, however there was a small difference in the spectral slope $(p<0.01)$.

\subsection{Discussion}

Blood cells have been studied extensively using ultrasound [224-226], however there have been limited studies using the closely related photoacoustics method. These experiments demonstrate the first quantitative analysis of any type using photoacoustic frequencies above $100 \mathrm{MHz}$. The photoacoustic microscope is capable of high-resolution imaging, similar to that of other photoacoustic microscopes that use lower frequency transducers; however unlike other photoacoustic microscopes, it can also be used for a broadband signal analysis using frequency bandwidths in the hundreds of MHz. Below 100 $\mathrm{MHz}$, the photoacoustic power spectrum of cells is generally featureless and the shape or orientation of RBCs does not significantly affect the spectral shape or amplitude. As demonstrated in Figure 4.3 and Figure 4.4, distinct minima and maxima occur only above $100 \mathrm{MHz}$, where shifts of hundreds of $\mathrm{MHz}$ with amplitude variations of over $10 \mathrm{~dB}$ occur as the $\mathrm{RBC}$ rotates. This is due to the large aspect ratio of the $\mathrm{RBC}$ (see section 4.4.1 for details). For RBCs measured vertically, our simulations have shown that the spectral minima and maxima shift to lower frequencies as the RBC diameter increases. The diameter can be determined from the spectral features if the orientation of the particle is known. Our goal is 
to translate this new measurement method to an automated flow system capable of measuring thousands of cells per second; however there are several obstacles that must be overcome. For the sequential scanning of the SASAM photoacoustic microscope, the RBCs naturally align horizontally on the substrate, and the RBC can be viewed during the measurements for confirmation of its orientation. When translated to a flow system, RBCs could be made to flow in a horizontal or random orientation using standing waves [227] or specific flow conditions [228, 229]. The analysis methods could be improved by including a second ultrasound transducer positioned at a specific angle from the first transducer. This would record the photoacoustic single from the RBC from a second position, which would provide more information related to the cell orientation. Pulse-echo ultrasound measurements using the same transducers could also be combined with the photoacoustic measurements to help identify the cell orientation, as the ultrasound is sensitive to the cell orientation at the frequencies used [230].

Our technique is highly sensitive to small variations in RBC morphology. A statistically significant difference $(\mathrm{p}<0.001)$ in the spectral slope and amplitude was observed as the osmolality was decreased gradually from 294 to $118 \mathrm{mmol} / \mathrm{kg}$. As the osmolality decreases and the RBC morphology shifts to a spherical shape, the spectral slope and amplitude decrease at each step. The spectrum was similar at 118 and $84 \mathrm{mmol} / \mathrm{kg}$, and no statistical difference was found in the spectral amplitude or slope. This is not surprising, as the RBC is spherical at these osmolalities. This sensitivity to RBC shape variations is unequalled to any kind of existing automated method. In flow cytometry analysis of RBCs, 
light scattering methods could not detect changes in cell morphology until below 170 mosm/kg, at which point the RBCs have already undergone significant swelling [231]. Modern efforts have used light scattering methods to differentiate abnormal RBC morphologies from healthy, but require thousands of measurements and cannot differentiate abnormalities types [232]. Our photoacoustic method is also capable of distinguishing the irregular echinocyte shape from other RBC morphologies. Echinocytes are generally a result of dehydration or high $\mathrm{pH}$; when severely deformed they are termed acanthocytes. Usually observed in vitro, the prevalence in vivo is associated with various diseases such as liver cirrhosis and heart disease, among others [233]. The spectrum has a similar shape to that of nearly spherical RBCs with a spectral minimum around $400 \mathrm{MHz}(147 \mathrm{mmol} / \mathrm{kg}$ in Figure 4.5B), however the echinocytes can be differentiated through quantitative spectral analysis. From 200 to $400 \mathrm{MHz}$, the echinocyte spectral amplitude decreased only half of that of the swelled RBCs, and the standard deviation was higher. Good sensitivity to the morphological shape was observed using a sample size of just 21 RBCs. Automating the procedure to increase the counts by one or two orders or magnitude could give even better sensitivity, and we anticipate we could also differentiate other types of RBC morphological abnormalities such as those brought on by malaria infection (irregular swelling), sickle cell disease (cigarshaped) and stomatocytes (spherically indented), among others.

A significant application of this methodology is for the diagnosis of blood-related diseases. Deviations from the regular biconcave shape are a significant indicator of RBC pathology, whether genetic, infectious or simply from a chemical imbalance. For example, 
spherically shaped RBCs impede blood flow, resulting in anemia, fatigue and splenomegaly [234]. Aggregated RBCs could also be characterized using this system, as the photoacoustic signal changes depending on the type of aggregation [32]. Using a tunable light source would allow for optical spectroscopy measurements using photoacoustics. The absorption of RBCs varies with optical wavelength, which has been exploited to detect oxygenated/deoxygenated blood in vessels [235] and changes in oxygenation due to RBC aggregation [33]. A theoretical framework has been recently developed to detect RBCs infected with malaria using photoacoustics as a function of wavelength [236].

Another significant application is the characterization of donated blood. Blood can be stored up to 42 days after which the blood becomes unsuitable for transfusion. After 7 days in storage, $23 \%$ of RBCs had abnormal shapes such as echinocytes and stomatocytes; after 42 days, it increased to $77 \%$ [237]. Using older blood results in decreased transport efficiency, poor circulation and increasing time spent in hospital. The viability of blood is determined by the storage conditions and how it was prepared. Blood is perishable and demand is constant. There is extensive research devoted to improving the preservation methods by reducing the blood degradation and prolonging the shelf life. A method to rapidly characterize the blood morphology and thus determine its viability does not currently exist and would be useful for rapidly charactering blood both for research purposes, or immediately prior to transfusion [238]. 


\subsection{Conclusions}

Our experiments demonstrate that it is possible to quantify the morphology of RBCs using high-frequency photoacoustic spectral features with a very low sample size. Experimental measurements were validated with numerical simulations using a FEM. The photoacoustic spectral methods described are a first step towards an automated process for the detection of RBC abnormalities via morphological identification with good sensitivity. With some refinement, these methods could eventually be used in a clinical setting to help diagnose RBC pathology using just a single drop of blood. The methods presented are not limited to RBCs; in fact, nearly any micron-sized particle has unique spectral features above $100 \mathrm{MHz}$ that are strongly dependent on the size, shape and orientation, and these features can be used for particle characterization and/or identification. These methods are currently being applied to the analysis of other particles such as biological cells and ultrasound/photoacoustic contrast agents [144, 175].

\subsection{Acknowledgements}

The authors acknowledge helpful discussions and technical support from Eno Hysi and Arthur Worthington (Ryerson University, Canada), Eike Weiss, Wolfgang Bost and Robert Lemor (Kibero GmbH / Fraunhofer Institute, Germany), and Gregory Czarnota (Sunnybrook Health Sciences Center, Canada) for scientific input. The authors thank David Dubins (University of Toronto) for his assistance in measuring the osmolality of our solutions. E. Strohm is supported through a NSERC doctoral fellowship. This research was 
undertaken, in part, thanks to funding from the National Sciences and Engineering Research Council of Canada (216986-2012), the Canadian Institutes of Health Research (CCI 1117964) and the Canada Research Chairs Program awarded to M. Kolios. Funding to purchase the equipment was provided by the Canada Foundation for Innovation, the Ontario Ministry of Research and Innovation, and Ryerson University. 


\section{Chapter 5}

\section{Circulating tumor cell detection using high frequency photoacoustics}

\subsection{Abstract}

A method to detect and differentiate circulating tumor cells (CTCs) from blood cells using photoacoustic frequencies over $100 \mathrm{MHz}$ is presented. At these frequencies, the photoacoustic wavelength is similar to the cell dimensions, resulting in unique features in the photoacoustic signal. Periodically varying minima and maxima occur throughout the photoacoustic power spectrum, where the spacing between minima depends on the ratio of the size to sound speed of the cell. Using a wide-bandwidth transducer with a $375 \mathrm{MHz}$ center frequency and a $532 \mathrm{~nm}$ pulsed laser, the photoacoustic signal was measured from 6 AML cells, 13 melanoma cells and 70 red blood cells (RBCs). The spectral spacing between minima was $85 \pm 27 \mathrm{MHz}$ for melanoma cells, $176 \pm 24 \mathrm{MHz}$ for AML cells, and greater than $230 \mathrm{MHz}$ for RBCs. The photoacoustic signal amplitude normalized to the incident laser energy was $1.1 \pm 0.7 \mathrm{mV} / \mathrm{nJ}$ from melanoma cells, $0.04 \pm 0.01 \mathrm{mV} / \mathrm{nJ}$ from AML cells, and 0.8 $\pm 0.4 \mathrm{mV} / \mathrm{nJ}$ from RBCs. Plotting the normalized signal amplitude vs. spectral spacing 
gives a visual representation of the cell distribution, and allows for grouping of various cell types found in blood to aid in identifying CTCs, particularly melanoma cells which generate a strong photoacoustic signal due to their natural endogenous contrast.

\subsection{Introduction}

Circulating tumor cells (CTCs) are tumor cells that circulate in the bloodstream. They can be shed from a primary tumor in the body, implant elsewhere in the body and result in a secondary tumor. CTCs are common in patients with cancer, but not in healthy people [3]. Tumors can shed cells at early stages of growth prior to detection of the primary tumor [239241]. It may be possible to detect CTCs in the bloodstream as a diagnostic screening method [242]. Lower concentrations of CTCs in blood are related to a favourable outcome in cancer patients [4-9]. Therefore, detection and quantification of CTCs in blood can potentially be used as a screening tool and prognosis estimator to improve patient outcome. There is limited information about the relation between numbers and heterogeneity of CTCs and types of cancers, and the lack of a gold standard for quantifying CTCs remains an issue [10].

Several methods have been developed to detect CTCs from blood samples, including flow cytometry [243, 244], polymerase chain reaction techniques [245], optical based systems [246, 247], enrichment using immunomagnetic beads [248, 249] and photoacoustic detection [250]. Options for in-vivo CTC detection are limited, and include flow cytometry [251, 252] and photoacoustic detection [113, 253-255] methods. The photoacoustic detection of CTCs relies on laser irradiation with the emission of an acoustic wave that can be detected 
using an ultrasound transducer. Optical absorption of energy by the cell is required to generate a photoacoustic signal; this can be endogenous absorbing materials such as hemoglobin (in red blood cells) or melanin (in melanocytes). Detection of circulating melanoma cells is ideal for photoacoustic detection due to their endogenous absorption properties, and several studies have exploited this fact [250, 254, 256-260]. Alternatively, dyes or nanoparticles that are absorbed by specific cells can be added to facilitate photoacoustic wave generation [255, 261-267]. All photoacoustic studies have used transducers with frequencies less than $60 \mathrm{MHz}$, which have been primarily used for image generation.

For cells in the 5-30 $\mu \mathrm{m}$ range, the photoacoustic waves have unique spectral features in the $100-500 \mathrm{MHz}$ range that are directly related to the size and sound speed of the cell [55]. Periodically varying minima and maxima occur throughout the photoacoustic signal power spectrum where the spectral spacing depends directly on the ratio of the cell diameter to sound speed ( $\mathrm{a} / \mathrm{c}$ ratio). As the cell diameter increases, the location and spacing between minima decreases, and as the sound speed increases, the location and spacing between spectral features increases. These spectral features cannot be detected with traditional photoacoustic methods using frequencies under $100 \mathrm{MHz}$.

The photoacoustic spectral spacing and location of spectral minima depend strongly on the cell diameter and sound speed, while the amplitude depends strongly on the absorption properties of the cell; other parameters such as the cell size, sound speed and thermal properties have a lesser effect on the signal amplitude. Variations in the spectral spacing and 
amplitude can be used to aid in identifying and differentiating cells in a sample. In this proof of concept study, we show how the signal photoacoustic signal amplitude and features in the power spectrum can be used to differentiate types of cells in blood, such as red blood cells, CTCs such as melanoma, and hematological malignancies such as acute myeloid leukemia (AML) cells.

\subsection{Method}

\subsubsection{Cell Preparation}

RBCs were extracted from a healthy male volunteer in accordance with the Ryerson Ethics Board (REB \#2012-210). A drop of blood was extracted from the fingertip using a lancet and deposited into one mL of Dulbecco's modified eagle medium (DMEM) for preservation. Two cell lines were used for these experiments, AML-OCI-5 (human acute myeloid leukemia) and B16-F1 (murine melanoma). Cells were incubated inside cell culture flasks with DMEM containing $10 \%$ fetal bovine serum $(\mathrm{FBS})$ at $37^{\circ} \mathrm{C}$ with $5 \% \mathrm{C}_{2}$ and passed every 2-3 days to maintain confluence. AML cells were permeabilized with $0.05 \%$ Triton X-100, and then trypan blue was added as a photoacoustic absorbing agent. Trypan blue was required as the AML cells have negligible absorption at $532 \mathrm{~nm}$. The melanoma cells did not require any treatment as the melanin absorbs visible light.

\subsubsection{Photoacoustic Microscope}

A SASAM acoustic microscope (Kibero $\mathrm{GmbH}$, Germany) was used for all photoacoustic measurements. A transducer was fitted above the sample holder in an IX81 
inverted optical microscope (Olympus, Japan). The transducer and microscope optics were co-aligned. A $532 \mathrm{~nm}$ laser (Teem Photonics, France) was focused through a 10x optical objective onto the sample holder to an approximately $5 \mu \mathrm{m}$ diameter spot, and the transducer aligned to the laser spot. Cells to be measured were moved to a glass bottom dish (Mattek, USA) that was coated with an approximately $200 \mu \mathrm{m}$ thick layer of $1 \%$ agar. The agar was required to reduce back-reflections from the glass substrate and simulated a cell floating in suspension. The sample holder was moved to align a cell within the target area under optical guidance, and photoacoustic measurements made from the cell. The photoacoustic microscope is shown in Figure 5.1A, with an operational schematic in Figure 5.1B. A transducer with a center frequency of $375 \mathrm{MHz}\left(60^{\circ}\right.$ aperture, $42 \%$ bandwidth) was used for all measurements. Laser pulses were generated at $4 \mathrm{kHz}$, and the photoacoustic signals amplified by a $40 \mathrm{~dB}$ amplifier (Miteq, USA), averaged 100 times and digitized at $8 \mathrm{GS} / \mathrm{s}$. Aligning a cell to the target area and recording a measurement takes only seconds per cell. Signal post processing was done manually. The signals measured from directly above the single cells were normalized by removing the transducer response, then a Hamming window applied and the spectrum calculated using the Fast Fourier transform. Further details on the instrument can be found in reference [106], and details on the signal processing methods including normalization can be found in reference [175] or chapter 4.3. 

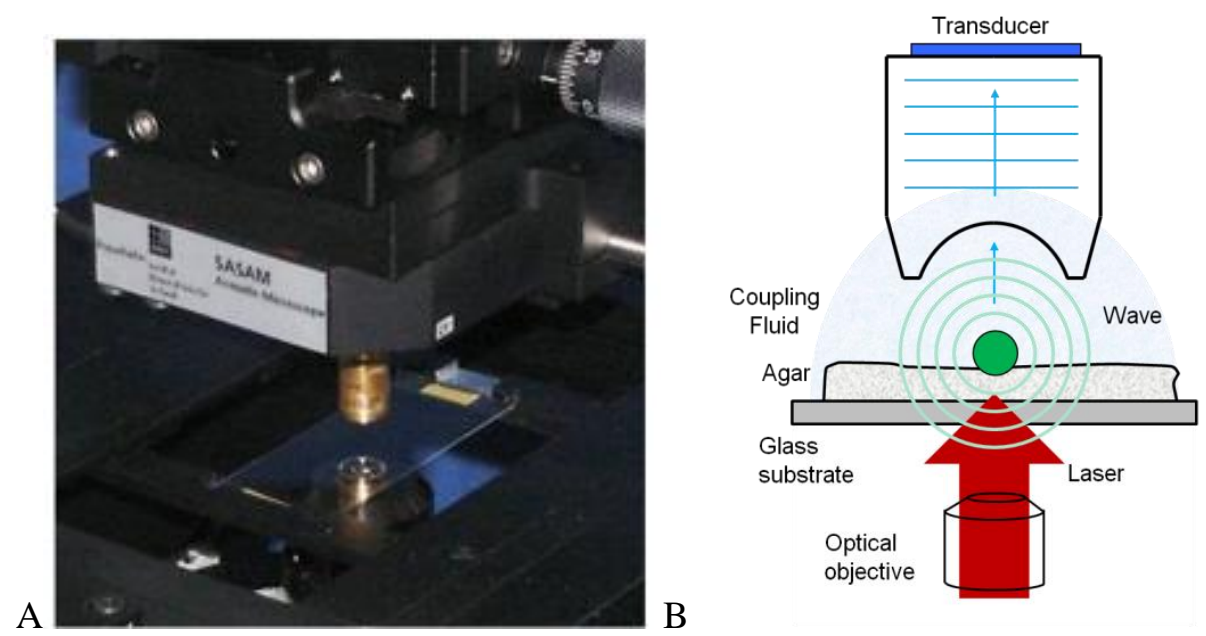

Figure 5.1: The photoacoustic microscope. (A) A picture of the sample holder, with the transducer positioned above the sample and the optics to view the sample and focus the laser are positioned below the sample. (B) A schematic showing the operation of the photoacoustic microscope. The laser is focused onto the cell using an optical objective. The resulting photoacoustic waves are then recorded by the transducer.

\subsection{Results and discussion}

A total of 89 cells were measured: 6 AML, 13 melanoma and 70 RBCs. The diameter of each cell was determined using a calibrated scale and optical imaging, and was $8.6 \pm 1.5$ $\mu \mathrm{m}$ for AML cells, $24.4 \pm 6.8 \mu \mathrm{m}$ for melanoma cells and $7.8 \pm 0.5 \mu \mathrm{m}$ for RBCs. Melanoma cells could potentially be differentiated from other blood cells using optical imaging alone. While most blood cells are $<15 \mu \mathrm{m}$ in diameter, there are some blood cells that can be over $20 \mu \mathrm{m}$ such as monocytes. In addition, non-tumor endothelial cells can also be found in the blood. Photoacoustics is the only method that can be used to distinguish melanoma cells from all other cells in the bloodstream without the use to external additives such as dyes. 
The time domain signals of typical RBCs, melanoma and AML cells are shown in Figure 5.2. Figure 5.3 shows the power spectrum for these four cells. Distinct spectral features that can differentiate the cells are now evident. The two RBC orientations have minor variations throughout the spectra, with the vertically oriented RBC displaying two spectral minima around 200 and $500 \mathrm{MHz}$. In contrast, the melanoma cell has six spectral minima spaced $57 \pm 2 \mathrm{MHz}$ apart, and the AML cell has three minima spaced $121 \pm 15 \mathrm{MHz}$ apart. The vertically oriented $\mathrm{RBC}$ had a $\Delta \mathrm{f}$ of $300 \mathrm{MHz}$, while spectral minima were not observed with the horizontal RBCs. The spectral spacing $\Delta \mathrm{f}$ was calculated for 6 AML cells, 13 melanoma cells and 70 RBCs (the RBCs were in various orientations). Spectral minima were absent in horizontally oriented RBCs, therefore $\Delta$ f was set to $400 \mathrm{MHz}$.

Another measured property that can be used for cell differentiation is the photoacoustic signal amplitude, which is proportional to the absorption coefficient of the cell and the laser energy. The signal amplitudes for RBCs were 70-90 mV using a laser energy of $25 \mathrm{~nJ} /$ pulse, melanoma cells $20-50 \mathrm{mV}$ for a laser energy of $25 \mathrm{~nJ} /$ pulse and AML cells 5-10 $\mathrm{mV}$ for a laser energy of $220 \mathrm{~nJ} / \mathrm{pulse}$. The photoacoustic signal was normalized to the laser energy (units of $\mathrm{mV} / \mathrm{nJ}$ ) so all measurements could be directly compared; the normalized photoacoustic signal was $0.04 \pm 0.01 \mathrm{mV} / \mathrm{nJ}$ for $\mathrm{AML}$ cells, $1.1 \pm 0.7 \mathrm{mV} / \mathrm{nJ}$ for melanoma cells and $0.8 \pm 0.4 \mathrm{mV} / \mathrm{nJ}$ for RBCs. The spectral spacing, normalized photoacoustic signal amplitude and the optical diameter are summarized in Table 5-1.

A plot of the normalized signal amplitude vs. $\Delta \mathrm{f}$ is shown in Figure 5.4. Each cell type can be identified according to its location on the chart. RBCs are located in the upper 
right area, melanoma cells in the upper left and AML cells in the bottom left. The melanoma cells and RBCs both have large signal amplitudes, however they can easily be differentiated as the melanoma cells have a much smaller $\Delta \mathrm{f}$. Unlike the melanoma and RBCs, AML cells have no endogenous contrast; dye was added to facilitate the photoacoustic signal emission. For these cells, Trypan blue was used after cell membrane permeabilization. When targeting cancer cells, nanoparticles or dyes that bind preferentially to tumor cells could be used instead of Trypan blue; this dye was used to simulate the selective targeting of a tumor cells. For example, nanoparticles conjugated with the EPCAM antibody could be added that only attach to circulating endothelial tumor cells that commonly express this antibody [268]. The photoacoustic signal generated depends directly on the dye/nanoparticle concentration within the cell. Therefore the concentration must be adjusted to achieve good separation from other cells, and be constant between measurements for consistent results.

An advantage of using the spectral characteristics of the photoacoustic signal instead of other methods such as optical spectroscopy or conventional photoacoustic spectroscopy (which measures the photoacoustic signal amplitude as a function of the optical excitation wavelength) is the extra information extracted from the photoacoustic spectrum. Only one measurement is required per cell instead of sweeping over numerous optical wavelengths. Moreover, ultrasound pulse echo measurements could be combined with the photoacoustic measurements as the ultrasound backscatter spectrum also contains periodically varying minima and maxima that depend on the cell size and sound speed [118, 123, 125, 269, 270]. Our method could be improved by incorporating the ultrasound backscatter spectrum in 
addition to the photoacoustic signals. Optical spectroscopy methods using multiple laser wavelength illuminations could be combined with the spectral analysis to target specific chromophores in a cell, such as DNA, granules in WBCs, or dyeing of the cytoplasmic region.

Despite the small number of cells measured for this proof of principle study, good differentiation between cell types has been observed. For clinical use, a very large number of cells would have to be measured. This methodology could be translated to a flow device incorporating a laser and ultrasound transducer to record the signals from thousands of cells, increasing the accuracy of the measurement.
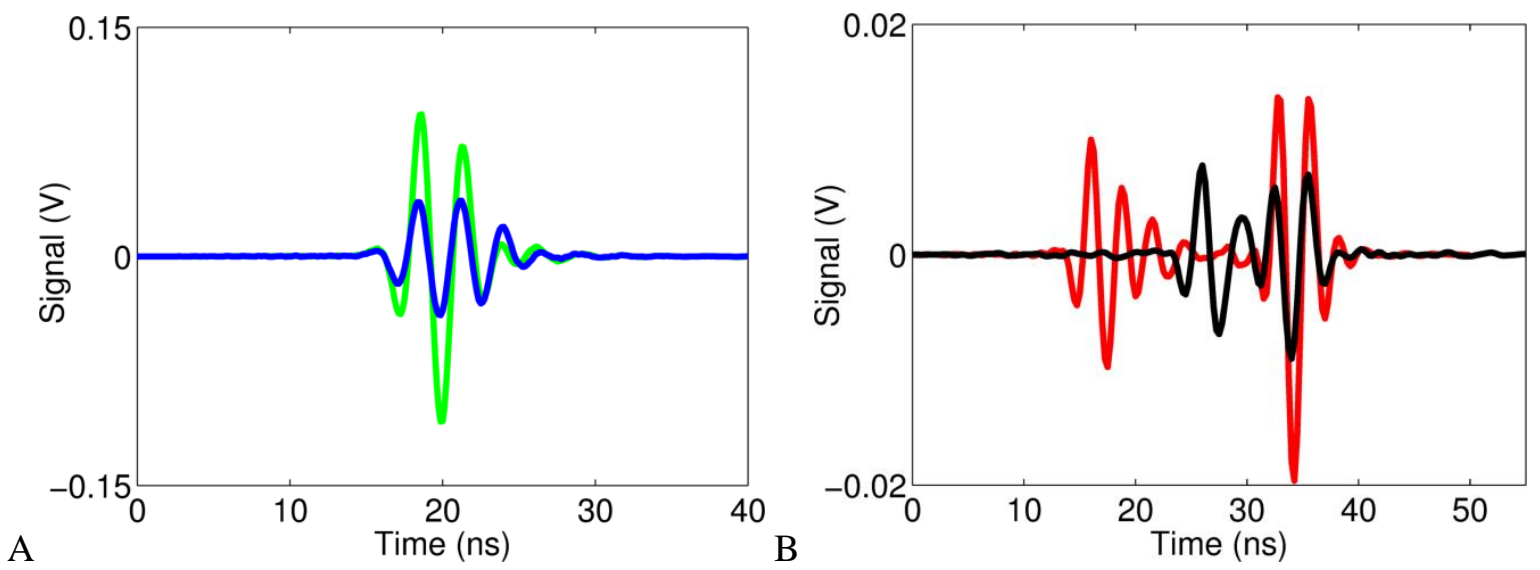

Figure 5.2: The time domain signals recorded from (A) RBCs in a horizontal (green) and vertical (blue) orientation, and (B) melanoma cells (red) and AML cells (black). 

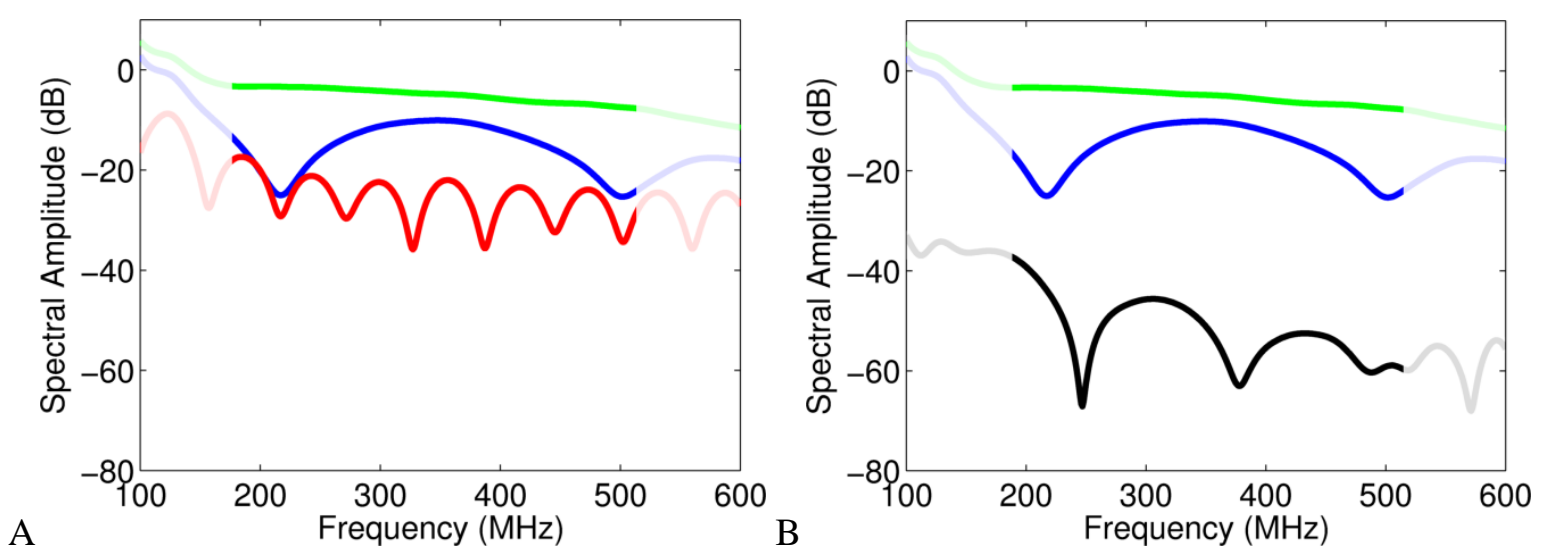

Figure 5.3: Typical photoacoustic power spectra from cells. The spectrum of melanoma cells (A, red) and AML cells (B, black) compared to RBCs measured in a horizontal (green) and vertical (blue) orientation. The area outside of the transducer bandwidth has been grayed.

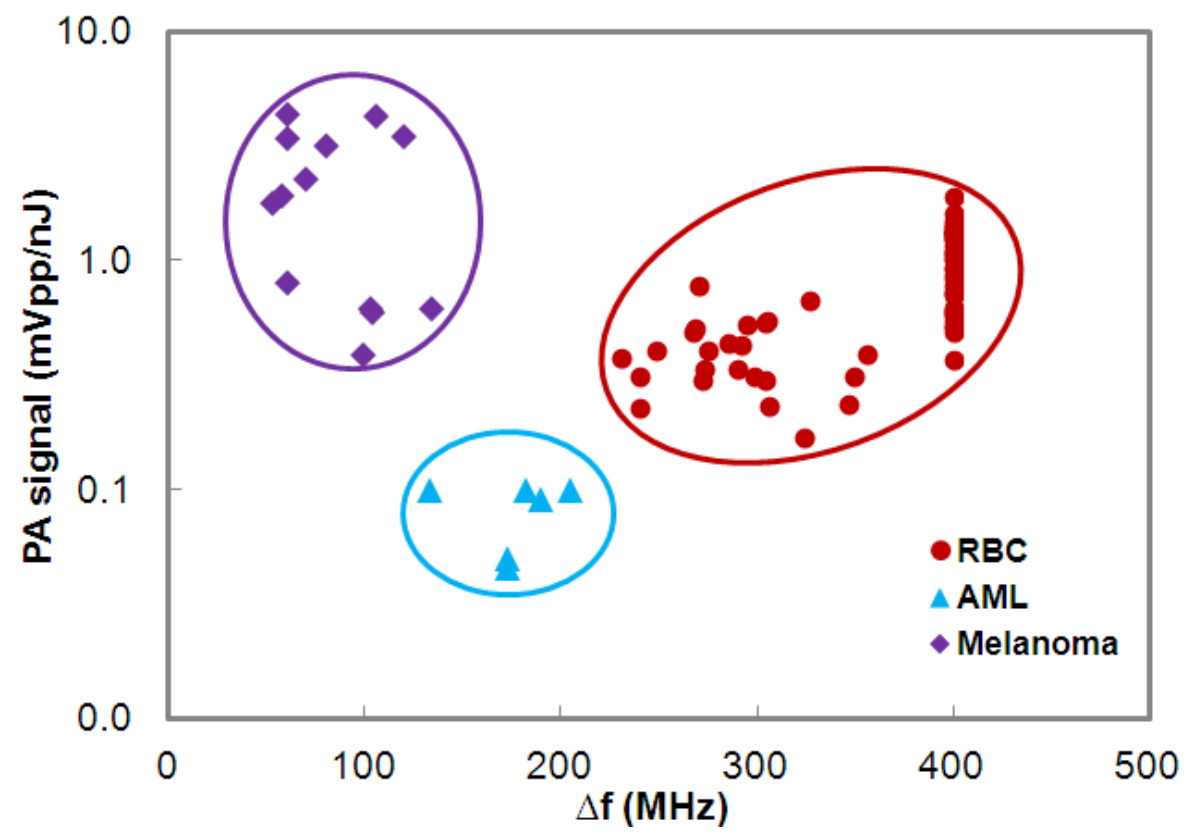

Figure 5.4: The measured photoacoustic signal (normalized to laser energy) vs. the photoacoustic spectral spacing $\Delta f$. The location of the cells in this graph can be used to identify them. 


\begin{tabular}{ccccc}
\hline Cell & Number of cells & $\begin{array}{c}\text { Diameter } \\
(\boldsymbol{\mu m})\end{array}$ & $\begin{array}{c}\text { Spectral Spacing } \\
\boldsymbol{\Delta} \boldsymbol{f}(\mathbf{M H z})\end{array}$ & $\begin{array}{c}\text { Normalized Signal } \\
\mathbf{a m p l i t u d e}\left(\mathbf{m V}_{\mathbf{p p}} / \mathbf{n} \mathbf{J}\right)\end{array}$ \\
\hline AML & 6 & $8.6 \pm 1.5$ & $176 \pm 24$ & $0.04 \pm 0.01$ \\
Melanoma & 13 & $24.4 \pm 6.8$ & $85 \pm 27$ & $1.1 \pm 0.7$ \\
RBC & 70 & $7.8 \pm 0.5$ & $230-400$ & $0.8 \pm 0.4$ \\
\hline
\end{tabular}

Table 5-1: A summary of the diameter (measured optically), spectral spacing $\Delta f$ and normalized signal amplitude for the different cell types.

\subsection{Conclusions}

This study has demonstrated that purely photoacoustic methods based on the signal amplitude and spectral features can be used to differentiate types of cells found in blood. In particular, CTCs such as melanoma cells can be detected using label-free methods. Other tumor cells can also be detected by using specific dyes or nanoparticles that preferentially bind to tumor cells and not healthy cells. This work could be translated to a flow device that could measure thousands of cells per second, enabling measurement of a large sample size to detect rare tumor cells. 


\section{Chapter 6}

\section{Conclusions and future work}

\subsection{Summary of findings}

The work described in this dissertation describes the first theoretical and experimental photoacoustic measurements of single biological cells using frequencies over $100 \mathrm{MHz}$. At these frequencies, the wavelength is similar to the dimensions of the cell, resulting in signals that are rich in information. This section summarizes the significant findings and conclusions from chapters 2 through 5 .

\subsubsection{Photoacoustic numerical method development (chapter 2)}

Analytical solutions for the photoacoustic signals from spherical particles were first developed in the late 1980 's, and were validated using mm-sized particles and photoacoustic frequencies well under $100 \mathrm{MHz}$. These theories have never been used for micron-sized particles; this chapter was devoted to investigating the theoretical and experimental photoacoustic signals from micron-sized PFC droplets. These droplets are ideal particles for a photoacoustic analysis, as they are spherical, homogeneous and nearly optically transparent, ensuring the entire particle volume is irradiated with equal laser intensity. In 
addition, the size of the droplets (roughly 1-20 $\mu \mathrm{m}$ ) ensure that unique spectral patterns exist using photoacoustic frequencies between 100-1000 MHz. A FEM was developed to obtain the spectral patterns from particles where the analytical solution cannot be used.

Photoacoustic measurements were made of a single $3.1 \mu \mathrm{m}$ diameter PFC droplet in suspension, and compared to the analytical solution (equation 2-2). Excellent agreement in the location of the spectral minima and maxima were found between theory and measurements using a $375 \mathrm{MHz}$ transducer and the known droplet parameters (diameter =

$3.1 \mu \mathrm{m}$, sound speed $=480 \mathrm{~m} / \mathrm{s}$, density $=1650 \mathrm{~kg} / \mathrm{m}^{3}$ ). A FEM was solved using these same parameters. The FEM results agreed with both the analytical solution and measurements, validating the FEM.

The analytical model and FEM were then applied to measurements of droplets on top of a glass substrate ranging in diameter from $2.8-3.6 \mu \mathrm{m}$. This substrate strongly reflected the acoustic waves travelling down from the droplet back towards the transducer, interfering with the waves traveling towards the transducer from the top of the droplet. In this situation, extra spectral minima and maxima were observed throughout the power spectrum. The FEM accurately reproduced these extra spectral features, while the analytical solution did not.

\subsubsection{Model validation for ideal photoacoustic particles (chapter 3)}

This chapter further investigates the photoacoustic signals from single PFC droplets to examine how the spectral features can be used to determine particle parameters. The signal from ultra-wide bandwidth transducers (from 300-1000 MHz) were used to record the 
photoacoustic signals. Through this frequency range, dispersion increases the sound speed of the PFC liquid by a small but measureable amount (480 to $486 \mathrm{~m} / \mathrm{s}$ from 1 to $1000 \mathrm{MHz}$ ), affecting the location of the spectral minima and maxima. The analytical solution was modified to account for changes in the sound speed as a function of frequency, resulting in the spectral minima and maxima scaling to higher frequencies as the frequency increased. Using this modified model, the droplets were measured using two different transducers. A $5.7 \mu \mathrm{m}$ droplet was measured using a $375 \mathrm{MHz}$ transducer (bandwidth approximately 150 to $450 \mathrm{MHz}$ ), and a $2.45 \mu \mathrm{m}$ and $6.95 \mu \mathrm{m}$ droplet were measured using a $750 \mathrm{MHz}$ transducer (bandwidth approximately 400-900 MHz). Excellent agreement in the location of the spectral features between theory and measured were observed in these three cases for the known droplet parameters (sound speed $=480$ to $486 \mathrm{~m} / \mathrm{s}$, density $=1650 \mathrm{~kg} / \mathrm{m}^{3}$ ).

The sound speed for the droplets is known, and the other variable that affects the location of the spectral minima is the diameter. The diameter of the droplet can be found by varying the diameter in the model until the location of the spectral minima match with the measured spectra. A total of 18 droplets were measured, and the droplet extracted from the spectrum was compared to three other independent measurements of diameter: time of flight using the photoacoustic time domain signal and ultrasound time domain signal, and direct optical measurements. Excellent agreement in the diameter was observed for all four methods. 


\subsubsection{Application towards irregularly shaped RBCs (chapter 4)}

A preliminary investigation into the photoacoustic signals generated from single RBCs is described in this chapter. RBCs have been frequently studied using photoacoustic frequencies below $100 \mathrm{MHz}$; however this is the first study using frequencies over $100 \mathrm{MHz}$. The size, orientation and shape significantly affect the shape of the spectrum for frequencies over $100 \mathrm{MHz}$.

The FEM developed in chapter 2 was used to investigate how the size and orientation affect the photoacoustic spectrum. When a RBC was oriented horizontally (thickness is about $1 \mu \mathrm{m}$ ), a single spectral minima is observed around $800 \mathrm{MHz}$; this location depends on the thickness of the RBC. When a RBC was oriented vertically (diameter of the cell ranges from about 7-8.5 $\mu \mathrm{m}$ ), regular spectral minima were observed with a frequency spacing of about $300 \mathrm{MHz}$. As the diameter increased from $6.8 \mu \mathrm{m}$ to $8.8 \mu \mathrm{m}$, the frequency spacing decreased from about $350 \mathrm{MHz}$ to $200 \mathrm{MHz}$, respectively. This indicates that provided the $\mathrm{RBC}$ orientation is known, the RBC diameter could be determined from the location of the spectral minima. When a RBC was oriented horizontally, a single spectral minimum was observed around $800 \mathrm{MHz}$, with the exact location depending on the RBC thickness. As the RBC rotated from a vertical to horizontal orientation (relative to the transducer), the spectral minima shift to higher frequencies. These numerical simulations were compared to measurements of a single RBC initially in a vertical orientation, and gradually tipped over to a horizontal orientation. A gradual increase in the spectra minimum was observed, agreeing with the numerical simulations. 
Practical applications were investigated using the photoacoustic spectrum. $21 \mathrm{RBCs}$ were measured in osmolalities ranging from $294 \mathrm{mmol} / \mathrm{kg}$ (isotonic) to $84 \mathrm{mmol} / \mathrm{kg}$. As the osmolality decreased, the RBCs swelled by taking in water; the RBC shape gradually shifted from the normal biconcave shape (at $294 \mathrm{mmol} / \mathrm{kg}$ ) to a spherical shape at $84 \mathrm{mmol} / \mathrm{kg}$. As the RBCs swelled, the average photoacoustic spectrum from the $21 \mathrm{RBCs}$ changed. A statistically significant decrease in the spectral amplitude (at $288 \mathrm{MHz}$ ) and spectral slope (from 275-300 MHz) was observed at each drop in osmolality ( $\mathrm{p}<0.001$ ) except from 118 to $84 \mathrm{mmol} / \mathrm{kg}$; at these osmolalities, the RBCs was already spherical. The photoacoustic signal from echinocytes was also investigated. Healthy RBCs with a biconcave shape were measured in medium. The RBCs were then immersed in PBS for 24 hours, depleting the ATP energy within the cell. This results in an irregularly shaped RBC with specular protrusions located over the surface. The spectrum from the echinocytes was significantly different than the healthy RBCs initially measured. The echinocytes were then immersed in medium, where they regained their normal biconcave shape. The spectrum measured from these cells was very similar to the first measurements of healthy RBCs, indicating that the echinocytes can regain their normal biconcave shape under some circumstances. These high-frequency photoacoustic measurements are highly sensitive to changes in RBC morphology.

\subsubsection{Cell differentiation and identification (chapter 5)}

A method to identify and differentiate cell types based on their spectral features and signal amplitude was presented in chapter 5 . When the photoacoustic frequency is in the 100$500 \mathrm{MHz}$ range, the photoacoustic wavelength is similar to the size of a single cell; this 
results in periodically varying minima and maxima that occur throughout the power spectrum. The frequency spacing between the spectral minima is directly related to the ratio of the size to sound speed of the cell. Three types of cells were investigated: $70 \mathrm{RBCs}, 13$ melanoma cells and 6 AML cells. To facilitate photoacoustic signals from AML cells, they were permeabilized with triton X-100, then trypan blue added. The spectral spacing between minima was $85 \pm 27 \mathrm{MHz}$ for melanoma cells, $176 \pm 24 \mathrm{MHz}$ for AML cells, and greater than $230 \mathrm{MHz}$ for RBCs. The photoacoustic signal amplitude normalized to the incident laser energy was $1.1 \pm 0.7 \mathrm{mV} / \mathrm{nJ}$ from melanoma cells, $0.04 \pm 0.01 \mathrm{mV} / \mathrm{nJ}$ from AML cells, and $0.8 \pm 0.4 \mathrm{mV} / \mathrm{nJ}$ from RBCs. When plotted on a graph of photoacoustic signal vs. spectral spacing, three groups were observed; one group for each type of cell. These methods could be used to differentiate and count RBCs from leukocytes, or identify specific cells in a sample such a CTCs.

\subsection{Clinical significance}

There are many applications where photoacoustic measurements of single cells could be used in a clinical setting. Due to the strong attenuation using frequencies above $100 \mathrm{MHz}$, in-vivo applications are limited. Manual measurements using a photoacoustic microscope (as discussed in this dissertation) are restricted to small sample sizes only, and a large cell count is typically required for a diagnosis. All measurements have examined cells in suspension, or loosely bound to a substrate; these techniques could be translated to a flow device that is capable of performing large numbers of measurements in a short period of time (see section 
6.3, future work).

Analysis of blood samples are ideal for high-frequency photoacoustic measurements, as outlined in chapters 4 and 5. RBCs generate strong photoacoustic signals using endogenous contrast which are dependent on their size and morphology. Disease and infection can alter their size and morphology. The gold standard for blood cell analysis is visual inspection of a blood smear under an optical microscope by a technician. Automated methods such as hematology analyzers can size and count RBCs use light scattering and electrical impedance methods. These devices infer the volume of the cell, and then estimate the size and shape from the volume. These devices can typically detect if a sample is outside of normal guidelines, but cannot determine what the abnormality is. We have demonstrated that high frequency photoacoustic measurements are highly sensitive to changes in $\mathrm{RBC}$ morphology; this could potentially be used to diagnose pathology with better sensitivity than current methods.

These methods can also be applied to measurements of WBCs. While WBCs have negligible optical absorption in the visible spectrum, exogenous contrast agents such as dyes and/or nanoparticles can be used to facilitate the generation of photoacoustic waves from these cells. Preliminary work shown in chapter 5 demonstrates that RBCs have a distinctly different photoacoustic spectrum compared to other cells (such as melanoma and AML cells). The various WBC sub-types such as neutrophils, eosinophils and basophils all have unique size and microstructure that can be used to count and identify them using dyes (such as Wright-Giemsa) and visual inspection [206, 271]. These methods cannot be used to 
identify CTCs; fluorescence methods such as flow cytometry with expensive dyes are typically used. Our preliminary work has shown that different cells in blood could be differentiated using the photoacoustic amplitude and spectral spacing (chapter 5). By using dyes or nanoparticles that preferentially bind to tumor cells, CTCs could be detected using photoacoustics without fluorescence. The RBCs, WBCs and tumor cells could all be detected and counted using one method.

Early detection of cancer is crucial for survival, and the methods described in this dissertation could be eventually applied in a clinical setting. To achieve clinically relevant results, samples would have to be examined in an automated device with minimal user intervention. The next section describes the future work devoted to realizing this significant next step.

\subsection{Future work}

The basic proof of principle methods of cell identification and classification have been outlined in this dissertation. The next goal is translating this methods into a highthroughput system for an automated analysis of a sample. A microfluidic device that is capable of streaming cells sequentially through a target area where they are irradiated by a laser and/or ultrasound is currently being designed (Figure 6.1). This would enable thousands of measurements within seconds. This dissertation has concentrated on photoacoustic measurements of single cells; pulse echo ultrasound can be easily integrated due to the complementary nature of ultrasound and photoacoustics. Ultrasound echoes from single cells 
exhibit unique spectral features which are similar to the photoacoustic spectrum $[118,123$, $125,269,270]$; this would add a secondary method of cell characterization in addition to photoacoustic measurements.

The photoacoustic microscope was modified to enable simultaneous emission of the laser and ultrasound pulse. Figure 6.2 shows the combined photoacoustic and ultrasound measurements of a single cell. The photoacoustic signal is measured first (at $750 \mathrm{~ns}$ ), then the signal resulting from direct laser irradiation of the transducer (1100 ns), followed by noise within the transducer (1200-1400 ns), then the ultrasound signal at $1650 \mathrm{~ns}$.

Basic design of the flow device is in progress. Microfluidic devices are commonly used to focus particles into a narrow stream for analysis. However, most devices focus in 2D through a narrow channel that is typically less than $100 \mu \mathrm{m}$. 3D-focusing microfluidic devices are less common and structurally complex. They are difficult to fabricate using traditional Polydimethylsiloxane (PDMS), requiring several lithography and molding cycles [272]. Our device requires channels approximately $1 \times 1 \mathrm{~mm}$, but very few PDMS devices have been made with such large dimensions due to time and expense constraints. We have designed a basic 2D millifluidic device with mm-sized channels using Poly(methyl methacrylate) (PMMA, also known as acrylic or Plexiglas). This material can be laser cut to specifications and the layers bonded together. The 2D prototype designed is shown in figure Figure 6.3; it consists of three inlets (two for sheath flow and one for the cell fluid) and one outlet. The sheath flow is much higher than the cell flow; the cells are focused to the center of the channel. The position of the cell flow can be moved within the channel by adjusting 
the flow ratio of the two sheath flows. This device can only focus cells in $2 \mathrm{D}$; this is sufficient for many lab-on-a-chip designs, but not in cases where axial focusing is also required in addition to lateral focusing. The $2 \mathrm{D}$ prototype has been designed to test the flow characteristics, and examine how the transducer and laser will be integrated into the device. A 3D device will then be designed to ensure the cells are centered in the device. Combining a 3D-flow device with photoacoustic and ultrasound measurements would enable a new type of instrument with advantages over currently accepted diagnostic devices such as the Coulter counter and flow cytometers.

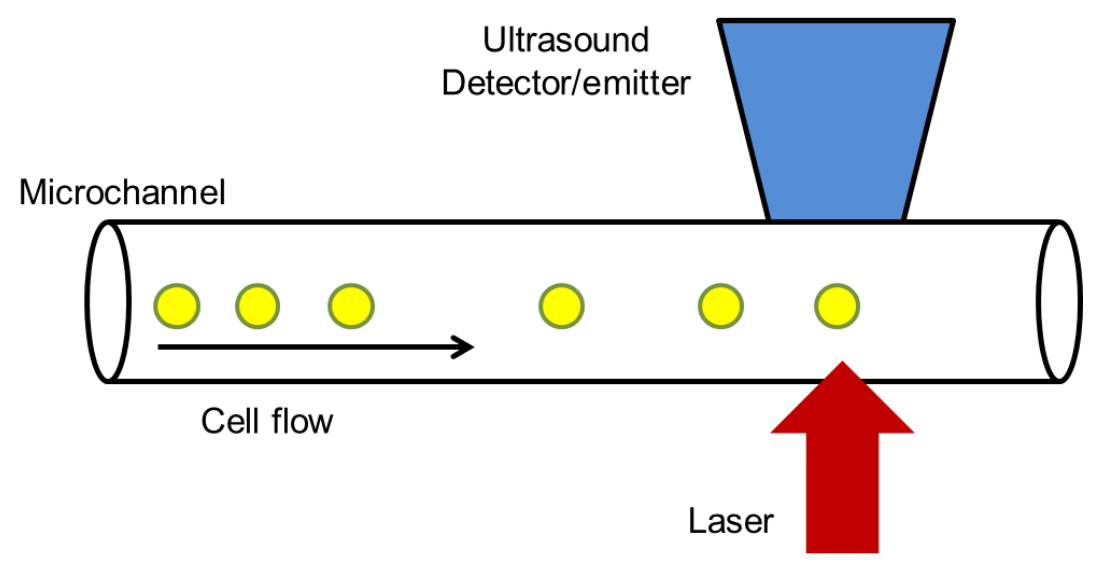

Figure 6.1: Basic schematic of the flow device showing the particle flow through the PAUS target area. 


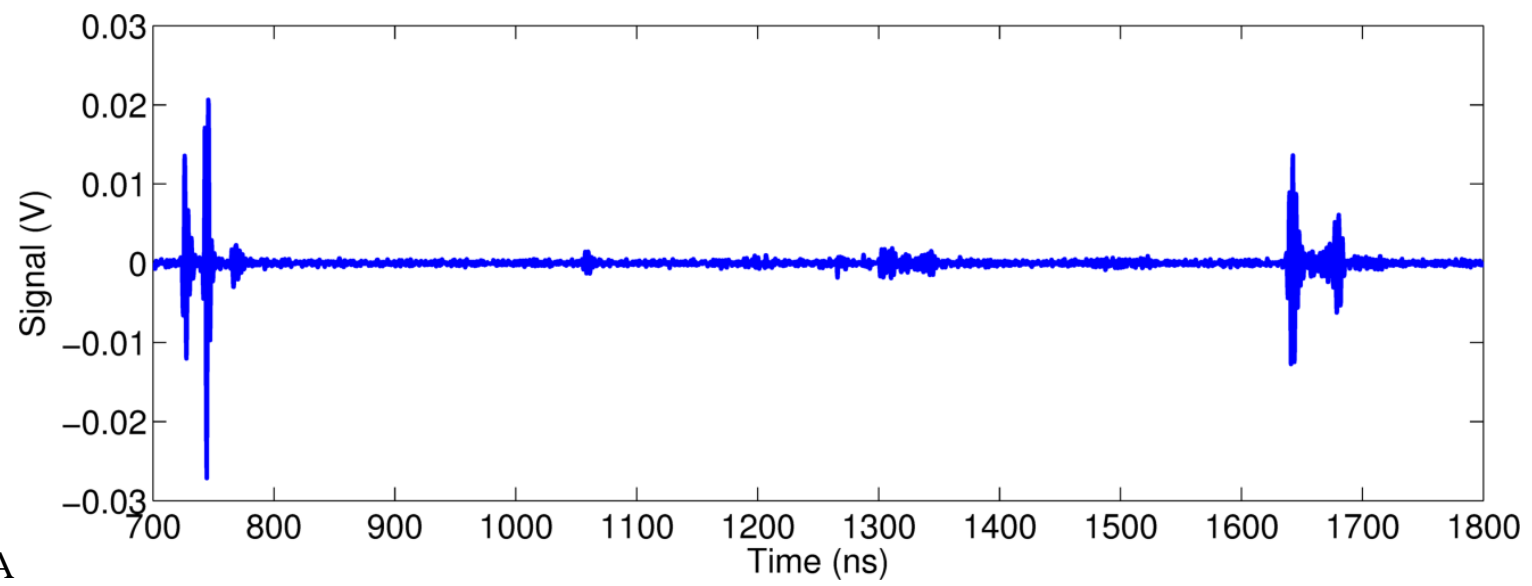

A
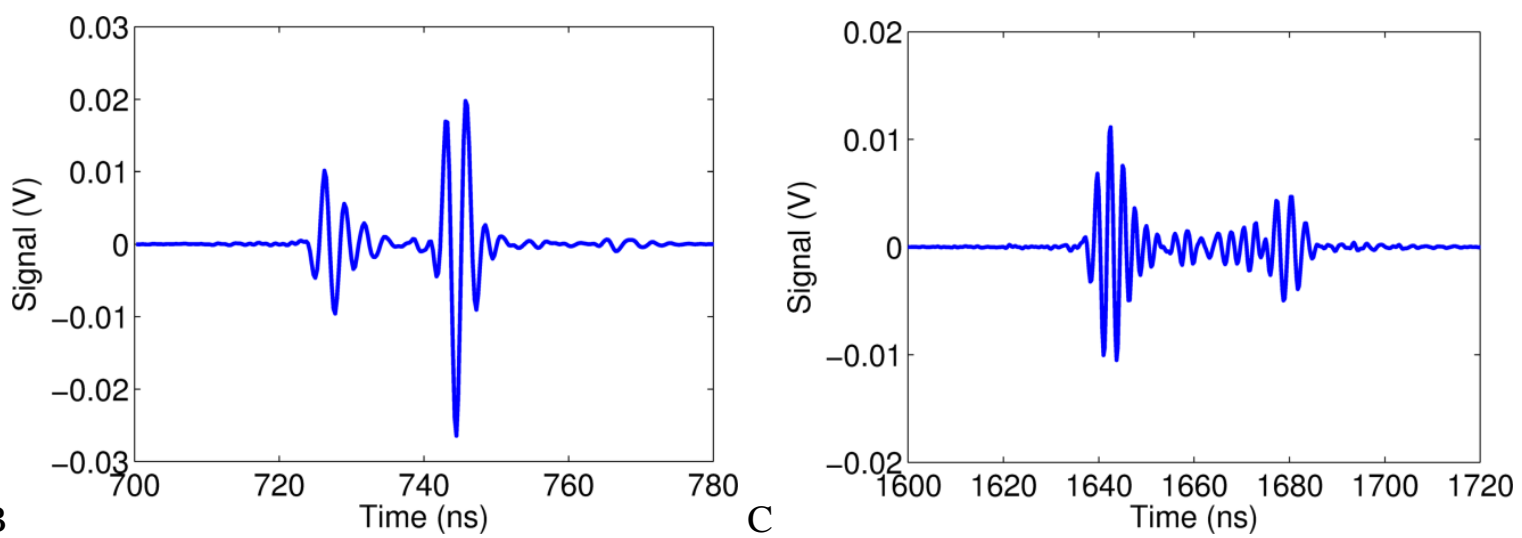

Figure 6.2: Simultaneous measurements of a single cell using photoacoustics and ultrasound. (A) Recorded a-scan showing both the photoacoustic and ultrasound signal, and zoomed image of the photoacoustic signal (B) and the ultrasound signal (C). 

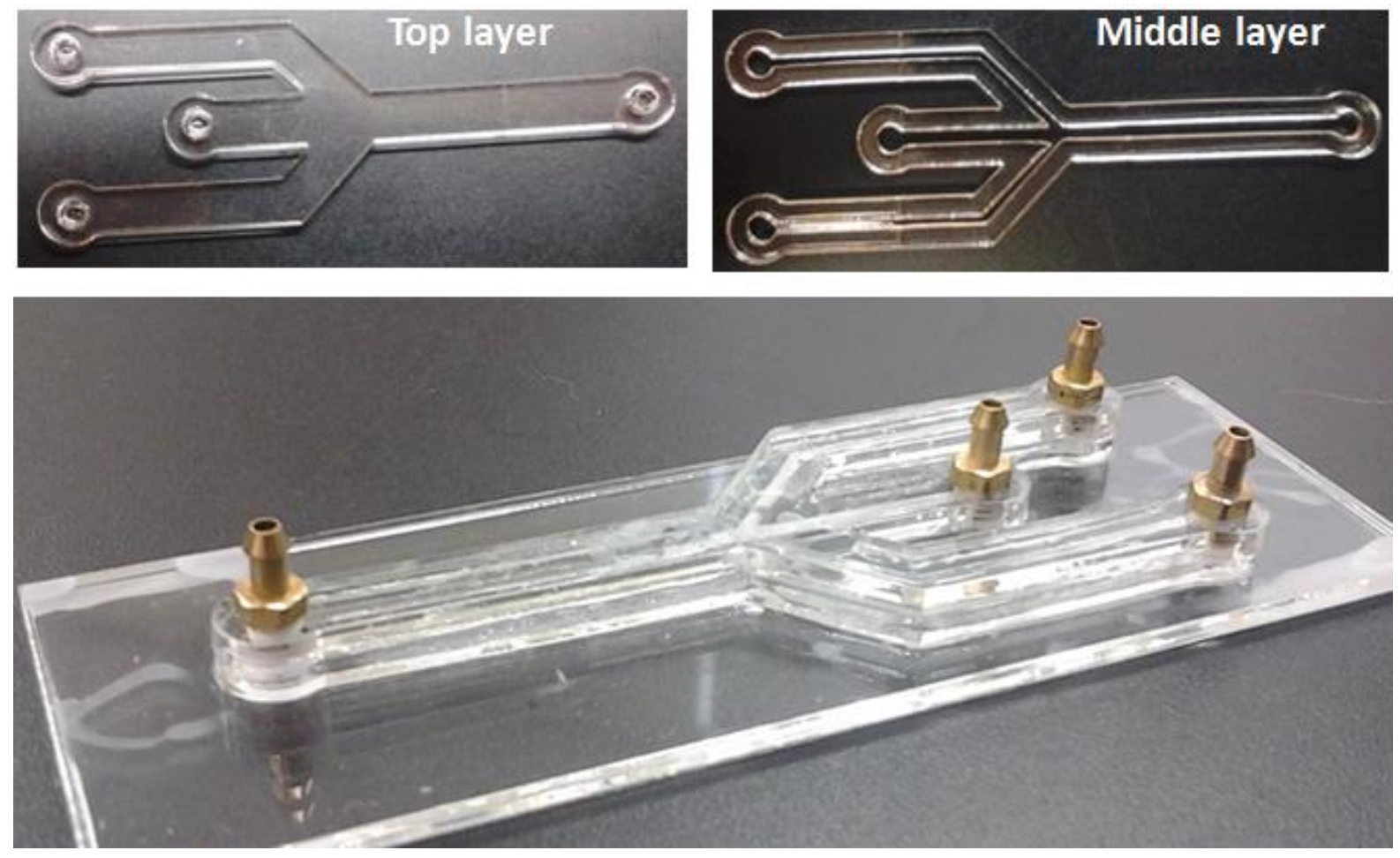

Figure 6.3: The millifluidic device. The top layer (A) is placed on top of the middle layer (B), which has grooves cut into the plastic for particle flow. (C) The layers are glued together to create a 3D structure. Tubes are attached to the nozzles for fluid flow. The size of the bottom structure is $25 \times 75$ $\mathrm{mm}$.
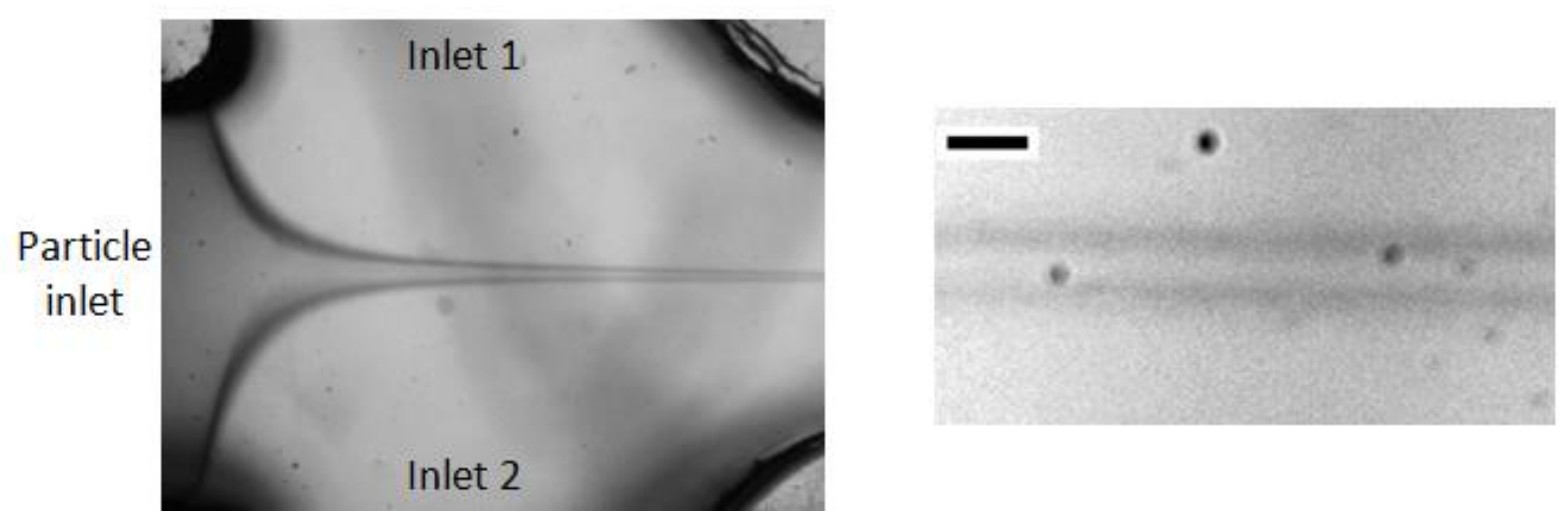

Figure 6.4: Flow focusing performance of the microfluidic device at the inlet/mixing area (left) and optical view of the focused stream at the observation region (right). The particles are confined to a 15 $\mu \mathrm{m}$ width. The scale bar is $20 \mu \mathrm{m}$. 


\subsection{Conclusions}

The findings presented in this dissertation describe the first theoretical and experimental photoacoustic measurements of micron-sized particles and cells using frequencies over $100 \mathrm{MHz}$. These preliminary studies are the first practical step towards using ultra-high frequency sound waves for identifying and differentiating particles and cells in a sample. Clinical applications such as identification of infection and disease through changes in cell morphology and/or physical properties, and detection of CTCs in blood using high speed microfluidic systems have been proposed and will be investigated as future work. 


\section{Appendix A}

\section{Publications}

A summary of publications directly and indirectly related to this dissertation since beginning the $\mathrm{PhD}$ program in 2010.

\section{Book chapters (1)}

1. E.M. Strohm, G. Czarnota, M.C. Kolios, "Acoustic microscopy of cells" in Quantitative Ultrasound of Soft Tissue, J. Mamou and M. Oelze (Eds.). Springer, 2013

\section{Publications in refereed journals (4)}

1. E.M. Strohm, E.S.L. Berndl, and M.C. Kolios, "High frequency label-free photoacoustic microscopy of single cells," Photoacoustics, 1(3-4), 2013, pp. 49-53.

2. E.M. Strohm, E. Berndl, M.C. Kolios, "Probing red blood cell morphology using high frequency photoacoustics," Biophysical Journal, 105(1), 2013, pp. 59-67.

**This publication was covered by journalists and featured in Scientific American, New Scientist, Nature Highlights and ScienceNOW.

3. E.M. Strohm, I. Gorelikov, N. Matsuura, M.C. Kolios, "Acoustic and photoacoustic characterization of micron-sized perfluorocarbon emulsions", Journal of Biomedical 
Optics, 17(9), 2012, pp. 096016-26.

4. E.M. Strohm, M. Rui, I. Gorelikov, N. Matsuura, M.C. Kolios, "Vaporization of perfluorocarbon droplets using optical irradiation", Biomedical Optics Express, 2(6), 2011, pp. 1432-1442.

\section{Publications in conference proceedings (15)}

1. E.M. Strohm, E. Berndl, M.C. Kolios, "Circulating tumor cell detection using photoacoustic spectral methods", SPIE Proceedings 2014.

2. Y.J. Wang, E.M. Strohm, Y. Sun, C. Niu, Y. Zheng, Z. Wang, M.C. Kolios, "PLGA/PFC particles loaded with gold nanoparticles as dual contrast agents for photoacoustic and ultrasound imaging", SPIE Proceedings 2014.

3. F. Kibria, E. Hysi, E.M. Strohm, M.C. Kolios, "Identification of red blood cell rouleaux formation using photoacoustic ultrasound spectroscopy”, SPIE Proceedings 2014.

4. Y. Sun, C. Niu, Y. Wang, E.M. Strohm, H. Ran, Y. Zheng, Z. Wang, and M.C. Kolios, "Vaporization, Photoacoustic and Acoustic characterization of PLGA/PFH particles loaded with optically absorbing materials," in IEEE International Ultrasonics Symposium, Prague, Czech Republic, July 2013.

5. E.M. Strohm, and M.C. Kolios. "Sound Speed Estimation in Single Cells Using the Ultrasound Backscatter Power Spectrum." In ICA 2013 Montreal, 19:075012. Montreal, Canada: Acoustical Society of America, 2013.

6. E.S. Berndl, L. Wirtzfeld, E.M. Strohm, M.C. Kolios. "Acoustical Imaging of Internal Spheroid Structures for a Series of Frequencies." In ICA 2013 Montreal, 19:075086. Montreal, Canada: Acoustical Society of America, 2013.

7. M.C. Kolios, E.S. Berndl, L. Wirtzfeld, E.M. Strohm, and G.J. Czarnota. "Acoustic and 
Photoacoustic Imaging of Spheroids.” In ICA 2013 Montreal, 19:075075. Montreal, Canada: Acoustical Society of America, 2013.

8. M.M. Pasternak, E.M. Strohm, and M.C. Kolios. "An Analysis of the Acoustic Properties of the Cell Cycle and Apoptosis in MCF-7 Cells." In ICA 2013 Montreal, 19:075014. Montreal, Canada: Acoustical Society of America, 2013.

9. E.M. Strohm, E. Berndl, M.C. Kolios, "A photoacoustic technique to measure the properties of single cells", SPIE Proceedings 2013.

10. E.M. Strohm, E. Hysi, M.C. Kolios, "Photoacoustic measurements of single red blood cells" Proceedings of the 2012 IEEE International Ultrasonics Symposium.

11. E.M. Strohm, I. Gorelikov, N. Matsuura, M.C. Kolios, "Photoacoustic spectral characterization perfluorocarbon droplets", SPIE Proceedings 2012.

12. E.M. Strohm and M.C. Kolios, "Sound velocity and attenuation measurements of perfluorocarbon droplets using photoacoustic methods", Proceedings of the 2011 IEEE International Ultrasonics Symposium.

13. E.M. Strohm, M. Rui, I. Gorelikov, N. Matsuura, M.C. Kolios, "Photoacoustic characterization of micron-sized perfluorocarbon droplets", SPIE Proceedings 2011.

14. E.M. Strohm, I. Gorelikov, N. Matsuura, M.C. Kolios, "Optical droplet vaporization: photoacoustic characterization of micron-sized perfluorocarbon droplets", Proceedings of the 2010 IEEE International Ultrasonics Symposium.

15. E.M. Strohm, M. Rui, M. Pasternak, M. Mercado, G. Czarnota, M.C. Kolios, "A comparison of the ultrasonic properties of cells during apoptosis and mitosis using acoustic microscopy", Proceedings of the 2010 IEEE International Ultrasonics Symposium. 


\section{Bibliography}

[1] R. Siegel, D. Naishadham, and A. Jemal, "Cancer statistics, 2012," CA: A Cancer Journal for Clinicians, vol. 62, no. 1, pp. 10-29, 2012.

[2] P. Mehlen and A. Puisieux, "Metastasis: a question of life or death," Nature Reviews Cancer, vol. 6, no. 6, pp. 449-458, Jun. 2006.

[3] W. J. Allard, J. Matera, M. C. Miller, M. Repollet, M. C. Connelly, C. Rao, A. G. J. Tibbe, J. W. Uhr, and L. W. M. M. Terstappen, "Tumor Cells Circulate in the Peripheral Blood of All Major Carcinomas but not in Healthy Subjects or Patients With Nonmalignant Diseases," Clinical Cancer Research, vol. 10, no. 20, pp. 68976904, Oct. 2004.

[4] M. Cristofanilli, G. T. Budd, M. J. Ellis, A. Stopeck, J. Matera, M. C. Miller, J. M. Reuben, G. V. Doyle, W. J. Allard, L. W. M. M. Terstappen, and D. F. Hayes, "Circulating Tumor Cells, Disease Progression, and Survival in Metastatic Breast Cancer," New England Journal of Medicine, vol. 351, no. 8, pp. 781-791, 2004.

[5] S. Riethdorf and K. Pantel, "Advancing personalized cancer therapy by detection and characterization of circulating carcinoma cells," Annals of the New York Academy of Sciences, vol. 1210, no. 1, pp. 66-77, 2010.

[6] D. F. Hayes, M. Cristofanilli, G. T. Budd, M. J. Ellis, A. Stopeck, M. C. Miller, J. Matera, W. J. Allard, G. V. Doyle, and L. W. W. M. Terstappen, "Circulating Tumor Cells at Each Follow-up Time Point during Therapy of Metastatic Breast Cancer Patients Predict Progression-Free and Overall Survival," Clinical Cancer Research, vol. 12, no. 14, pp. 4218-4224, Jul. 2006. 
[7] S. Riethdorf, H. Fritsche, V. Müller, T. Rau, C. Schindlbeck, B. Rack, W. Janni, C. Coith, K. Beck, F. Jänicke, S. Jackson, T. Gornet, M. Cristofanilli, and K. Pantel, "Detection of Circulating Tumor Cells in Peripheral Blood of Patients with Metastatic Breast Cancer: A Validation Study of the CellSearch System," Clinical Cancer Research, vol. 13, no. 3, pp. 920-928, Feb. 2007.

[8] S. J. Cohen, C. J. A. Punt, N. Iannotti, B. H. Saidman, K. D. Sabbath, N. Y. Gabrail, J. Picus, M. Morse, E. Mitchell, M. C. Miller, G. V. Doyle, H. Tissing, L. W. M. M. Terstappen, and N. J. Meropol, "Relationship of Circulating Tumor Cells to Tumor Response, Progression-Free Survival, and Overall Survival in Patients With Metastatic Colorectal Cancer," Journal of Clinical Oncology, vol. 26, no. 19, pp. 3213-3221, Jul. 2008.

[9] G. T. Budd, M. Cristofanilli, M. J. Ellis, A. Stopeck, E. Borden, M. C. Miller, J. Matera, M. Repollet, G. V. Doyle, L. W. M. M. Terstappen, and D. F. Hayes, "Circulating Tumor Cells versus Imaging - Predicting Overall Survival in Metastatic Breast Cancer," Clinical Cancer Research, vol. 12, no. 21, pp. 6403-6409, Nov. 2006.

[10] M. Yu, S. Stott, M. Toner, S. Maheswaran, and D. A. Haber, "Circulating tumor cells: approaches to isolation and characterization," The Journal of Cell Biology, vol. 192, no. 3, pp. 373-382, Feb. 2011.

[11] A. Briggs and O. Kolosov, Acoustic microscopy. USA: Oxford University Press, 2009.

[12] K. T. Dussik, "On the possibility of using ultrasound waves as a diagnostic aid," $Z$ Neurol Psychiatr, vol. 174, pp. 153-168, 1942.

[13] R. S. C. Cobbold, Foundations of Biomedical Ultrasound, 1st ed. Oxford University Press, 2006.

[14] R. G. Maev, Acoustic Microscopy: Fundamentals and Applications. Weinheim, Germany: John Wiley \& Sons, 2008. 
[15] M. D. Sherar, M. B. Noss, and F. S. Foster, "Ultrasound backscatter microscopy images the internal structure of living tumour spheroids," Nature, vol. 330, no. 6147, pp. 493-495, 1987.

[16] F. L. Lizzi, M. Greenebaum, E. J. Feleppa, M. Elbaum, and D. J. Coleman, "Theoretical framework for spectrum analysis in ultrasonic tissue characterization," The Journal of the Acoustical Society of America, vol. 73, no. 4, pp. 1366-1373, 1983.

[17] F. L. Lizzi, M. Ostromogilsky, E. J. Feleppa, M. C. Rorke, and M. M. Yaremko, "Relationship of Ultrasonic Spectral Parameters to Features of Tissue Microstructure," IEEE Transactions on Ultrasonics, Ferroelectrics and Frequency Control, vol. 34, no. 3, pp. 319 -329, May 1987.

[18] F. L. Lizzi, M. Astor, E. J. Feleppa, M. Shao, and A. Kalisz, "Statistical framework for ultrasonic spectral parameter imaging," Ultrasound in Medicine \& Biology, vol. 23, no. 9, pp. 1371-1382, 1997.

[19] A. Nair, B. D. Kuban, E. M. Tuzcu, P. Schoenhagen, S. E. Nissen, and D. G. Vince, “Coronary Plaque Classification With Intravascular Ultrasound Radiofrequency Data Analysis," Circulation, vol. 106, no. 17, pp. 2200-2206, Oct. 2002.

[20] A. Nair, B. D. Kuban, N. Obuchowski, and D. G. Vince, "Assessing spectral algorithms to predict atherosclerotic plaque composition with normalized and raw intravascular ultrasound data," Ultrasound in Medicine \& Biology, vol. 27, no. 10, pp. 1319-1331, Oct. 2001.

[21] J. Mamou, M. L. Oelze, J. William D. O’Brien, and J. F. Zachary, "Identifying ultrasonic scattering sites from three-dimensional impedance maps," The Journal of the Acoustical Society of America, vol. 117, no. 1, pp. 413-423, 2005.

[22] R. Lavarello and M. Oelze, "Quantitative ultrasound estimates from populations of scatterers with continuous size distributions," IEEE Transactions on Ultrasonics, Ferroelectrics and Frequency Control, vol. 58, no. 4, pp. 744-53, Apr. 2011. 
[23] A. J. Dapore, M. R. King, J. Harter, S. Sarwate, M. L. Oelze, J. A. Zagzebski, M. N. Do, T. J. Hall, and W. D. O’Brien, "Analysis of Human Fibroadenomas Using ThreeDimensional Impedance Maps," IEEE Transactions on Medical Imaging, vol. 30, no. 6, pp. $1206-1213$, Jun. 2011.

[24] G. Czarnota, M. Kolios, H. Vaziri, and S. Benchimol, "Ultrasonic biomicroscopy of viable, dead and apoptotic cells," Ultrasound in Medicine \& Biology, vol. 23, no. 6, pp. 961-965, 1997.

[25] M. C. Kolios, G. J. Czarnota, M. Lee, J. W. Hunt, and M. D. Sherar, "Ultrasonic spectral parameter characterization of apoptosis," Ultrasound in Medicine \& Biology, vol. 28, no. 5, pp. 589-597, May 2002.

[26] B. Banihashemi, R. Vlad, B. Debeljevic, A. Giles, M. C. Kolios, and G. J. Czarnota, "Ultrasound imaging of apoptosis in tumor response: novel preclinical monitoring of photodynamic therapy effects," Cancer Research, vol. 68, no. 20, pp. 8590-6, Oct. 2008.

[27] A. Sadeghi-Naini, N. Papanicolau, O. Falou, J. Zubovits, R. Dent, S. Verma, M. Trudeau, J. F. Boileau, J. Spayne, S. Iradji, E. Sofroni, J. Lee, S. Lemon-Wong, M. Yaffe, M. C. Kolios, and G. J. Czarnota, "Quantitative ultrasound evaluation of tumor cell death response in locally advanced breast cancer patients receiving chemotherapy," Clinical Cancer Research, vol. 19, no. 8, pp. 2163-2174, Apr. 2013.

[28] M. L. Oelze, W. D. O'Brien, J. P. Blue, and J. F. Zachary, "Differentiation and characterization of rat mammary fibroadenomas and 4T1 mouse carcinomas using quantitative ultrasound imaging," IEEE Transactions on Medical Imaging, vol. 23, no. 6, pp. $764-771$, Jun. 2004.

[29] J. Mamou, A. Coron, M. Hata, J. Machi, E. Yanagihara, P. Laugier, and E. J. Feleppa, "Three-Dimensional High-Frequency Characterization of Cancerous Lymph Nodes," Ultrasound in Medicine \& Biology, vol. 36, no. 3, pp. 361-375, Mar. 2010.

[30] J. Mamou, A. Coron, M. L. Oelze, E. Saegusa-Beecroft, M. Hata, P. Lee, J. Machi, E. Yanagihara, P. Laugier, and E. J. Feleppa, "Three-Dimensional High-Frequency 
Backscatter and Envelope Quantification of Cancerous Human Lymph Nodes," Ultrasound in Medicine \& Biology, vol. 37, no. 3, pp. 345-357, Mar. 2011.

[31] E. Saegusa-Beecroft, J. Machi, J. Mamou, M. Hata, A. Coron, E. T. Yanagihara, T. Yamaguchi, M. L. Oelze, P. Laugier, and E. J. Feleppa, "Three-dimensional quantitative ultrasound for detecting lymph node metastases," in Journal of Surgical Research, 2011, vol. 183, pp. 258-269.

[32] E. Hysi, R. K. Saha, and M. C. Kolios, "On the use of photoacoustics to detect red blood cell aggregation," Biomedical Optics Express, vol. 3, no. 9, pp. 2326-2338, Sep. 2012.

[33] E. Hysi, R. K. Saha, and M. C. Kolios, "Photoacoustic ultrasound spectroscopy for assessing red blood cell aggregation and oxygenation," Journal of Biomedical Optics, vol. 17, no. 12, pp. 125006-125006, Dec. 2012.

[34] R. K. Saha, E. Franceschini, and G. Cloutier, "Assessment of accuracy of the structure-factor-size-estimator method in determining red blood cell aggregate size from ultrasound spectral backscatter coefficient," The Journal of the Acoustical Society of America, vol. 129, no. 4, pp. 2269-77, Apr. 2011.

[35] F. A. Duck, Physical Properties of Tissue: A Comprehensive Reference Book. San Diego: Academic Press, 1990.

[36] R. A. Lemons and C. F. Quate, "Acoustic microscope-scanning version," Applied Physics Letters, vol. 24, no. 4, p. 163, Feb. 1974.

[37] R. Lemons and C. Quate, “Acoustic microscopy: biomedical applications,” Science, vol. 188, no. 4191, pp. 905 -911, May 1975.

[38] R. N. Johnston, A. Atalar, J. Heiserman, V. Jipson, and C. F. Quate, "Acoustic microscopy: resolution of subcellular detail," Proceedings of the National Academy of Sciences, vol. 76, no. 7, pp. 3325 -3329, Jul. 1979.

[39] H. K. Wickramasinghe, "Contrast and imaging performance in the scanning acoustic microscope," Journal of Applied Physics, vol. 50, no. 2, p. 664, 1979. 
[40] J. A. Hildebrand, D. Rugar, R. N. Johnston, and C. F. Quate, "Acoustic microscopy of living cells," Proceedings of the National Academy of Sciences, vol. 78, no. 3, pp. 1656 -1660, Mar. 1981.

[41] J. A. Hildebrand and D. Rugar, "Measurement of cellular elastic properties by acoustic microscopy," Journal of Microscopy, vol. 134, no. 3, pp. 245-260, Jun. 1984.

[42] J. Litniewski and J. Bereiter-Hahn, "Measurements of cells in culture by scanning acoustic microscopy,” Journal of Microscopy, vol. 158, no. 1, pp. 95-107, Apr. 1990.

[43] H. Lüers, K. Hillmann, J. Litniewski, and J. Bereiter-Hahn, "Acoustic microscopy of cultured cells," Cell Biochemistry and Biophysics, vol. 18, no. 3, pp. 279-293, 1991.

[44] A. Atalar, C. F. Quate, and H. K. Wickramasinghe, "Phase imaging in reflection with the acoustic microscope," Applied Physics Letters, vol. 31, no. 12, pp. 791-793, Dec. 1977.

[45] T. Kundu, J. Bereiter-Hahn, and K. Hillmann, "Measuring elastic properties of cells by evaluation of scanning acoustic microscopy $\mathrm{V}(\mathrm{Z})$ values using simplex algorithm," Biophysical Journal, vol. 59, no. 6, pp. 1194-1207, Jun. 1991.

[46] T. Kundu, J. Bereiter-Hahn, and I. Karl, "Cell Property Determination from the Acoustic Microscope Generated Voltage Versus Frequency Curves," Biophysical Journal, vol. 78, no. 5, pp. 2270-2279, May 2000.

[47] G. A. D. Briggs, J. Wang, and R. Gundle, "Quantitative acoustic microscopy of individual living human cells," Journal of Microscopy, vol. 172, no. 1, pp. 3-12, 1993.

[48] C. M. Daft, G. A. Briggs, and W. D. O'Brien, "Frequency dependence of tissue attenuation measured by acoustic microscopy," The Journal of the Acoustical Society of America, vol. 85, no. 5, pp. 2194-201, May 1989.

[49] E. C. Weiss, P. Anastasiadis, G. Pilarczyk, R. M. Lemor, and P. V. Zinin, "Mechanical Properties of Single Cells by High-Frequency Time-Resolved Acoustic 
Microscopy," IEEE Transactions on Ultrasonics, Ferroelectrics and Frequency Control, vol. 54, no. 11, pp. 2257-2271, Nov. 2007.

[50] E. M. Strohm, G. J. Czarnota, and M. C. Kolios, “Acoustic microscopy of cells," in Quantitative ultrasound of soft tissue, Springer, 2013.

[51] A. Bell, "On the Production and Reproduction of Sound by Light," American Journal of Sciences, vol. XX, no. 118, pp. 305-324, Oct. 1880.

[52] G. Diebold, "Photoacoustic monopole radiation: waves from objects with symmetry in one, two and three dimensions," in Photo-acoustic Imaging and Spectroscopy, L. Wang, Ed. Taylor and Francis, 2009, pp. 3-17.

[53] P. M. Morse and K. U. Ingard, Theoretical Acoustics. McGraw-Hill Inc.,US, 1968.

[54] G. Diebold and P. Westervelt, "The photoacoustic effect generated by a spherical droplet in a fluid," The Journal of the Acoustical Society of America, vol. 84, p. 2245 , 1988.

[55] G. J. Diebold, M. I. Khan, and S. M. Park, "Photoacoustic 'Signatures' of Particulate Matter: Optical Production of Acoustic Monopole Radiation,” Science, vol. 250, no. 4977, pp. 101-104, Oct. 1990.

[56] G. J. Diebold, T. Sun, and M. I. Khan, "Photoacoustic monopole radiation in one, two, and three dimensions," Physical Review Letters, vol. 67, no. 24, pp. 3384-3387, 1991.

[57] M. Khan, T. Sun, and G. Diebold, "Photoacoustic waves generated by absorption of laser radiation in optically thin layers," The Journal of the Acoustical Society of America, vol. 93, no. 3, p. 1417, 1993.

[58] M. Khan, T. Sun, and G. Diebold, "Photoacoustic waves generated by absorption of laser radiation in optically thin cylinders," The Journal of the Acoustical Society of America, vol. 94, no. 2, p. 931, 1993.

[59] G. Paltauf and P. Dyer, "Photomechanical processes and effects in ablation," Chemical Reviews, vol. 103, no. 2, pp. 487-518, 2003. 
[60] K. Maslov, H. F. Zhang, S. Hu, and L. V. Wang, "Optical-resolution photoacoustic microscopy for in vivo imaging of single capillaries," Optics Letters, vol. 33, no. 9, pp. 929-31, May 2008.

[61] S. Hu and L. V. Wang, "Photoacoustic imaging and characterization of the microvasculature," Journal of Biomedical Optics, vol. 15, no. 1, pp. 011101-011101, Jan. 2010.

[62] R. G. M. Kolkman, P. J. Brands, W. Steenbergen, and T. G. van Leeuwen, "Real-time in vivo photoacoustic and ultrasound imaging," Journal of Biomedical Optics, vol. 13, no. 5, p. 050510, 2008.

[63] H. F. Zhang, K. Maslov, and L. V. Wang, "In vivo imaging of subcutaneous structures using functional photoacoustic microscopy," Nature Protocols, vol. 2, no. 4, pp. 797-804, Jan. 2007.

[64] L. V. Wang, "Multiscale photoacoustic microscopy and computed tomography," Nature Photonics, vol. 3, no. 9, pp. 503-509, 2009.

[65] L. V. Wang and S. Hu, "Photoacoustic Tomography: In Vivo Imaging from Organelles to Organs," Science, vol. 335, no. 6075, pp. 1458-1462, Mar. 2012.

[66] S. Mallidi, G. P. Luke, and S. Emelianov, "Photoacoustic imaging in cancer detection, diagnosis, and treatment guidance," Trends in Biotechnology, vol. 29, no. 5, pp. $213-$ 221, May 2011.

[67] C. Zhang, K. Maslov, and L. V. Wang, "Subwavelength-resolution label-free photoacoustic microscopy of optical absorption in vivo," Optics Letters, vol. 35, no. 19, pp. 3195-3197, Oct. 2010.

[68] G. Ku, K. Maslov, L. Li, and L. V. Wang, "Photoacoustic microscopy with 2-micron transverse resolution," Journal of Biomedical Optics, vol. 15, no. 2, p. 021302, 2010.

[69] S. Yang, F. Ye, and D. Xing, "Intracellular label-free gold nanorods imaging with photoacoustic microscopy," Optics Express, vol. 20, no. 9, pp. 10370-10375, Apr. 2012. 
[70] W. Bost, R. Lemor, and M. Fournelle, "Comparison of the optoacoustic signal generation efficiency of different nanoparticular contrast agents," Applied Optics, vol. 51, no. 33, pp. 8041-8046, Nov. 2012.

[71] T. Harrison, J. C. Ranasinghesagara, H. Lu, K. Mathewson, A. Walsh, and R. J. Zemp, "Combined photoacoustic and ultrasound biomicroscopy," Optics Express, vol. 17, no. 24, pp. 22041-22046, 2009.

[72] L. Wang, K. Maslov, J. Yao, B. Rao, and L. V. Wang, "Fast voice-coil scanning optical-resolution photoacoustic microscopy," Optics Letters, vol. 36, no. 2, pp. 139141, Jan. 2011.

[73] D.-K. Yao, K. Maslov, K. K. Shung, Q. Zhou, and L. V. Wang, "In vivo label-free photoacoustic microscopy of cell nuclei by excitation of DNA and RNA," Optics Letters, vol. 35, no. 24, pp. 4139-41, Dec. 2010.

[74] R. J. Zemp, L. Song, R. Bitton, K. K. Shung, and L. V. Wang, "Realtime photoacoustic microscopy in vivo with a 30-MHz ultrasound array transducer," Optics Express, vol. 16, no. 11, pp. 7915-28, May 2008.

[75] R. L. Shelton and B. E. Applegate, “Off-Axis Photoacoustic Microscopy," IEEE Transactions on Biomedical Engineering, vol. 57, no. 8, pp. 1835-1838, 2010.

[76] J.-T. Oh, M.-L. Li, H. F. Zhang, K. Maslov, G. Stoica, and L. V. Wang, "Threedimensional imaging of skin melanoma in vivo by dual-wavelength photoacoustic microscopy," Journal of Biomedical Optics, vol. 11, no. 3, pp. 034032-034032, May 2006.

[77] Y. Yuan, S. Yang, and D. Xing, "Optical-resolution photoacoustic microscopy based on two-dimensional scanning galvanometer," Applied Physics Letters, vol. 100, no. 2, pp. 023702-023702-3, Jan. 2012.

[78] Z. Xie, S. Jiao, H. F. Zhang, and C. A. Puliafito, "Laser-scanning optical-resolution photoacoustic microscopy," Optics Letters, vol. 34, no. 12, pp. 1771-1773, Jun. 2009. 
[79] E. Zhang, J. Laufer, and P. Beard, "Backward-mode multiwavelength photoacoustic scanner using a planar Fabry-Perot polymer film ultrasound sensor for high-resolution three-dimensional imaging of biological tissues," Applied Optics, vol. 47, no. 4, pp. 561-577, Feb. 2008.

[80] E. M. Strohm, E. S. L. Berndl, and M. C. Kolios, "High frequency label-free photoacoustic microscopy of single cells," Photoacoustics, vol. 1, no. 3-4, pp. 49-53, Dec. 2013.

[81] E. M. Strohm, E. Hysi, and M. C. Kolios, "Photoacoustic measurements of single red blood cells," in IEEE International Ultrasonics Symposium, Dresden, Germany, 2012.

[82] G. Bao and S. Suresh, "Cell and molecular mechanics of biological materials," Nature Materials, vol. 2, no. 11, pp. 715-725, Nov. 2003.

[83] S. Suresh, "Biomechanics and biophysics of cancer cells," Acta Materialia, vol. 55, no. 12, pp. 3989-4014, 2007.

[84] Y. Park, C. A. Best, T. Auth, N. S. Gov, S. A. Safran, G. Popescu, S. Suresh, and M. S. Feld, "Metabolic remodeling of the human red blood cell membrane," Proceedings of the National Academy of Sciences, vol. 107, no. 4, pp. 1289-1294, Jan. 2010.

[85] Y. Park, C. A. Best, K. Badizadegan, R. R. Dasari, M. S. Feld, T. Kuriabova, M. L. Henle, A. J. Levine, and G. Popescu, "Measurement of red blood cell mechanics during morphological changes," Proceedings of the National Academy of Sciences, vol. 107, no. 15, pp. 6731-6736, Apr. 2010.

[86] G. J. Czarnota, M. C. Kolios, J. Abraham, M. Portnoy, F. P. Ottensmeyer, J. W. Hunt, and M. D. Sherar, "Ultrasound imaging of apoptosis: high-resolution non-invasive monitoring of programmed cell death in vitro, in situ and in vivo," British Journal of Cancer, vol. 81, no. 3, pp. 520-527, Oct. 1999.

[87] A. S. Tunis, G. Czarnota, A. Giles, M. D. Sherar, J. W. Hunt, and M. C. Kolios, "Monitoring structural changes in cells with high-frequency ultrasound signal statistics," Ultrasound in Medicine \& Biology, vol. 31, no. 8, pp. 1041-9, 2005. 
[88] M. C. Kolios and G. J. Czarnota, "Potential use of ultrasound for the detection of cell changes in cancer treatment," Future Oncology, vol. 5, no. 10, pp. 1527-1532, 2009.

[89] G. J. Czarnota and M. C. Kolios, "Ultrasound detection of cell death," Imaging in Medicine, vol. 2, no. 1, pp. 17-28, Feb. 2010.

[90] S. Yamada, D. Wirtz, and S. C. Kuo, "Mechanics of Living Cells Measured by Laser Tracking Microrheology," Biophysical Journal, vol. 78, no. 4, pp. 1736-1747, Apr. 2000.

[91] D. Wirtz, "Particle-tracking microrheology of living cells: principles and applications," Annual Review of Biophysics, vol. 38, pp. 301-26, Jan. 2009.

[92] Y. Tseng, T. P. Kole, and D. Wirtz, "Micromechanical Mapping of Live Cells by Multiple-Particle-Tracking Microrheology," Biophysical Journal, vol. 83, no. 6, pp. 3162-3176, Dec. 2002.

[93] Y. Li, J. Schnekenburger, and M. H. G. Duits, "Intracellular particle tracking as a tool for tumor cell characterization," Journal of Biomedical Optics, vol. 14, no. 6, p. 064005, 2009.

[94] W. R. Trickey, F. P. T. Baaijens, T. a Laursen, L. G. Alexopoulos, and F. Guilak, "Determination of the Poisson's ratio of the cell: recovery properties of chondrocytes after release from complete micropipette aspiration," Journal of Biomechanics, vol. 39, no. 1, pp. 78-87, Jan. 2006.

[95] R. M. Hochmuth, "Micropipette aspiration of living cells," Journal of Biomechanics, vol. 33, no. 1, pp. 15-22, Jan. 2000.

[96] M. Dao, C. T. Lim, and S. Suresh, "Mechanics of the human red blood cell deformed by optical tweezers," Journal of the Mechanics and Physics of Solids, vol. 51, no. 1112, pp. 2259-2280, Nov. 2003.

[97] S. Mohanty, K. Mohanty, and P. Gupta, "Dynamics of Interaction of RBC with optical tweezers,” Optics Express, vol. 13, no. 12, pp. 4745-4751, Jun. 2005. 
[98] E. A-Hassan, W. F. Heinz, M. D. Antonik, N. P. D’Costa, S. Nageswaran, C. a Schoenenberger, and J. H. Hoh, "Relative microelastic mapping of living cells by atomic force microscopy," Biophysical Journal, vol. 74, no. 3, pp. 1564-78, Mar. 1998.

[99] H. Haga, S. Sasaki, K. Kawabata, E. Ito, T. Ushiki, and T. Sambongi, "Elasticity mapping of living fibroblasts by AFM and immunofluorescence observation of the cytoskeleton," Ultramicroscopy, vol. 82, no. 1-4, pp. 253-258, Feb. 2000.

[100] R. Matzke, K. Jacobson, and M. Radmacher, "Direct, high-resolution measurement of furrow stiffening during division of adherent cells," Nature Cell Biology, vol. 3, no. 6, pp. 607-10, Jun. 2001.

[101] K. D. Costa, "Single-cell elastography: probing for disease with the atomic force microscope," Disease Markers, vol. 19, no. 2-3, pp. 139-154, 20042003.

[102] S. E. Cross, Y.-S. Jin, J. Tondre, R. Wong, J. Rao, and J. K. Gimzewski, “AFM-based analysis of human metastatic cancer cells," Nanotechnology, vol. 19, no. 38, p. 384003, 2008.

[103] Q. S. Li, G. Y. H. Lee, C. N. Ong, and C. T. Lim, “AFM indentation study of breast cancer cells," Biochemical and Biophysical Research Communications, vol. 374, no. 4, pp. 609-13, Oct. 2008.

[104] M. Lekka and P. Laidler, "Applicability of AFM in cancer detection," Nature Nanotechnology, vol. 4, no. 2, pp. 72-73, Feb. 2009.

[105] Q. Guo, Y. Xia, M. Sandig, and J. Yang, "Characterization of cell elasticity correlated with cell morphology by atomic force microscope," Journal of Biomechanics, vol. 45, no. 2, pp. 304-309, Jan. 2012.

[106] E. M. Strohm, G. J. Czarnota, and M. C. Kolios, "Quantitative measurements of apoptotic cell properties using acoustic microscopy," IEEE Transactions on Ultrasonics, Ferroelectrics and Frequency Control, vol. 57, no. 10, pp. 2293-2304, Oct. 2010. 
[107] M. Martin, K. Mueller, F. Wottawah, S. Schinkinger, B. Lincoln, M. Romeyke, and J. A. Käs, "Feeling with light for cancer," in Proceedings of SPIE, 2006, p. 60800P60800P.

[108] L. R. Taggart, R. E. Baddour, A. Giles, G. J. Czarnota, and M. C. Kolios, "Ultrasonic characterization of whole cells and isolated nuclei," Ultrasound in Medicine \& Biology, vol. 33, no. 3, pp. 389-401, 2007.

[109] B. D. Chithrani, A. A. Ghazani, and W. C. W. Chan, "Determining the Size and Shape Dependence of Gold Nanoparticle Uptake into Mammalian Cells," Nano Letters, vol. 6, no. 4, pp. 662-668, 2006.

[110] M. C. Jamur and C. Oliver, "Permeabilization of Cell Membranes," in Immunocytochemical Methods and Protocols, vol. 588, C. Oliver and M. C. Jamur, Eds. Totowa, NJ: Humana Press, 2010, pp. 63-66.

[111] A. L. van de Ven, K. Adler-Storthz, and R. Richards-Kortum, "Delivery of optical contrast agents using Triton-X100, part 1: reversible permeabilization of live cells for intracellular labeling," Journal of Biomedical Optics, vol. 14, no. 2, pp. 021012021012 , Mar. 2009.

[112] A. L. van de Ven, K. Adler-Storthz, and R. Richards-Kortum, "Delivery of optical contrast agents using Triton-X100, part 2: enhanced mucosal permeation for the detection of cancer biomarkers," Journal of Biomedical Optics, vol. 14, no. 2, pp. 021013-021013, Mar. 2009.

[113] E. I. Galanzha and V. P. Zharov, "Photoacoustic flow cytometry," Methods, vol. 57, no. 3, pp. 280-296, Jul. 2012.

[114] P. Beard, "Biomedical photoacoustic imaging," Interface Focus, vol. 1, no. 4, pp. 602-631, Aug. 2011.

[115] M. A. Proskurnin, T. V. Zhidkova, D. S. Volkov, M. Sarimollaoglu, E. I. Galanzha, D. Mock, D. A. Nedosekin, and V. P. Zharov, "In vivo multispectral photoacoustic and photothermal flow cytometry with multicolor dyes: A potential for real-time 
assessment of circulation, dye-cell interaction, and blood volume," Cytometry Part A, vol. 79A, no. 10, pp. 834-847, Oct. 2011.

[116] E. I. Galanzha, E. V. Shashkov, V. V. Tuchin, and V. P. Zharov, "In vivo multispectral, multiparameter, photoacoustic lymph flow cytometry with natural cell focusing, label-free detection and multicolor nanoparticle probes," Cytometry Part A, vol. 73A, no. 10, pp. 884-894, Oct. 2008.

[117] S. Mallidi, T. Larson, J. Tam, P. P. Joshi, A. Karpiouk, K. Sokolov, and S. Emelianov, "Multiwavelength Photoacoustic Imaging and Plasmon Resonance Coupling of Gold Nanoparticles for Selective Detection of Cancer," Nano Letters, vol. 9, no. 8, pp. 2825-2831, Aug. 2009.

[118] R. E. Baddour, M. D. Sherar, J. W. Hunt, G. J. Czarnota, and M. C. Kolios, "Highfrequency ultrasound scattering from microspheres and single cells," Journal of the Acoustical Society of America, vol. 117, no. 2, p. 934, 2005.

[119] T. Sun and G. Diebold, "Generation of ultrasonic waves from a layered photoacoustic source," Nature Letters, vol. 355, p. 806, 1992.

[120] V. C. Anderson, "Sound Scattering from a Fluid Sphere," The Journal of the Acoustical Society of America, vol. 22, no. 4, p. 426, 1950.

[121] J. Faran, "Sound scattering by solid cylinders and spheres," The Journal of the Acoustical Society of America, vol. 23, no. 4, pp. 405-418, 1951.

[122] R. Hickling, "Analysis of echoes from a solid elastic sphere in water," Journal of the Acoustical Society of America, vol. 34, no. 10, pp. 1582-1592, 1962.

[123] O. Falou, R. E. Baddour, G. Nathanael, G. J. Czarnota, J. C. Kumaradas, and M. C. Kolios, "A study of high frequency ultrasound scattering from non-nucleated biological specimens," The Journal of the Acoustical Society of America, vol. 124, no. 5, pp. EL278-83, 2008.

[124] O. Falou, M. Rui, A. El-Kaffas, J. C. Kumaradas, and M. C. Kolios, "A novel technique for measuring ultrasound backscatter from single micron-sized objects," in IEEE Ultrasonics Symposium, 2009, pp. 291-293. 
[125] O. Falou, M. Rui, A. El Kaffas, J. C. Kumaradas, and M. C. Kolios, "The measurement of ultrasound scattering from individual micron-sized objects and its application in single cell scattering," The Journal of the Acoustical Society of America, vol. 128, no. 2, p. 894, 2010.

[126] O. Falou, A. J. Sojahrood, J. C. Kumaradas, and M. C. Kolios, "Surface modes and acoustic scattering of microspheres and ultrasound contrast agents," The Journal of the Acoustical Society of America, vol. 132, no. 3, pp. 1820-1829, 2012.

[127] W. Bost, F. Stracke, E. C. Weiss, S. Narasimhan, M. C. Kolios, and R. Lemor, "High frequency optoacoustic microscopy," in Annual International Conference of the IEEE Engineering in Medicine and Biology Society, 2009, vol. 2009, pp. 5883-6.

[128] M. Rui, S. Narashimhan, W. Bost, F. Stracke, E. Weiss, R. Lemor, and M. C. Kolios, "Gigahertz optoacoustic imaging for cellular imaging," in Proceedings of SPIE, 2010, vol. 7564, pp. 756411-756411-6.

[129] M. Rui, W. Bost, E. C. Weiss, R. Lemor, and M. C. Kolios, "Photoacoustic Microscopy and Spectroscopy of Individual Red Blood Cells," International Journal of Radiation Oncology, Biology, Physics, pp. 3-5, 2010.

[130] O. Falou, J. Kumaradas, and M. Kolios, "A study of femlab for modeling high frequency ultrasound scattering by spherical objects," in FEMLAB User Conference, 2005.

[131] O. Falou, J. Kumaradas, and M. Kolios, "Modeling acoustic wave scattering from cells and microbubbles," in Proceedings of Comsol, 2006.

[132] M. C. Kolios, O. Falou, and J. C. Kumaradas, "Finite Element Modeling of Ultrasound Scattering by Spherical Objects and Cells," in IEEE Ultrasonics Symposium, 2006, pp. 2072-2075.

[133] O. Falou, J. Kumaradas, and M. Kolios, "Finite-element Modeling of Elastic Surface Modes and Scattering from Spherical Objects," in Proceedings of Comsol, 2007. 
[134] K. Kim, Z. Wang, and S.-H. Ha, "Photoacoustic design parameter optimization for deep tissue imaging by numerical simulation," in Proceedings of SPIE, pp. 822346822346, Feb. 2012.

[135] Z. Wang, S. Ha, and K. Kim, "Evaluation of finite-element-based simulation model of photoacoustics in biological tissues," in Proceedings of SPIE, 2012, vol. 8320, p. 83201L-83201L-9.

[136] Y.-L. Sheu and P.-C. Li, "Simulations of thermally induced photoacoustic wave propagation using a pseudospectral time-domain method," IEEE Transactions on Ultrasonics, Ferroelectrics and Frequency Control, vol. 56, no. 5, pp. 1104-12, May 2009.

[137] B. T. Cox and P. C. Beard, "Fast calculation of pulsed photoacoustic fields in fluids using k-space methods," The Journal of the Acoustical Society of America, vol. 117, no. 6, p. 3616, 2005.

[138] H. F. Zhang, K. Maslov, G. Stoica, and L. V. Wang, "Functional photoacoustic microscopy for high-resolution and noninvasive in vivo imaging," Nature Biotechnology, vol. 24, no. 7, pp. 848-851, 2006.

[139] X. Wang, Y. Pang, G. Ku, X. Xie, G. Stoica, and L. V. Wang, "Noninvasive laserinduced photoacoustic tomography for structural and functional in vivo imaging of the brain," Nature Biotechnology, vol. 21, no. 7, pp. 803-806, 2003.

[140] R. G. M. Kolkman, E. Hondebrink, W. Steenbergen, and F. F. M. de Mul, "In vivo photoacoustic imaging of blood vessels using an extreme-narrow aperture sensor," IEEE Journal of Selected Topics in Quantum Electronics, vol. 9, no. 2, pp. 343-346, Mar. 2003.

[141] J.-W. Kim, E. I. Galanzha, E. V. Shashkov, H.-M. Moon, and V. P. Zharov, "Golden carbon nanotubes as multimodal photoacoustic and photothermal high-contrast molecular agents," Nature Nanotechnology, vol. 4, no. 10, pp. 688-694, 2009.

[142] S. Manohar, S. E. Vaartjes, J. C. G. van Hespen, J. M. Klaase, F. M. van den Engh, W. Steenbergen, and T. G. van Leeuwen, "Initial results of in vivo non-invasive 
cancer imaging in the human breast using near-infrared photoacoustics," Optics Express, vol. 15, no. 19, pp. 12277-12285, Sep. 2007.

[143] A. Agarwal, S. W. Huang, M. O’Donnell, K. C. Day, M. Day, N. Kotov, and S. Ashkenazi, "Targeted gold nanorod contrast agent for prostate cancer detection by photoacoustic imaging," Journal of Applied Physics, vol. 102, no. 6, pp. 064701064701-4, 2007.

[144] E. M. Strohm, M. Rui, I. Gorelikov, N. Matsuura, and M. Kolios, "Vaporization of perfluorocarbon droplets using optical irradiation," Biomedical Optics Express, vol. 2, no. 6, pp. 1432-1442, 2011.

[145] K. Wilson, K. Homan, and S. Emelianov, "Biomedical photoacoustics beyond thermal expansion using triggered nanodroplet vaporization for contrast-enhanced imaging," Nature Communications, vol. 3, p. 618, Jan. 2012.

[146] P. S. Sheeran and P. A. Dayton, "Phase-change contrast agents for imaging and therapy," Current Pharmaceutical Design, vol. 18, no. 15, pp. 2152-2165, May 2012.

[147] N. Rapoport, K.-H. Nam, R. Gupta, Z. Gao, P. Mohan, A. Payne, N. Todd, X. Liu, T. Kim, J. Shea, C. Scaife, D. L. Parker, E.-K. Jeong, and A. M. Kennedy, "Ultrasoundmediated tumor imaging and nanotherapy using drug loaded, block copolymer stabilized perfluorocarbon nanoemulsions," Journal of Controlled Release, vol. 153, no. 1, pp. 4-15, Jul. 2011.

[148] E. M. Strohm, M. Rui, M. C. Kolios, I. Gorelikov, and N. Matsuura, "Optical droplet vaporization (ODV): Photoacoustic characterization of perfluorocarbon droplets," in IEEE International Ultrasonics Symposium, 2010, pp. 495-498.

[149] B. E. Treeby and B. T. Cox, "k-Wave: MATLAB toolbox for the simulation and reconstruction of photoacoustic wave fields," Journal of Biomedical Optics, vol. 15, no. 2, pp. 021314-021314-12, Mar. 2010.

[150] J. A. Jensen and N. B. Svendsen, "Calculation of pressure fields from arbitrarily shaped, apodized, and excited ultrasound transducers," IEEE Transactions on 
Ultrasonics, Ferroelectrics and Frequency Control, vol. 39, no. 2, pp. 262 -267, Mar. 1992.

[151] J. A. Jensen, "FIELD: A Program for Simulating Ultrasound Systems," in 10th Nordicbaltic Conference on Biomedical Imaging, 1996, vol. 4, pp. 351-353.

[152] B. Xu, "Numerical simulation of laser-generated ultrasound by the finite element method," Journal of Applied Physics, vol. 95, no. 4, p. 2116, 2004.

[153] B. Xu, Z. Shen, J. Wang, X. Ni, J. Guan, and J. Lu, "Thermoelastic finite element modeling of laser generation ultrasound," Journal of Applied Physics, vol. 99, no. 3, p. 033508, 2006.

[154] Z. Yuan and H. Jiang, "Three-dimensional finite-element-based photoacoustic tomography: Reconstruction algorithm and simulations," Medical Physics, vol. 34, no. 2, p. 538, 2007.

[155] L. Yao and H. Jiang, "Finite-element-based photoacoustic tomography in time domain," Journal of Optics A: Pure and Applied Optics, vol. 11, no. 8, p. 085301, Aug. 2009.

[156] Z. Wang, S. Ha, and K. Kim, "Evaluation of finite-element-based simulation model of photoacoustics in biological tissues," in Proceedings of SPIE, 2012, vol. 8320, p. 83201L-83201L-9.

[157] M. Kasai, S. Fukushima, Y. Gohshi, T. Sawada, M. Ishioka, and M. Kaihara, "A basic analysis of pulsed photoacoustic signals using the finite elements method," Journal of Applied Physics, vol. 64, no. 3, p. 972, 1988.

[158] J. F. Lovell, C. S. Jin, E. Huynh, H. Jin, C. Kim, J. L. Rubinstein, W. C. W. Chan, W. Cao, L. V. Wang, and G. Zheng, "Porphysome nanovesicles generated by porphyrin bilayers for use as multimodal biophotonic contrast agents," Nature Materials, vol. 10, no. 4, pp. 324-332, Mar. 2011.

[159] E. Huynh, J. F. Lovell, B. Helfield, M. Jeon, C. Kim, D. E. Goertz, B. C. Wilson, and G. Zheng, "Porphyrin Shell Microbubbles with Intrinsic Ultrasound and 
Photoacoustic Properties," Journal of the American Chemical Society, vol. 134, no. 40, pp. 16464-16467, 2012.

[160] M. A. Hines and G. D. Scholes, "Colloidal PbS Nanocrystals with Size-Tunable Near-Infrared Emission: Observation of Post-Synthesis Self-Narrowing of the Particle Size Distribution," Advanced Materials, vol. 15, no. 21, pp. 1844-1849, Nov. 2003.

[161] I. Gorelikov and N. Matsuura, "Single-step coating of mesoporous silica on cetyltrimethyl ammonium bromide-capped nanoparticles," Nano Letters, vol. 8, no. 1, pp. 369-73, Jan. 2008.

[162] D. K. Yi, S. T. Selvan, S. S. Lee, G. C. Papaefthymiou, D. Kundaliya, and J. Y. Ying, "Silica-coated nanocomposites of magnetic nanoparticles and quantum dots," Journal of the American Chemical Society, vol. 127, no. 14, pp. 4990-4991, Apr. 2005.

[163] N. Matsuura, I. Gorelikov, R. Williams, K. Wan, S. Zhu, J. Booth, P. Burns, K. Hynynen, and J. A. Rowlands, "Nanoparticle-Tagged Perfluorocarbon Droplets for Medical Imaging," in Materials Research Society Symposium Proceedings, 2009, vol. 1140.

[164] K. Zell, J. I. Sperl, M. W. Vogel, R. Niessner, and C. Haisch, “Acoustical properties of selected tissue phantom materials for ultrasound imaging," Physics in Medicine \& Biology, vol. 52, no. 20, pp. N475-84, Oct. 2007.

[165] O. D. Kripfgans, J. B. Fowlkes, D. L. Miller, O. P. Eldevik, and P. L. Carson, "Acoustic droplet vaporization for therapeutic and diagnostic applications," Ultrasound in Medicine \& Biology, vol. 26, no. 7, pp. 1177-1189, 2000.

[166] F. H. Jongsma, "Liquids with a very low ultrasound propagation velocity," Ultrasonics, vol. 17, no. 5, p. 233, Sep. 1979.

[167] K. L. Narayana and K. M. Swamy, "Ultrasonic velocity in and adiabatic compressibility for some fluorocarbon liquid mixtures," Journal of Chemical \& Engineering Data, vol. 34, no. 1, pp. 19-21, Jan. 1989. 
[168] J. N. Marsh, C. S. Hall, S. A. Wickline, and G. M. Lanza, "Temperature dependence of acoustic impedance for specific fluorocarbon liquids," Journal of the Acoustical Society of America, vol. 112, no. 6, pp. 2858-2862, 2002.

[169] E. M. Strohm and M. C. Kolios, "Sound velocity and attenuation measurements of perfluorocarbon liquids using photoacoustic methods," in IEEE International Ultrasonics Symposium, Orlando, FL, USA, 2011, pp. 2368-2371.

[170] Y. Sun, Chengcheng Niu, Yanjie Wang, Eric M. Strohm, Haitao Ran, Yuanyi Zheng, Zhigang Wang, and Michael C. Kolios, "Vaporization, Photoacoustic and Acoustic characterization of PLGA/PFH particles loaded with optically absorbing materials," in IEEE International Ultrasonics Symposium, Prague, Czech Republic, 2013.

[171] Y. Kohl, C. Kaiser, W. Bost, F. Stracke, M. Fournelle, C. Wischke, H. Thielecke, A. Lendlein, K. Kratz, and R. Lemor, "Preparation and biological evaluation of multifunctional PLGA-nanoparticles designed for photoacoustic imaging," Nanomedicine: Nanotechnology, Biology and Medicine, vol. 7, no. 2, pp. 228-237, Apr. 2011.

[172] D. V. Volodkin, A. G. Skirtach, and H. Möhwald, "Near-IR Remote Release from Assemblies of Liposomes and Nanoparticles," Angewandte Chemie International Edition, vol. 48, no. 10, pp. 1807-1809, 2009.

[173] D. T. Deihl, “N Waves’ from Bursting Balloons,” American Journal of Physics, vol. 36, no. 5, p. 441, 1968.

[174] E. M. Strohm, Elizabeth S.L. Berndl, and M. C. Kolios, "Probing red blood cell morphology using high frequency photoacoustics," Biophysical Journal, vol. 105, no. 1, pp. 59-67, Jul. 2013.

[175] E. M. Strohm, I. Gorelikov, N. Matsuura, and M. C. Kolios, "Acoustic and photoacoustic characterization of micron-sized perfluorocarbon emulsions," Journal of Biomedical Optics, vol. 17, no. 9, pp. 096016-1-9, Sep. 2012.

[176] C. S. Cohn and M. M. Cushing, "Oxygen therapeutics: perfluorocarbons and blood substitute safety," Critical Care Clinics, vol. 25, no. 2, pp. 399-414, Apr. 2009. 
[177] J. P. Fast and S. Mecozzi, "Nanoemulsions for Intravenous Drug Delivery," in Nanotechnology in Drug Delivery, M. M. Villiers, P. Aramwit, and G. S. Kwon, Eds. New York, NY: Springer New York, 2009, pp. 461-489.

[178] R. E. Apfel, "Activatable infusable dispersions containing drops of a superheated liquid,", Patent 584027624-Nov-1998.

[179] T. Giesecke and K. Hynynen, "Ultrasound-mediated cavitation thresholds of liquid perfluorocarbon droplets in vitro," Ultrasound in Medicine \& Biology, vol. 29, no. 9, pp. 1359-1365, 2003.

[180] P. S. Sheeran, V. P. Wong, S. Luois, R. J. McFarland, W. D. Ross, S. Feingold, T. O. Matsunaga, and P. A. Dayton, "Decafluorobutane as a Phase-Change Contrast Agent for Low-Energy Extravascular Ultrasonic Imaging," Ultrasound in Medicine \& Biology, vol. 37, no. 9, pp. 1518-1530, Sep. 2011.

[181] N. Reznik, R. Williams, and P. N. Burns, "Investigation of Vaporized Submicron Perfluorocarbon Droplets as an Ultrasound Contrast Agent," Ultrasound in Medicine \& Biology, vol. 37, no. 8, pp. 1271-1279, Aug. 2011.

[182] O. D. Kripfgans, J. B. Fowlkes, M. Woydt, O. P. Eldevik, and P. L. Carson, "In vivo droplet vaporization for occlusion therapy and phase aberration correction," IEEE Transactions on Ultrasonics, Ferroelectrics and Frequency Control, vol. 49, no. 6, pp. 726-738, 2002.

[183] M. Zhang, M. L. Fabiilli, K. J. Haworth, F. Padilla, S. D. Swanson, O. D. Kripfgans, P. L. Carson, and J. B. Fowlkes, “Acoustic Droplet Vaporization for Enhancement of Thermal Ablation by High Intensity Focused Ultrasound," Academic Radiology, vol. 18, no. 9, pp. 1123-1132, Sep. 2011.

[184] M. L. Fabiilli, K. J. Haworth, I. E. Sebastian, O. D. Kripfgans, P. L. Carson, and J. B. Fowlkes, "Delivery of chlorambucil using an acoustically-triggered perfluoropentane emulsion," Ultrasound in Medicine \& Biology, vol. 36, no. 8, pp. 1364-75, 2010. 
[185] K. Wilson, K. Homan, and S. Emelianov, "Photoacoustic and ultrasound imaging contrast enhancement using a dual contrast agent," in Proceedings of SPIE, 2010, vol. 7564, p. 75642P.

[186] W. J. Akers, C. Kim, M. Berezin, K. Guo, R. Fuhrhop, G. M. Lanza, G. M. Fischer, E. Daltrozzo, A. Zumbusch, X. Cai, L. V. Wang, and S. Achilefu, "Noninvasive Photoacoustic and Fluorescence Sentinel Lymph Node Identification using DyeLoaded Perfluorocarbon Nanoparticles," ACS Nano, vol. 5, no. 1, pp. 173-182, 2011.

[187] P. K. Jain, I. H. El-Sayed, and M. A. El-Sayed, "Au nanoparticles target cancer," Nano Today, vol. 2, no. 1, pp. 18-29, Feb. 2007.

[188] A. D. L. Zerda, C. Zavaleta, S. Keren, S. Vaithilingam, S. Bodapati, Z. Liu, J. Levi, B. R. Smith, T.-J. Ma, O. Oralkan, Z. Cheng, X. Chen, H. Dai, B. T. Khuri-Yakub, and S. S. Gambhir, "Carbon nanotubes as photoacoustic molecular imaging agents in living mice," Nature Nanotechnology, vol. 3, no. 9, pp. 557-562, 2008.

[189] H. Maeda, J. Wu, T. Sawa, Y. Matsumura, and K. Hori, "Tumor vascular permeability and the EPR effect in macromolecular therapeutics: a review," Journal of Controlled Release, vol. 65, no. 1-2, pp. 271-284, 2000.

[190] Y. Chen, W. Frey, S. Aglyamov, and S. Emelianov, "Environment-Dependent Generation of Photoacoustic Waves from Plasmonic Nanoparticles," Small, vol. 8, no. 1, pp. 47-52, Jan. 2012.

[191] M. M. Kaneda, S. Caruthers, G. M. Lanza, and S. A. Wickline, "Perfluorocarbon Nanoemulsions for Quantitative Molecular Imaging and Targeted Therapeutics," Annals of Biomedical Engineering, vol. 37, pp. 1922-1933, Jan. 2009.

[192] R. S. C. Cobbold, N. V. Sushilov, and A. C. Weathermon, "Transient propagation in media with classical or power-law loss," The Journal of the Acoustical Society of America, vol. 116, no. 6, pp. 3294-3303, Dec. 2004.

[193] M. O’Donnell, "Kramers-Kronig relationship between ultrasonic attenuation and phase velocity," The Journal of the Acoustical Society of America, vol. 69, no. 3, p. 696, 1981. 
[194] T. L. Szabo, "Causal theories and data for acoustic attenuation obeying a frequency power law," The Journal of the Acoustical Society of America, vol. 97, no. 1, p. 14, 1995.

[195] N. G. Bastús, J. Comenge, and V. Puntes, "Kinetically Controlled Seeded Growth Synthesis of Citrate-Stabilized Gold Nanoparticles of up to $200 \mathrm{~nm}$ : Size Focusing versus Ostwald Ripening," Langmuir, vol. 27, no. 17, pp. 11098-11105, 2011.

[196] L. M. Liz-Marzán, M. Giersig, and P. Mulvaney, "Synthesis of Nanosized Gold-Silica Core-Shell Particles,” Langmuir, vol. 12, no. 18, pp. 4329-4335, 1996.

[197] I. Gorelikov, A. L. Martin, M. Seo, and N. Matsuura, "Silica-Coated Quantum Dots for Optical Evaluation of Perfluorocarbon Droplet Interactions with Cells," Langmuir, vol. 27, no. 24, pp. 15024-15033, 2011.

[198] A. Martin, M. Seo, R. Williams, G. Belayneh, F. Foster, and N. Matsuura, "Intracellular growth of nanoscale perfluorocarbon droplets for enhanced ultrasoundinduced phase-change conversion," Ultrasound in Medicine \& Biology, 2012.

[199] M. G. Freire, A. M. A. Dias, M. A. Z. Coelho, J. A. P. Coutinho, and I. M. Marrucho, "Aging mechanisms of perfluorocarbon emulsions using image analysis," Journal of Colloid and Interface Science, vol. 286, no. 1, pp. 224-232, Jun. 2005.

[200] B. G. Watters, "The (Sound of a Bursting) Red Balloon," Sound: Its Uses and Control, vol. 2, no. 2, p. 8, 1963.

[201] R. R. Letfullin, C. Joenathan, T. F. George, and V. P. Zharov, "Laser-induced explosion of gold nanoparticles: potential role for nanophotothermolysis of cancer," Nanomedicine, vol. 1, no. 4, pp. 473-480, 2006.

[202] G. D. Scott and D. M. Kilgour, "The density of random close packing of spheres," Journal of Physics D: Applied Physics, vol. 2, no. 6, pp. 863-866, Jun. 1969.

[203] C. S. Hall, G. M. Lanza, J. H. Rose, R. J. Kaufmann, R. W. Fuhrhop, S. H. Handley, K. R. Waters, J. G. Miller, and S. A. Wickline, "Experimental determination of phase velocity of perfluorocarbons: Applications to targeted contrast agents," IEEE 
Transactions on Ultrasonics, Ferroelectrics and Frequency Control, vol. 47, no. 1, pp. 75-84, Jan. 2000.

[204] E. M. Strohm, M. Rui, I. Gorelikov, N. Matsuura, and M. Kolios, "Optical Droplet Vaporization of Micron-sized Perfluorocarbon Droplets and their Photoacoustic Detection," in Proceedings of SPIE, 2011, p. 78993H.

[205] E. Strohm, I. Gorelikov, N. Matsuura, and M. Kolios, "Photoacoustic spectral characterization of perfluorocarbon droplets," in Proceedings of SPIE, 2012, vol. 8223, p. 82232F.

[206] M. M. Wintrobe, J. P. Greer, and G. R. Lee, Wintrobe's Clinical Hematology. Philadelphia: Wolters Kluwer/Lippincott Williams \& Wilkins, 2009.

[207] S. Chien, "Red cell deformability and its relevance to blood flow," Annual Review of Physiology, vol. 49, pp. 177-192, 1987.

[208] Y. C. Fung, Biomechanics: Mechanical Properties of Living Tissues, Second Edition, 2nd ed. New York, NY: Springer, 1993.

[209] Hematology: Clinical Principles and Applications. Elsevier Health Sciences, 2007.

[210] H. M. Shapiro, Practical Flow Cytometry. John Wiley \& Sons, 2003.

[211] P. B. Canham and A. C. Burton, "Distribution of Size and Shape in Populations of Normal Human Red Cells," Circulation Research, vol. 22, no. 3, pp. 405-422, Mar. 1968.

[212] Y. Park, M. Diez-Silva, G. Popescu, G. Lykotrafitis, W. Choi, M. S. Feld, and S. Suresh, "Refractive index maps and membrane dynamics of human red blood cells parasitized by Plasmodium falciparum," Proceedings of the National Academy of Sciences, vol. 105, no. 37, pp. 13730-13735, Sep. 2008.

[213] M. Xu and L. V. Wang, "Photoacoustic imaging in biomedicine," Review of Scientific Instruments, vol. 77, no. 4, pp. 041101-041101-22, Apr. 2006.

[214] D.-K. Yao, R. Chen, K. Maslov, Q. Zhou, and L. V. Wang, "Optimal ultraviolet wavelength for in vivo photoacoustic imaging of cell nuclei," Journal of Biomedical Optics, vol. 17, no. 5, pp. 056004-056004-7, May 2012. 
[215] W. Bost, F. Stracke, M. Fournelle, and R. Lemor, "Developing a high-resolution photoacoustic microscopy platform," in 4th European Conference of the International Federation for Medical and Biological Engineering, 2009, pp. 448-451.

[216] E. Evans and Y.-C. Fung, "Improved measurements of the erythrocyte geometry," Microvascular Research, vol. 4, no. 4, pp. 335-347, Oct. 1972.

[217] W. H. Grover, A. K. Bryan, M. Diez-Silva, S. Suresh, J. M. Higgins, and S. R. Manalis, "Measuring single-cell density," Proceedings of the National Academy of Sciences, vol. 108, no. 27, pp. 10992-10996, Jul. 2011.

[218] M. Godin, A. K. Bryan, T. P. Burg, K. Babcock, and S. R. Manalis, "Measuring the mass, density, and size of particles and cells using a suspended microchannel resonator," Applied Physics Letters, vol. 91, no. 12, pp. 123121-123121-3, Sep. 2007.

[219] A. S. Dukhin, P. J. Goetz, and T. G. M. van de Ven, "Ultrasonic characterization of proteins and blood cells," Colloids and Surfaces B: Biointerfaces, vol. 53, no. 2, pp. 121-126, Dec. 2006.

[220] E. T. A. Mohamed, A. E. Kamanyi, M. Pluta, and W. Grill, "Age-dependent acoustic and microelastic properties of red blood cells determined by vector contrast acoustic microscopy," Microscopy and Microanalysis, vol. 18, no. 3, pp. 436-444, Jun. 2012.

[221] P. B. Canham, "The minimum energy of bending as a possible explanation of the biconcave shape of the human red blood cell," Journal of Theoretical Biology, vol. 26, no. 1, pp. 61-81, Jan. 1970.

[222] W. H. Reinhart and S. Chien, "Red cell rheology in stomatocyte-echinocyte transformation: roles of cell geometry and cell shape," Blood, vol. 67, no. 4, pp. 1110-1118, Apr. 1986.

[223] N. S. Gov and S. A. Safran, "Red Blood Cell Membrane Fluctuations and Shape Controlled by ATP-Induced Cytoskeletal Defects," Biophysical Journal, vol. 88, no. 3, pp. 1859-1874, Mar. 2005. 
[224] K. K. Shung, R. A. Sigelmann, and J. M. Reid, "Scattering of Ultrasound by Blood," IEEE Transactions on Biomedical Engineering, vol. BME-23, no. 6, pp. 460-467, Nov. 1976.

[225] Cloutier G. and Z. Qin, "Ultrasound backscattering from non-aggregating and aggregating erythrocytes-a review," Biorheology, vol. 34, no. 6, p. 443, 1997.

[226] I. Fontaine, M. Bertrand, and G. Cloutier, "A System-Based Approach to Modeling the Ultrasound Signal Backscattered by Red Blood Cells," Biophysical Journal, vol. 77, no. 5, pp. 2387-2399, Nov. 1999.

[227] W. T. Coakley, D. W. Bardsley, M. A. Grundy, F. Zamani, and D. J. Clarke, "Cell manipulation in ultrasonic standing wave fields," Journal of Chemical Technology \& Biotechnology, vol. 44, no. 1, pp. 43-62, 1989.

[228] V. Kachel, E. Kordwig, and E. Glossner, "Uniform lateral orientation, caused by flow forces, of flat particles in flow-through systems," Journal of Histochemistry \& Cytochemistry, vol. 25, no. 7, pp. 774-780, Jul. 1977.

[229] J. Dupire, M. Socol, and A. Viallat, "Full dynamics of a red blood cell in shear flow," Proceedings of the National Academy of Sciences, vol. 109, no. 51, pp. 20808-20813, Dec. 2012.

[230] Baddour, Ralph E, High Frequency Ultrasound Scattering from Microspheres and Single Cells. Thesis (M.Sc.), University of Toronto, 2004.

[231] K. A. Gutowski, J. L. Hudson, and D. Aminoff, "Flow cytometric analysis of human erythrocytes: I. Probed with lectins and immunoglobulins," Experimental Gerontology, vol. 26, no. 4, pp. 315-326, 1991.

[232] M. Piagnerelli, K. Z. Boudjeltia, D. Brohee, A. Vereerstraeten, P. Piro, J. Vincent, and M. Vanhaeverbeek, "Assessment of erythrocyte shape by flow cytometry techniques," Journal of Clinical Pathology, vol. 60, no. 5, pp. 549-554, May 2007.

[233] G. Brecher and M. Bessis, "Present Status of Spiculed Red Cells and Their Relationship to the Discocyte-Echinocyte Transformation: A Critical Review," Blood, vol. 40, no. 3, pp. 333-344, Sep. 1972. 
[234] S. Perrotta, P. G. Gallagher, and N. Mohandas, "Hereditary spherocytosis," The Lancet, vol. 372, no. 9647, pp. 1411-1426, 2008.

[235] H. F. Zhang, K. Maslov, M. Sivaramakrishnan, G. Stoica, and L. V. Wang, "Imaging of hemoglobin oxygen saturation variations in single vessels in vivo using photoacoustic microscopy," Applied Physics Letters, vol. 90, no. 5, pp. 053901053901-3, Jan. 2007.

[236] R. K. Saha, S. Karmakar, and M. Roy, "Computational Investigation on the Photoacoustics of Malaria Infected Red Blood Cells," PLoS ONE, vol. 7, no. 12, p. e51774, Dec. 2012.

[237] B. Blasi, A. D’Alessandro, N. Ramundo, and L. Zolla, "Red blood cell storage and cell morphology," Transfusion Medicine, vol. 22, no. 2, pp. 90-96, 2012.

[238] K. L. Scott, J. Lecak, and J. P. Acker, "Biopreservation of Red Blood Cells: Past, Present, and Future," Transfusion Medicine Reviews, vol. 19, no. 2, pp. 127-142, Apr. 2005.

[239] T. P. Butler and P. M. Gullino, "Quantitation of Cell Shedding into Efferent Blood of Mammary Adenocarcinoma," Cancer Research, vol. 35, no. 3, pp. 512-516, Mar. 1975.

[240] L. A. Liotta, J. Kleinerman, and G. M. Saidel, "Quantitative Relationships of Intravascular Tumor Cells, Tumor Vessels, and Pulmonary Metastases following Tumor Implantation,” Cancer Research, vol. 34, no. 5, pp. 997-1004, May 1974.

[241] I. J. Fidler, "The relationship of embolic homogeneity, number, size and viability to the incidence of experimental metastasis," European Journal of Cancer, vol. 9, no. 3, pp. 223-227, Mar. 1973.

[242] E. Racila, D. Euhus, A. J. Weiss, C. Rao, J. McConnell, L. W. M. M. Terstappen, and J. W. Uhr, "Detection and characterization of carcinoma cells in the blood," Proceedings of the National Academy of Sciences, vol. 95, no. 8, pp. 4589-4594, Apr. 1998. 
[243] L. w. m. m. Terstappen, C. Rao, S. Gross, V. Kotelnikov, E. Racilla, J. Uhr, and A. Weiss, "Flowcytometry - Principles and Feasibility in Transfusion Medicine. Enumeration of Epithelial Derived Tumor Cells in Peripheral Blood," Vox Sanguinis, vol. 74, no. S2, pp. 269-274, 1998.

[244] S. J. Simpson, M. Vachula, M. J. Kennedy, H. Kaizer, J. S. Coon, R. Ghalie, S. Williams, and D. Van Epps, "Detection of tumor cells in the bone marrow, peripheral blood, and apheresis products of breast cancer patients using flow cytometry," Experimental Hematology, vol. 23, no. 10, p. 1062, 1995.

[245] R. A. Ghossein and J. Rosai, "Polymerase chain reaction in the detection of micrometastases and circulating tumor cells," Cancer, vol. 78, no. 1, pp. 10-16, 1996.

[246] S.-K. Kraeft, A. Ladanyi, K. Galiger, A. Herlitz, A. C. Sher, D. E. Bergsrud, G. Even, S. Brunelle, L. Harris, R. Salgia, T. Dahl, J. Kesterson, and L. B. Chen, "Reliable and Sensitive Identification of Occult Tumor Cells Using the Improved Rare Event Imaging System," Clinical Cancer Research, vol. 10, no. 9, pp. 3020-3028, May 2004.

[247] H. B. Hsieh, D. Marrinucci, K. Bethel, D. N. Curry, M. Humphrey, R. T. Krivacic, J. Kroener, L. Kroener, A. Ladanyi, N. Lazarus, P. Kuhn, R. H. Bruce, and J. Nieva, "High speed detection of circulating tumor cells," Biosensors and Bioelectronics, vol. 21, no. 10, pp. 1893-1899, Apr. 2006.

[248] V. Zieglschmid, C. Hollmann, and O. Böcher, "Detection of disseminated tumor cells in peripheral blood," Critical Reviews in Clinical Laboratory Sciences, vol. 42, no. 2 , pp. 155-196, 2005.

[249] J. E. Hardingham, D. Kotasek, B. Farmer, R. N. Butler, J.-X. Mi, R. E. Sage, and A. Dobrovic, "Immunobead-PCR: A Technique for the Detection of Circulating Tumor Cells Using Immunomagnetic Beads and the Polymerase Chain Reaction," Cancer Research, vol. 53, no. 15, pp. 3455-3458, Aug. 1993. 
[250] R. M. Weight, P. S. Dale, and J. A. Viator, "Detection of circulating melanoma cells in human blood using photoacoustic flowmetry," in Annual International Conference of the IEEE Engineering in Medicine and Biology Society, 2009, pp. 106-109.

[251] J. Novak, I. Georgakoudi, X. Wei, A. Prossin, and C. P. Lin, "In vivo flow cytometer for real-time detection and quantification of circulating cells," Optics Letters, vol. 29, no. 1, pp. 77-79, Jan. 2004.

[252] W. He, H. Wang, L. C. Hartmann, J.-X. Cheng, and P. S. Low, "In vivo quantitation of rare circulating tumor cells by multiphoton intravital flow cytometry," Proceedings of the National Academy of Sciences, vol. 104, no. 28, pp. 11760-11765, Jul. 2007.

[253] V. P. Zharov, E. I. Galanzha, E. V. Shashkov, N. G. Khlebtsov, and V. V. Tuchin, “In vivo photoacoustic flow cytometry for monitoring of circulating single cancer cells and contrast agents," Optics Letters, vol. 31, no. 24, pp. 3623-3625, Dec. 2006.

[254] E. I. Galanzha, E. V. Shashkov, P. M. Spring, J. Y. Suen, and V. P. Zharov, "In vivo, Noninvasive, Label-Free Detection and Eradication of Circulating Metastatic Melanoma Cells Using Two-Color Photoacoustic Flow Cytometry with a Diode Laser," Cancer Research, vol. 69, no. 20, pp. 7926-7934, Oct. 2009.

[255] E. I. Galanzha, E. V. Shashkov, T. Kelly, J.-W. Kim, L. Yang, and V. P. Zharov, "In vivo magnetic enrichment and multiplex photoacoustic detection of circulating tumour cells," Nature Nanotechnology, vol. 4, no. 12, pp. 855-860, Dec. 2009.

[256] R. M. Weight, J. a Viator, P. S. Dale, C. W. Caldwell, and A. E. Lisle, "Photoacoustic detection of metastatic melanoma cells in the human circulatory system," Optics Letters, vol. 31, no. 20, pp. 2998-3000, Oct. 2006.

[257] C. O’Brien, J. Mosley, B. S. Goldschmidt, and J. A. Viator, "Detection and capture of single circulating melanoma cells using photoacoustic flowmetry," in Proceedings of SPIE, 2010, vol. 7564, p. 75641D-75641D.

[258] G. Gutierrez-Juarez, S. K. Gupta, M. Al-Shaer, L. Polo-Parada, P. S. Dale, C. Papageorgio, and J. a Viator, "Detection of melanoma cells in vitro using an optical 
detector of photoacoustic waves," Lasers in Surgery and Medicine, vol. 42, no. 3, pp. 274-81, Mar. 2010.

[259] D. A. Nedosekin, M. Sarimollaoglu, J. Ye, E. I. Galanzha, and V. P. Zharov, "In vivo ultra-fast photoacoustic flow cytometry of circulating human melanoma cells using near-infrared high-pulse rate lasers," Cytometry Part A, vol. 79A, no. 10, pp. 825833, Oct. 2011.

[260] C. M. O’Brien, K. Rood, S. Sengupta, S. K. Gupta, T. DeSouza, A. Cook, and J. A. Viator, "Detection and Isolation of Circulating Melanoma Cells using Photoacoustic Flowmetry," Journal of Visualized Experiments, no. 57, Nov. 2011.

[261] J. A. Viator, S. Gupta, B. S. Goldschmidt, K. Bhattacharyya, R. Kannan, R. Shukla, P. S. Dale, E. Boote, and K. Katti, "Gold Nanoparticle Mediated Detection of Prostate Cancer Cells Using Photoacoustic Flowmetry with Optical Reflectance," Journal of Biomedical Nanotechnology, vol. 6, no. 2, pp. 187-191, 2010.

[262] D. R. McCormack, K. Bhattacharyya, R. Kannan, K. Katti, and J. a Viator, "Enhanced photoacoustic detection of melanoma cells using gold nanoparticles," Lasers in Surgery and Medicine, vol. 43, no. 4, pp. 333-8, Apr. 2011.

[263] V. P. Zharov, E. I. Galanzha, E. V. Shashkov, J.-W. Kim, N. G. Khlebtsov, and V. V. Tuchin, "Photoacoustic flow cytometry: principle and application for real-time detection of circulating single nanoparticles, pathogens, and contrast dyes in vivo," Journal of Biomedical Optics, vol. 12, no. 5, pp. 051503-051503-14, Oct. 2007.

[264] X. Hu, C.-W. Wei, J. Xia, I. Pelivanov, M. O’Donnell, and X. Gao, “Trapping and Photoacoustic Detection of CTCs at the Single Cell per Milliliter Level with Magneto-Optical Coupled Nanoparticles," Small, vol. 9, no. 12, pp. 2046-2052, 2013.

[265] C.-W. Wei, J. Xia, I. Pelivanov, X. Hu, X. Gao, and M. O'Donnell, “Trapping and dynamic manipulation of polystyrene beads mimicking circulating tumor cells using targeted magnetic/photoacoustic contrast agents," Journal of Biomedical Optics, vol. 17, no. 10, pp. 101517-1, Aug. 2012. 
[266] E. I. Galanzha, E. Shashkov, M. Sarimollaoglu, K. E. Beenken, A. G. Basnakian, M. E. Shirtliff, J.-W. Kim, M. S. Smeltzer, and V. P. Zharov, "In Vivo Magnetic Enrichment, Photoacoustic Diagnosis, and Photothermal Purging of Infected Blood Using Multifunctional Gold and Magnetic Nanoparticles," PLOS ONE, vol. 7, no. 9, p. e45557, Sep. 2012.

[267] X. Qian, X.-H. Peng, D. O. Ansari, Q. Yin-Goen, G. Z. Chen, D. M. Shin, L. Yang, A. N. Young, M. D. Wang, and S. Nie, "In vivo tumor targeting and spectroscopic detection with surface-enhanced Raman nanoparticle tags," Nature Biotechnology, vol. 26, no. 1, pp. 83-90, Jan. 2008.

[268] K. Bhattacharyya, B. S. Goldschmidt, M. Hannink, S. Alexander, and J. A. Viator, "Gold nanoparticle mediated detection of circulating cancer cells," Clinics in Laboratory Medicine, vol. 32, no. 1, pp. 89-101, Mar. 2012.

[269] E. M. Strohm, E. S. L. Berndl, and M. C. Kolios, "A photoacoustic technique to measure the properties of single cells," in Proceedings of SPIE, 2013, p. 85814D85814D-6.

[270] E. M. Strohm and M. C. Kolios, "Sound speed estimation in single cells using the ultrasound backscatter power spectrum," in ICA 2013 Montreal, Montreal, Canada, 2013, vol. 19, p. 075012.

[271] B. J. Bain, Blood Cells: A Practical Guide, 4th ed. Wiley-Blackwell, 2006.

[272] S. Shoji and K. Kawai, "Flow Control Methods and Devices in Micrometer Scale Channels," in Microfluidics, B. Lin, Ed. Springer Berlin Heidelberg, 2011, pp. 1-25. 